\title{
RADIATION CONDITIONS AND INTEGRAL REPRESENTATIONS FOR CLIFFORD ALGEBRA-VALUED NULL SOLUTIONS OF THE ITERATED PERTURBED DIRAC OPERATOR
}

A Dissertation
presented to
the Faculty of the Graduate School
at the University of Missouri-Columbia
In Partial Fulfillment
of the Requirements for the Degree
Doctor of Philosophy
NICHOLAS H. OKAMOTO
Dr. Dorina Mitrea, Dissertation Supervisor
JULY 2017


The undersigned, appointed by the dean of the Graduate School, have examined the dissertation entitled

$$
\begin{aligned}
& \text { Radiation Conditions and Integral Representations } \\
& \text { for Clifford Algebra-Valued Null-Solutions of the } \\
& \text { Iterated Perturbed Dirac Operator }
\end{aligned}
$$

presented by Nicholas Okamoto, a candidate for the degree of doctor of philosophy, and hereby certify that, in their opinion, it is worthy of acceptance.

Professor Dorina Mitrea

Professor Marius Mitrea

Professor Jan Segert

Professor Dorina Kosztin 


\section{ACKNOWLEDGEMENTS}

I would like to express my deepest gratitude to Professor Dorina Mitrea. It has been both an honor and a pleasure to work with her over the past few years. Her invaluable guidance and expertise were indispensable in getting me to where I am today.

Another person I am sincerely thankful to have met is Professor Marius Mitrea. His enthusiasm, charisma, vast knowledge of the field, and the refreshing clarity of his lectures kept me eager to attend every advanced course he would offer.

I am grateful to many other professors at the University of Missouri who have made significant impacts on my development as a mathematician. A few who come to mind most prominently are professors Tanya Christiansen, Stamatis Dostoglou, Adam Helfer, Alexander Koldobsky, Bahram Mashhoon, Stephen Montgomery-Smith, Jan Segert, and Shuguang Wang.

Finally, I want to thank the MU Math Department for partially funding my participation at three Clifford algebra conferences, and for offering me edifying teaching assistantships throughout my graduate career. 


\section{TABLE OF CONTENTS}

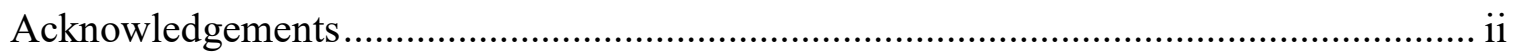

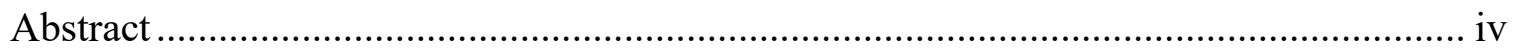

Chapter

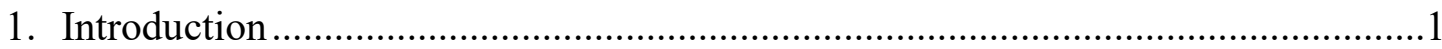

2. Clifford algebras and Dirac Operators .......................................................... 6

3. Clifford-valued distributions and an integration by parts formula........................12

4. The radiating fundamental solution for the Helmholtz operator..........................27

5. Fundamental solutions for iterated Helmholtz operators ....................................33

6. Fundamental solutions for iterated perturbed Dirac operators..............................53

7. Radiation conditions and integral representations for null-solutions of iterated perturbed Dirac operators ........................................................................64

8. Radiation conditions and integral representations for null-solutions of iterated

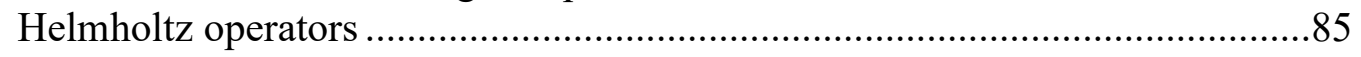

9. A Liouville type theorem for iterated perturbed Dirac operators .........................90 Appendix

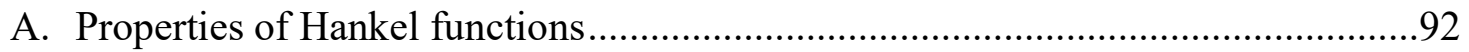

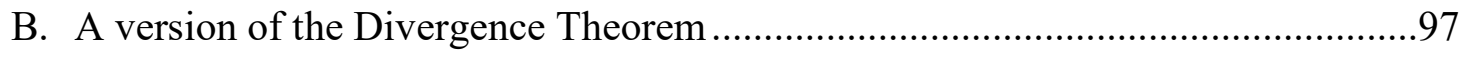

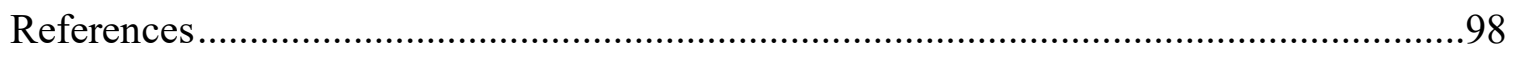

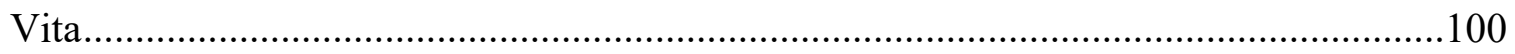




\title{
RADIATION CONDITIONS AND INTEGRAL REPRESENTATIONS FOR CLIFFORD ALGEBRA-VALUED NULL SOLUTIONS OF THE ITERATED PERTURBED DIRAC OPERATOR
}

\author{
Nicholas H. Okamoto \\ Dr. Dorina Mitrea, Dissertation Supervisor
}

\begin{abstract}
In 1912 Arnold Sommerfeld introduced a special decay condition at infinity to address uniqueness issues for certain boundary value problems involving the Helmholtz operator in an exterior domain. Examples of such boundary value problems arise in optical diffraction theory and radio wave propagation. This decay condition, which has become known as Sommerfeld's radiation condition, has been subsequently adapted to various other operators of interest in mathematics, engineering, and physics. Examples include the Silver-Muller radiation condition for the Maxwell system, and radiation conditions for certain perturbed Dirac operators.

In this dissertation, we continue this line of research by considering iterated perturbed Dirac operators. Among other things, suitable radiation conditions are identified which allow us to prove integral representation formulas for Clifford algebravalued null-solutions of iterated perturbed Dirac operators.
\end{abstract}




\section{Introduction}

Given a complex-valued function $u$ which is a null-solution of the Helmholtz operator $\Delta+k^{2}$, with wave number $k \in(0, \infty)$, in the neighborhood of infinity in $\mathbb{R}^{n}$, the celebrated Sommerfeld radiation condition for $u$ reads, in polar coordinates,

$$
\lim _{r \rightarrow \infty}\left\{r^{(n-1) / 2}\left(\frac{\partial}{\partial r}-i k\right) u(r \omega)\right\}=0 \quad \text { uniformly in all directions } \omega \in S^{n-1} .
$$

This radiation condition was originally introduced by A. Sommerfeld in 1912 to address uniqueness issues with regard to certain exterior boundary value problems involving the Helmholtz equation. The examples of boundary value problems that require radiation conditions cited in [21] are from optical diffraction theory and radio wave propagation.

For example, an archetypal scattering problem involves solving the Dirichlet boundary value problem

$$
\left\{\begin{array}{l}
u \in C^{\infty}(\bar{\Omega}) \text { in } \Omega, \\
\left(\Delta+k^{2}\right) u=0 \text { in } \Omega, \\
\left.u\right|_{\partial \Omega}=f \text { on } \partial \Omega,
\end{array}\right.
$$

where $\Omega$ is an exterior domain, with $\partial \Omega$ representing the boundary of the finite rigid body producing the scattering pattern. In early scattering applications, prior to having the concise mathematical criterion in (1.1) to isolate the desired outgoing wave solution, physical considerations needed to be invoked in order to rule out solutions that include any incoming wave components (cf. [20, pp. 388-389]).

From a mathematical point of view, since $\Omega$ is an exterior domain it is natural to think of infinity as being a point on the boundary. As such, dealing with a 
boundary value problem in this setting does require specifying the nature of the behavior of the solution $u$ at this boundary point. For the limiting case $k=0$ of the Helmholtz operator $\Delta+k^{2}$, corresponding to the standard Laplacian in $\mathbb{R}^{n}$, one typically imposes in such a setting the requirement that the function $u$ is harmonic at infinity. The latter condition, which may be expressed as the demand that the Kelvin transform of $u$, i.e., the function $\widetilde{u}(x):=u\left(x /|x|^{2}\right)|x|^{2-n}$, has a removable singularity at the origin. Hence, if $u$ is harmonic at infinity

$$
L:=\lim _{x \rightarrow 0} u\left(x /|x|^{2}\right)|x|^{2-n} \text { exists. }
$$

In turn, this readily implies that if $u$ is harmonic at infinity then $\lim _{x \rightarrow \infty} u(x)=L$ if $n=2$ and $\lim _{x \rightarrow \infty} u(x)=0$ if $n \geq 3$. This observation leads to a characterization of harmonicity at infinity purely in terms of the size of the function $u$. These considerations, pertaining to the Laplacian $\Delta$, are in sharp contrast with the case of the Helmholtz operator $\Delta+k^{2}$ with $k \in(0, \infty)$ since, as is apparent from (1.1), it is now an algebraic expression involving $u$ and partial derivatives of $u$, linked with coefficients depending on the wave number $k$, which is required to decay at infinity.

Historically, the Sommerfeld radiation condition (1.1) was also accompanied by the so-called Sommerfeld finiteness condition

$$
u(x)=O\left(|x|^{-(n-1) / 2}\right) \text { as }|x| \rightarrow \infty .
$$

However, in 1956, C.H. Wilcox demonstrated that (1.4) is implied by (1.1), rendering the finiteness condition superfluous.

Over the years, the issue of determining radiations conditions for various classes 
of operators has received considerable attention, as evidenced by the work in [22], [23], [24], [27], and [28], to cite a few.

In more recent developments, E. Marmolejo-Olea, D. Mitrea, I. Mitrea, and M. Mitrea have developed (in [9]) a unified approach to radiation conditions for the entire class of null-solutions of the Helmholtz operator which are Clifford algebravalued. They provided a multitude of novel radiation conditions which naturally contain the Sommerfeld and Silver-Müller radiation conditions in the case of nullsolutions for the scalar Helmholtz operator and the Maxwell system, respectively, which also encompass as a particular case the radiation condition introduced by McIntosh and Mitrea (in [11]) for perturbed Dirac operators.

The direction we take in this thesis is to continue up the ladder of higher powers of the perturbed Dirac operator. Seeking integral representation formulas for Clifford algebra-valued null-solutions of iterated perturbed Dirac operators, we construct fundamental solutions and develop appropriate radiation conditions along the way. Results in [9] provide a useful touchstone with which to compare the first and second iterations of our larger family of results.

We wish to emphasize that the perturbed Dirac operators alluded to above are first-order differential operators which may be used to factor out the (second-order) Helmholtz operator. In particular, the family of iterated powers of such perturbed Dirac operators contains iterated powers of the Helmholtz operator $\Delta+k^{2}$.

The layout of the thesis is as follows. Chapter 2 provides a very brief introduction to the basic objects and operators of Clifford analysis utilized in this thesis, while chapter 3 explores these ideas in the context of distribution theory. Here we will also prove an integration by parts formula (cf. Theorem 3.3) involving Clifford algebra-valued distributions on a domain with compact Lipschitz boundary. Its proof relies on a particular case of the sharp Divergence Theorem (see the Appendix 
section B) proved in [13].

For ease of reference, in chapter 4 we collect some results from [9] which are relevant to our work and which motivated our present investigative trajectory. Having the fundamental solution for the Helmholtz operator available here provides us with a foundational rung for the ladder of fundamental solutions we develop in the following two chapters. As such, this is also the paper on which our notational choices are based.

In chapters $5 \& 6$ we develop fundamental solutions for iterated Helmholtz and iterated perturbed Dirac operators respectively, using properties of Hankel functions (see the Appendix section A) to explore both their singular behavior near the origin and their limiting behavior as the argument tends to infinity. Knowledge of their behavior approaching the origin is used while demonstrating they are fundamental solutions, while knowledge of their behavior towards infinity is crucial for determining the appropriate radiation conditions needed for integral representations developed in chapter 7. The fundamental solutions of the iterated Helmholtz operators were found by constructing (through adaptive trial-and-error) functions with the desired recursive properties proven at the beginning of chapter 5 . In chapter 6, the fundamental solutions developed in the previous chapter provided us with the fundamental solutions for the even iterations of the perturbed Dirac operator, and the formulas for the odd cases follow from applying the perturbed Dirac operator to the formulas for the even cases.

Chapter 7 is where we arrive at our main results. With the fundamental solutions of the previous chapters at our disposal, obtaining integral representations for Clifford algebra-valued null-solutions of iterated perturbed Dirac operators on bounded Lipschitz domains is a direct consequence of repeated applications of the integration by parts formula derived in chapter 3 . The more challenging task is to 
extend this result to exterior Lipschitz domains. Attaining this goal involved the development of precise radiation conditions.

The final two chapters before the Appendix identify important consequences of our main result from Chapter 7. In Chapter 8 we consider even powers of the perturbed Dirac operator to provide radiation conditions and integral representations for null-solutions of iterated Helmholtz operators. Chapter 9 provides a Liouville type theorem for iterated perturbed Dirac operators.

A note on notation; throughout this paper fix $n \in \mathbb{N}, n \geq 2$, and set

$$
\widehat{x}:=x /|x| \text { for } \quad x \in \mathbb{R}^{n} \backslash\{0\} .
$$

Call $\Omega \subseteq \mathbb{R}^{n}$ an exterior domain if its complement $\mathbb{R}^{n} \backslash \Omega$ is a compact set. A Lipschitz domain $\Omega$, i.e. a domain with Lipschitz boundary $\partial \Omega$, is a domain whose boundary may be represented locally as the graph of a Lipschitz continuous function. The $n$-dimensional Lebesgue measure in $\mathbb{R}^{n}$ will be denoted by $\mathscr{L}^{n}$, while the $(n-1)$-dimensional Hausdorff measure in $\mathbb{R}^{n}$ will be denoted by $\mathscr{H}^{n-1}$. 


\section{Clifford algebras and Dirac operators}

Since Clifford algebras provide the mathematical grammar employed throughout this paper, our first order of business will be to introduce some basic features and properties of Clifford calculus. For more background material and further general references on Clifford algebras and related matters, the interested reader is referred to the monographs [3], [7], and [14].

Given some number $m \in \mathbb{N}_{0}$, the (complex) Clifford algebra $\left(\mathrm{Cl}_{m},+, \odot\right)$ is the minimal enlargement of $\mathbb{C}^{m}$ to a unitary complex algebra, which is not generated (as an algebra) by any proper subspace of $\mathbb{C}^{m}$, and such that

$$
x \odot x=-|x|^{2} \quad \text { for any } x \in \mathbb{R}^{m} \hookrightarrow \mathbb{C}^{m} \hookrightarrow C \ell_{m} .
$$

This identity readily implies that, if $\left\{e_{j}\right\}_{1 \leq j \leq m}$ is the standard orthonormal basis in $\mathbb{R}^{m}$, then

$$
e_{j} \odot e_{j}=-1 \quad \text { and } \quad e_{j} \odot e_{k}=-e_{k} \odot e_{j} \text { whenever } 1 \leq j \neq k \leq m \text {. }
$$

In particular, identifying the canonical basis $\left\{e_{j}\right\}_{1 \leq j \leq m}$ from $\mathbb{R}^{m}$ with the $m$ imaginary units generating $C \ell_{m}$, yields the embedding

$$
\mathbb{C}^{m} \hookrightarrow C \ell_{m}, \quad \mathbb{C}^{m} \ni x=\left(x_{1}, \ldots, x_{m}\right) \equiv \sum_{j=1}^{m} x_{j} e_{j} \in C \ell_{m} .
$$

Any element $u \in C \ell_{m}$ can be uniquely represented in the form

$$
u=\sum_{I} u_{I} e_{I}=\sum_{\ell=0}^{m} \sum_{|I|=\ell}^{\prime} u_{I} e_{I}, \quad u_{I} \in \mathbb{C} .
$$


Here $e_{I}$ stands for the product $e_{i_{1}} \odot e_{i_{2}} \odot \cdots \odot e_{i_{\ell}}$ if $I=\left(i_{1}, i_{2}, \ldots, i_{\ell}\right)$ and $e_{0}:=e_{\emptyset}:=1$ is the multiplicative unit. Also, $\Sigma^{\prime}$ indicates that the sum is performed only over strictly increasing multi-indices, i.e., $l$-tuples $I=\left(i_{1}, i_{2}, \ldots, i_{\ell}\right)$ with $1 \leq i_{1}<i_{2}<$ $\cdots<i_{\ell} \leq m$. Subsequently, we will work with Clifford algebra-valued functions $u$ as in (2.4) which have their coefficients $u_{I}$ belonging to a certain Banach space. Specifically, given an open set $\Omega \subseteq \mathbb{R}^{n}$, we will work with $u$ as in (2.4) with the additional property that all $u_{I}^{\prime}$ s belong to $\mathscr{C}^{1}(\Omega)$, in which case we will write $u \in \mathscr{C}^{1}\left(\Omega, C \ell_{m}\right)$. Similarlly, we will use the notation $u \in \mathscr{C}^{0}\left(\bar{\Omega}, C \ell_{m}\right)$, or $u \in L^{1}\left(\Omega, C \ell_{m}\right)$, or $u \in L_{\text {loc }}^{1}\left(\Omega, C \ell_{m}\right)$, etc., whenever the coefficients $u_{I}$ are continuous on the closure of $\Omega$, or are absolutely integrable on $\Omega$, or are absolutely integrable on compact subsets of $\Omega$, etc.

We endow $C \ell_{m}$ with the natural Hilbert space structure

$$
\langle u, v\rangle:=\sum_{I} u_{I} v_{I}, \quad \text { if } u=\sum_{I} u_{I} e_{I}, v=\sum_{I} v_{I} e_{I} \in C \ell_{m} .
$$

If we now define a complex conjugation on $C \ell_{m}$ by setting $u^{c}:=\sum_{I} u_{I}^{c} e_{I}$ for each $u=\sum_{I} u_{I} e_{I}$, where $z^{c}$ stands for the usual complex conjugation of $z \in \mathbb{C}$, the given Hilbert space structure (2.5) further induces the natural Euclidean metric

$$
|u|=\sqrt{\left\langle u, u^{c}\right\rangle}=\left\{\sum_{I}\left|u_{I}\right|^{2}\right\}^{1 / 2} \text { for each } u=\sum_{I} u_{I} e_{I} \in C \ell_{m}
$$

which satisfies the usual triangle inequality

$$
|u+v| \leq|u|+|v|, \quad \forall u, v \in C \ell_{m} .
$$

The Clifford conjugation on $C \ell_{m}$, denoted by 'bar', is defined as the unique real- 
linear involution on $C \ell_{m}$ for which $\overline{e_{I}} \odot e_{I}=e_{I} \odot \overline{e_{I}}=1$ for any multi-index $I$. More specifically, given $u=\sum_{I} u_{I} e_{I} \in C \ell_{m}$ we set $\bar{u}:=\sum_{I} u_{I} \overline{e_{I}}$ where, for each $I=\left(i_{1}, i_{2}, \ldots, i_{\ell}\right)$ with $1 \leq i_{1}<i_{2}<\cdots<i_{\ell} \leq m$,

$$
\overline{e_{I}}=(-1)^{\ell} e_{i_{\ell}} \odot e_{i_{\ell-1}} \odot \cdots \odot e_{i_{1}} .
$$

Thus, in particular,

$$
\overline{e_{j}}=-e_{j}, \quad \forall j \in\{1, \ldots, m\},
$$

which further implies that

$$
\bar{x}=-x \text { for each } x \in \mathbb{C}^{m} \hookrightarrow C \ell_{m} .
$$

One can also verify without difficulty that

$$
\overline{\bar{u}}=u, \quad|\bar{u}|=|u|, \text { and } \overline{u \odot v}=\bar{v} \odot \bar{u}, \quad \forall u, v \in C \ell_{m} \text {. }
$$

The complex conjugation on $C \ell_{m}$ is defined as $u^{c}:=\sum_{I} u_{I}^{c} e_{I}$ for each $u=\sum_{I} u_{I} e_{I}$, where $a^{c}$ denotes the usual complex conjugation of $a \in \mathbb{C}$. Let us also define the scalar part of $u=\sum_{I} u_{I} e_{I} \in C \ell_{m}$ as $u_{0}:=u_{\emptyset}$.

Other basic properties, applicable to arbitrary $u, v \in C \ell_{m}$, are as follows:

$$
\begin{gathered}
|u|^{2}=\left(u \odot \bar{u}^{c}\right)_{0}=\left(\bar{u}^{c} \odot u\right)_{0}=\left\langle u, u^{c}\right\rangle, \\
\langle u, v\rangle=(u \odot \bar{v})_{0}=(\bar{u} \odot v)_{0}, \\
u_{0}=(\bar{u})_{0} \\
(u \odot v)_{0}=(v \odot u)_{0}
\end{gathered}
$$




$$
(u \odot v)^{c}=\left(u^{c}\right) \odot\left(v^{c}\right),
$$

and

$$
|u \odot v|=|u \| v| \text { if either } u \text { or } v \text { belongs to } \mathbb{C}^{m} \hookrightarrow C \ell_{m} \text {. }
$$

To see why (2.17) is true, suppose $u \in \mathbb{C}^{m}$. Then, using (2.12), (2.15), (2.11), (2.10), we may write

$$
\begin{aligned}
|u \odot v|^{2} & =\left(u \odot v \odot \overline{u \odot v}^{c}\right)_{0}=\left(\overline{u \odot v}^{c} \odot u \odot v\right)_{0} \\
& =\left(\bar{v}^{c} \odot \bar{u}^{c} \odot u \odot v\right)_{0}=-\left(\bar{v}^{c} \odot u^{c} \odot u \odot v\right)_{0}=|u|^{2}\left(\bar{v}^{c} \odot v\right)_{0} \\
& =|u|^{2}|v|^{2} .
\end{aligned}
$$

The case $v \in \mathbb{C}^{m}$ is treated similarly, this time without using (2.15).

Lemma 2.1. For any $u, v \in C \ell_{m}$ one has

$$
|u+v|^{2}=|u|^{2}+|v|^{2}+2 \operatorname{Re}\left[\left(u \odot \bar{v}^{c}\right)_{0}\right]
$$

Proof.

$$
\begin{aligned}
|u+v|^{2} & =\left[(u+v) \odot \overline{(u+v)}^{c}\right]_{0}=\left[(u+v) \odot\left(\bar{u}^{c}+\bar{v}^{c}\right)\right]_{0} \\
& =\left(u \odot \bar{u}^{c}+v \odot \bar{v}^{c}+u \odot \bar{v}^{c}+v \odot \bar{u}^{c}\right)_{0} \\
& =|u|^{2}+|v|^{2}+\left(u \odot \bar{v}^{c}\right)_{0}+\left(v \odot \bar{u}^{c}\right)_{0}
\end{aligned}
$$

Since by (2.14), (2.11), and (2.16) we have

$$
\left(v \odot \bar{u}^{c}\right)_{0}=\left(u^{c} \odot \bar{v}\right)_{0}=\left[\left(u \odot \bar{v}^{c}\right)_{0}\right]^{c},
$$


identity (2.19) now follows from (2.20) and (2.21).

When simultaneously dealing with two Clifford algebras, say $C \ell_{m_{1}}$ and $C \ell_{m_{2}}$, we canonically view them as the subalgebras of $C \ell_{m}$ where $m:=\max \left\{m_{1}, m_{2}\right\}$ freely generated by $\left\{e_{1}, \ldots, e_{m_{1}}\right\}$ and $\left\{e_{1}, \ldots, e_{m_{2}}\right\}$ respectively. Here is a concrete case of interest where this convention is called for. Let $\Omega$ be an open set in $\mathbb{R}^{n}$. Then the classical (homogeneous) Dirac operator associated with $\mathbb{R}^{n}$ is given by

$$
D:=\sum_{j=1}^{n} e_{j} \odot \partial_{j}
$$

This acts on a function $u \in \mathscr{C}^{1}\left(\Omega, C \ell_{m}\right)$ where $m \in \mathbb{N}_{0}$ according to

$$
D u:=\sum_{j=1}^{n} e_{j} \odot\left(\partial_{j} u\right)
$$

with the right-hand side of (2.23) regarded as a $C \ell_{M^{-}}$valued function, where we set $M:=\max \{n, m\}$. We shall also work with the perturbed Dirac operator

$$
D_{k}:=D+k e_{n+1}
$$

for some complex number $k \in \mathbb{C}$. Hence, given $m \in \mathbb{N}_{0}$ and an arbitrary $u \in$ $\mathscr{C}^{1}\left(\Omega, C \ell_{m}\right)$, we have

$$
D_{k} u=\sum_{j=1}^{n} e_{j} \odot \partial_{j} u+k e_{n+1} \odot u,
$$

with the right-hand side of (2.25) regarded as a $C l_{M^{-}}$-valued function in $\Omega$, for $M:=\max \{n+1, m\}$. Call $u$ monogenic in $\Omega$ if $D u=0$ in $\Omega$, and call $u k$-monogenic in $\Omega$ if $D_{k} u=0$ in $\Omega$.

When the Dirac operator $D$ and the perturbed Dirac operator $D_{k}$ are acting from 
the right on some $u \in \mathscr{C}^{1}\left(\Omega, C \ell_{m}\right)$ we write $u D$ and $u D_{k}$, respectively. Hence, in this scenario,

$$
u D=\sum_{j=1}^{n} \partial_{j} u \odot e_{j} \quad \text { and } \quad u D_{k}=\sum_{j=1}^{n} \partial_{j} u \odot e_{j}+k u \odot e_{n+1} .
$$

One of the basic properties of the Dirac operators introduced above is that they can be thought of as square-roots of familiar second-order differential operators. More precisely, $D$ and $D_{k}$ satisfy

$$
D^{2}=-\Delta \quad \text { and } \quad D_{k}^{2}=-\left(\Delta+k^{2}\right),
$$

where $\Delta:=\sum_{j=1}^{n} \partial_{j}^{2}$ is the usual Laplace operator in $\mathbb{R}^{n}$. 


\section{Clifford-valued distributions and an integration by parts formula}

As usual, given an open nonempty set $\Omega \subseteq \mathbb{R}^{n}$, by $\mathcal{D}^{\prime}(\Omega)$ we shall denote the space of distributions in $\Omega$ and by $\mathcal{D}(\Omega)$ the space of test functions in $\Omega$. In the sequel, we will work with $C \ell_{m}$-valued distributions in $\Omega$, the collection of which will be denoted by $\mathcal{D}^{\prime}\left(\Omega, C \ell_{m}\right)$. More specifically,

$$
\mathcal{D}^{\prime}\left(\Omega, C \ell_{m}\right):=\left\{u=\sum_{\ell=0}^{m} \sum_{|I|=\ell}^{\prime} u_{I} e_{I}: u_{I} \in \mathcal{D}^{\prime}(\Omega)\right\}
$$

These objects obey natural rules, much as ordinary distributions, with the added bonus that the Clifford algebra formalism is in full effect.

The pairing between a $C \ell_{m}$-valued distribution $u=\sum_{\ell=0}^{m} \sum_{|I|=\ell}^{\prime} u_{I} e_{I}$ and a test function $\varphi \in \mathscr{C}_{0}^{\infty}(\Omega)$ is defined by

$$
\langle u, \varphi\rangle\rangle:=\sum_{\ell=0}^{m} \sum_{\mid[\mid=\ell}^{\prime}{ }_{\mathcal{D}^{\prime}(\Omega)}\left\langle u_{I}, \varphi\right\rangle_{\mathcal{D}(\Omega)} e_{I}
$$

where $\mathcal{D}^{\prime}(\Omega)\langle\cdot, \cdot\rangle_{\mathcal{D}(\Omega)}$ denotes the duality pairing between distributions in $\Omega$ and test funnctions in $\Omega$. In particular, it is immediate that the Dirac distribution $\delta=\delta e_{\emptyset}$ satisfies

$$
\langle\langle\delta, \varphi\rangle\rangle={ }_{\mathcal{D}^{\prime}(\Omega)}\langle\delta, \varphi\rangle_{\mathcal{D}(\Omega)} e_{\emptyset}=\varphi(0), \quad \forall \varphi \in \mathcal{D}(\Omega)
$$

To discuss the action of $C \ell_{m}$-differential operators on Clifford algebra-valued distribution, fix some $M \in \mathbb{N}$ arbitrary. Then by an $M^{\text {th }}$-order $C \ell_{m}$-differential 
operator we understand an operator of the form

$$
P:=\sum_{\substack{\alpha \in \mathbb{N}_{0}^{n} \\|\alpha| \leq M}} a_{\alpha} \odot \partial^{\alpha}, \quad \text { where } a_{\alpha} \in C \ell_{m}, \quad \forall \alpha \in \mathbb{N}_{0}^{n}, \quad|\alpha| \leq M
$$

The action of such and operator $P$ on a distribution $u \in \mathcal{D}^{\prime}\left(\Omega, C \ell_{m}\right)$ of the form $u=\sum_{\ell=0}^{m} \sum_{|I|=\ell}^{\prime} u_{I} e_{I}$ with $u_{I} \in \mathcal{D}^{\prime}(\Omega)$ is defined by

$$
P u:=\sum_{\ell=0}^{m} \sum_{|I|=\ell}^{\prime} \sum_{\substack{\alpha \in \mathbb{N}_{0}^{n} \\|\alpha| \leq M}}\left(\partial^{\alpha} u_{I}\right) a_{\alpha} \odot e_{I} \quad \text { in } \mathcal{D}^{\prime}\left(\Omega, C \ell_{m}\right) .
$$

Of course, if $u \in \mathscr{C}^{M}\left(\Omega, C \ell_{m}\right)$, then the $\partial^{\alpha} u_{I}$ are taken point-wise in $\Omega$. Call $u \in$ $\mathcal{D}^{\prime}\left(\mathbb{R}^{n}, C \ell_{m}\right)$ a fundamental solution for some operator $P$ as in (3.4) provided $P u=\delta$ in $\mathcal{D}^{\prime}\left(\mathbb{R}^{n}, C \ell_{m}\right)$.

Our next result shows that under certain growth restrictions near the origin, the point-wise action of a first order differential operator on a locally integrable function in $\mathbb{R}^{n} \backslash\{0\}$ coincides with the action of the respective operator in the distributional sense in $\mathbb{R}^{n} \backslash\{0\}$.

Proposition 3.1. Let $P$ be a $1^{\text {st }}$-order $C \ell_{m}$-differential operator and suppose $u \in \mathscr{C}^{1}\left(\mathbb{R}^{n} \backslash\right.$ $\left.\{0\}, C \ell_{m}\right)$ is such that

$$
|u(x)|=o\left(|x|^{1-n}\right) \text { and }|(D u)(x)|=O\left(|x|^{1-n}\right) \text { as } x \rightarrow 0
$$

In addition, assume that there exists some $v \in L_{l o c}^{1}\left(\mathbb{R}^{n}, C l_{m}\right)$ satisfying $P u=v$ point-wise almost everywhere in $\mathbb{R}^{n}$.

Then $P u=v$ in $\mathcal{D}^{\prime}\left(\mathbb{R}^{n}, C \ell_{m}\right)$. 
Proof. Fix $P, u$, and $v$ as in the hypotheses of the proposition. Then

$$
P=\sum_{j=1}^{n} a_{j} \odot \partial_{j}+b, \quad a_{1}, \ldots, a_{n}, b \in C \ell_{m},
$$

we have

$$
\begin{aligned}
u & =\sum_{\ell=0}^{m} \sum_{|I|=\ell}^{\prime} u_{I} e_{I} \text { with } u_{I} \in \mathscr{C}^{1}\left(\mathbb{R}^{n} \backslash\{0\}\right) \text { satisfying } \\
\left|u_{I}(x)\right| & =o\left(|x|^{1-n}\right) \text { and }\left|\left(\partial_{j} u_{I}\right)(x)\right|=O\left(|x|^{1-n}\right), \quad \forall j \in\{1, \ldots, n\}, \text { as } x \rightarrow 0,
\end{aligned}
$$

and $v \in L_{l o c}^{1}\left(\mathbb{R}^{n}, C l_{m}\right)$ satisfies

$$
v(x)=\sum_{\ell=0}^{m} \sum_{|I|=\ell}^{\prime}\left(\sum_{j=1}^{n}\left(\partial_{j} u_{I}\right)(x) a_{j} \odot e_{I}+u_{I}(x) b \odot e_{I}\right) \quad \text { for a.e. } x \in \mathbb{R}^{n} \text {. }
$$

Fix a test function $\varphi \in \mathscr{C}_{0}^{\infty}\left(\mathbb{R}^{n}\right)$. Making use of (3.5), (3.2), and the definition of distributional derivatives in $\mathcal{D}^{\prime}(\Omega)$ we may write

$$
\begin{aligned}
\langle\langle\mathcal{P}, \varphi\rangle\rangle & =\left\langle\left\langle\sum_{\ell=0}^{m} \sum_{|I|=\ell}^{\prime}\left(\sum_{j=1}^{n}\left(\partial_{j} u_{I}\right) a_{j} \odot e_{I}+u_{I} b \odot e_{I}\right), \varphi\right\rangle\right) \\
& =\sum_{\ell=0}^{m} \sum_{|I|=\ell}^{\prime}\left(\sum_{j=1}^{n}{ }_{\mathcal{D}^{\prime}(\Omega)}\left\langle\partial_{j} u_{I}, \varphi\right\rangle_{\mathcal{D}(\Omega)} a_{j} \odot e_{I}+{ }_{\mathcal{D}^{\prime}(\Omega)}\left\langle u_{I}, \varphi\right\rangle_{\mathcal{D}(\Omega)} b \odot e_{I}\right) \\
& =\sum_{\ell=0}^{m} \sum_{|I|=\ell}^{\prime}\left(-\sum_{j=1}^{n}{ }_{\mathcal{D}^{\prime}(\Omega)}\left\langle u_{I}, \partial_{j} \varphi\right\rangle_{\mathcal{D}(\Omega)} a_{j} \odot e_{I}+{ }_{\mathcal{D}^{\prime}(\Omega)}\left\langle u_{I}, \varphi\right\rangle_{\mathcal{D}(\Omega)} b \odot e_{I}\right) .
\end{aligned}
$$

Choose $R \in(0, \infty)$ sufficiently large so that $\operatorname{supp}(\varphi) \subseteq B(0, R)$. Fix a multi-index $I$ and $j \in\{1, \ldots, n\}$. Note that the assumptions on $u$ ensure that $u_{I}$ is locally integrable in $\mathbb{R}^{n}$. The latter combined with Lebesgue's Dominated Convergence Theorem and 
integration by parts (bearing in mind the support condition on $\varphi$ ) yield

$$
\begin{aligned}
& { }_{\mathcal{D}^{\prime}(\Omega)}\left\langle u_{I}, \partial_{j} \varphi\right\rangle_{\mathcal{D}(\Omega)}=\int_{B(0, R)} u_{I}(x)\left(\partial_{j} \varphi\right)(x) d \mathscr{L}^{n}(x) \\
& =\lim _{\varepsilon \rightarrow 0^{+}} \int_{B(0, R) \backslash B(0, \varepsilon)} u_{I}(x)\left(\partial_{j} \varphi\right)(x) d \mathscr{L}^{n}(x) \\
& =\lim _{\varepsilon \rightarrow 0^{+}}\left[\int_{\partial B(0, \varepsilon)} \frac{-x_{j}}{\varepsilon} u_{I}(x) \varphi(x) d \mathscr{H}^{n-1}(x)\right. \\
& \left.-\int_{B(0, R) \backslash B(0, \varepsilon)}\left(\partial_{j} u_{I}\right)(x) \varphi(x) d \mathscr{L}^{n}(x)\right] .
\end{aligned}
$$

With

$$
\omega_{n-1}:=2 \pi^{n / 2} / \Gamma(n / 2)
$$

representing the area of the unit sphere $S^{n-1}$ in $\mathbb{R}^{n}$ (here and elsewhere, $\Gamma$ stands for the Gamma function), the boundary integral may be further estimated by

$$
\begin{aligned}
\mid \int_{\partial B(0, \varepsilon)} \frac{-x_{j}}{\varepsilon} & u_{I}(x) \varphi(x) d \mathscr{H}^{n-1}(x) \mid \\
& \leq\|\varphi(x)\|_{L^{\infty}\left(\mathbb{R}^{n}\right)} \int_{\partial B(0, \varepsilon)}\left|u_{I}(x)\right| d \mathscr{H}^{n-1}(x) \\
& \leq\|\varphi(x)\|_{L^{\infty}\left(\mathbb{R}^{n}\right)} \omega_{n-1} \varepsilon^{n-1} \max _{x \in \partial B(0, \varepsilon)}|u(x)| \rightarrow 0 \text { as } \varepsilon \rightarrow 0^{+}
\end{aligned}
$$

since $\left|\frac{x_{j}}{\varepsilon}\right| \leq 1$ on $\partial B(0, \varepsilon)$ and $\max _{x \in \partial B(0, \varepsilon)}\{|u(x)|\}=o\left(\varepsilon^{1-n}\right)$ as $\varepsilon \rightarrow 0^{+}$by (3.9). A combination of (3.12)-(3.14) and another application of Lebesgue's Dominated Convergence 
Theorem (note that $\partial_{j} u_{I}$ is a locally integrable function in $\mathbb{R}^{n}$ ) imply

$$
\begin{aligned}
& \mathcal{D}^{\prime}(\Omega)\left\langle u_{I}, \partial_{j} \varphi\right\rangle_{\mathcal{D}(\Omega)}=-\lim _{\varepsilon \rightarrow 0^{+}} \int_{B(0, R) \backslash B(0, \varepsilon)}\left(\partial_{j} u_{I}\right)(x) \varphi(x) d \mathscr{L}^{n}(x) \\
&=-\int_{B(0, R)}\left(\partial_{j} u_{I}\right)(x) \varphi(x) d \mathscr{L}^{n}(x) \\
&=-\mathcal{D}^{\prime}(\Omega) \\
&\left\langle\left(\partial_{j} u_{I}\right), \varphi\right\rangle_{\mathcal{D}(\Omega)},
\end{aligned}
$$

where $\partial_{j} u_{I}$ in the last term in (3.15) is the function obtained after taking the $j$-th point-wise partial derivative of $u_{I}$ in $\mathbb{R}^{n} \backslash\{0\}$.

Since $I, j$ were arbitrary, we may return with (3.15) to (3.11) to write

$$
\begin{aligned}
\langle P u, \varphi\rangle\rangle, & =\sum_{\ell=0}^{m} \sum_{\mid[\mid=\ell}^{\prime}\left(\sum_{j=1}^{n}{ }_{\mathcal{D}^{\prime}(\Omega)}\left\langle\left(\partial_{j} u_{I}\right), \varphi\right\rangle_{\mathcal{D}(\Omega)} a_{j} \odot e_{I}+{ }_{\mathcal{D}^{\prime}(\Omega)}\left\langle u_{I}, \varphi\right\rangle_{\mathcal{D}(\Omega)} b \odot e_{I}\right) \\
& =\langle\langle v, \varphi\rangle\rangle,
\end{aligned}
$$

where the $2^{\text {nd }}$ equality follows from (3.10) and definition (3.2). Since $\varphi \in \mathcal{D}\left(\mathbb{R}^{n}\right)$ is arbitrary, (3.16) implies $P u=v$ in $\mathcal{D}^{\prime}\left(\mathbb{R}^{n}, C \ell_{m}\right)$.

Preparing to introduce an integration by parts formula involving perturbed Dirac operators, we first define the mollifiers $\theta_{\varepsilon}$ below and prove the subsequent lemma. Let $\theta$ satisfy (cf. e.g. [12, Lemma 13.24])

$$
\theta \in \mathscr{C}^{\infty}(\Omega), \quad \theta \geq 0, \quad \operatorname{supp}(\theta) \subseteq B(0,1), \quad \text { and } \quad \int_{\mathbb{R}^{n}} \theta(x) d \mathscr{L}^{n}(x)=1
$$

For $\varepsilon>0$ define

$$
\theta_{\varepsilon}:=\varepsilon^{-n} \theta(x / \varepsilon), \quad \forall x \in \mathbb{R}^{n} .
$$


Consequently,

$$
\begin{gathered}
\theta_{\varepsilon} \in \mathscr{C}^{\infty}(\Omega), \quad \theta_{\varepsilon} \geq 0, \quad \operatorname{supp}\left(\theta_{\varepsilon}\right) \subseteq B(0, \varepsilon), \\
\text { and } \int_{\mathbb{R}^{n}} \theta_{\varepsilon}(x) d \mathscr{L}^{n}(x)=1 .
\end{gathered}
$$

Next, fix an open set $\Omega \subseteq \mathbb{R}^{n}$. For each $f \in L_{\text {loc }}^{1}(\Omega)$ and each $\varepsilon>0$ define

$$
f_{\varepsilon}(x):=\int_{\Omega} \theta_{\varepsilon}(x-y) f(y) d \mathscr{L}^{n}(y) \text { for each } x \in \Omega_{\varepsilon},
$$

where

$$
\Omega_{\varepsilon}:=\{x \in \Omega: \operatorname{dist}(x, \partial \Omega)>\varepsilon\} .
$$

Note that for each $x \in \Omega_{\varepsilon}$ and each $y \in B(x, \varepsilon)$ we have $x-y \in \Omega$. Based on this, the fact that $\operatorname{supp}\left(\theta_{\varepsilon}\right) \subseteq B(0, \varepsilon)$ and that $f$ is locally integrable in $\Omega$, it follows that the integral in (3.20) is absolutely integrable for each $x \in \Omega_{\varepsilon}$, thus $f_{\varepsilon}$ is well-defined. In addition, $f_{\varepsilon} \in C^{\infty}\left(\Omega_{\varepsilon}\right)$ for each $\varepsilon>0$ and we also have

$$
f_{\varepsilon}(x):=\int_{B(0, \varepsilon)} f(x-y) \theta_{\varepsilon}(y) d \mathscr{L}^{n}(y) \quad \text { for each } x \in \Omega_{\varepsilon} .
$$

Some additional properties of the sequence $\left\{f_{\varepsilon}\right\}_{\varepsilon}$ are singled out in the following lemma. Before stating it we also introduce one more notation. If $K \subseteq \Omega$ is a compact set, define

$$
\varepsilon_{K}:=\operatorname{dist}(K, \partial \Omega)
$$

and

$$
K_{\varepsilon}:=\{x \in \Omega: \operatorname{dist}(x, K) \leq \varepsilon\} .
$$

Lemma 3.2. Let $\Omega \in \mathbb{R}^{n}$ be open and $f \in L_{\text {loc }}^{1}(\Omega)$. For each $\varepsilon>0$, recall $\theta_{\varepsilon}$ from (3.18) 
and $f_{\varepsilon}$ from (3.20). Then the following are true.

(1) $f_{\varepsilon} \stackrel{\varepsilon \rightarrow 0^{+}}{\longrightarrow}$ f for $\mathscr{L}^{n}$-a.e. in $\Omega$.

(2) For every compact set $K \subseteq \Omega$ we have $\left\|f_{\varepsilon}\right\|_{L^{1}(K)} \leq\|f\|_{L^{1}\left(K_{\varepsilon}\right)}$ for each $\varepsilon \in\left(0, \varepsilon_{K}\right)$.

(3) For every compact set $K \subseteq \Omega$ we have

$$
f_{\varepsilon} \stackrel{\varepsilon \rightarrow 0^{+}}{\longrightarrow} f \quad \text { in } \quad L^{1}(K)
$$

Proof. Recall that by Lebesgue's Differentiation Theorem, we have that

$$
\lim _{\varepsilon \rightarrow 0^{+}} \frac{1}{|B(x, \varepsilon)|} \int_{B(x, \varepsilon)}|f(y)-f(x)| d \mathscr{L}^{n}(y)=0 \quad \text { for a.e. } x \in \Omega .
$$

To prove (1), fix $x \in \Omega$ for which (3.26) holds. Then, for $\varepsilon>0$ sufficiently small such that $x \in \Omega_{\varepsilon}$, from (3.20), the properties of $\theta_{\varepsilon}$, and (3.26) we have

$$
\begin{aligned}
\left|f_{\varepsilon}(x)-f(x)\right| & \leq \int_{B(x, \varepsilon)}|f(y)-f(x)| \theta_{\varepsilon}(x-y) d \mathscr{L}^{n}(y) \\
& \leq C(n)\|\theta\|_{L^{\infty}(\overline{B(0,1)})} \frac{1}{|B(x, \varepsilon)|} \int_{B(x, \varepsilon)}|f(y)-f(x)| d \mathscr{L}^{n}(y) \stackrel{\varepsilon \rightarrow 0^{+}}{\longrightarrow} 0 .
\end{aligned}
$$

Hence $f_{\varepsilon}(x) \stackrel{\varepsilon \rightarrow 0^{+}}{\longrightarrow} f(x)$. This proves (1).

Next, let $K \subseteq \Omega$ be an arbitrary compact set. Then, for every $\varepsilon \in\left(0, \varepsilon_{K}\right)$, from (3.20) and Fubini's Theorem (recall (3.24)) we have

$$
\begin{aligned}
\int_{K}\left|f_{\varepsilon}(x)\right| d \mathscr{L}^{n}(x) & \leq \int_{K} \int_{B(x, \varepsilon)} \theta_{\varepsilon}(x-y)|f(y)| d \mathscr{L}^{n}(y) d \mathscr{L}^{n}(x) \\
& \leq \int_{K_{\varepsilon}}|f(y)| \int_{B(y, \varepsilon)} \theta_{\varepsilon}(x-y) d \mathscr{L}^{n}(x) d \mathscr{L}^{n}(y) .
\end{aligned}
$$


For the last inequality in (3.28) we have used the fact that

$$
\{(x, y): x \in K,|x-y|<\varepsilon\} \subseteq\left\{(x, y): y \in K_{\varepsilon},|x-y|<\varepsilon\right\}, \quad \forall \varepsilon>0 .
$$

Clearly, (3.28) proves (2).

We are left with proving (3.25). To this end, for each $N \in \mathbb{N}$, define the function

$$
f_{N}:=\left\{\begin{array}{cc}
f & \text { if }|f| \leq N \\
0 & \text { otherwise }
\end{array}\right.
$$

Then from definition and the properties of $f$, for every $N \in \mathbb{N}$, we have $f_{N} \in L_{l o c}^{1}(\Omega)$ and $\left|f_{N}\right| \leq|f|$ a.e. in $\Omega$. Also, since $|f|<\infty$ a.e. in $\Omega$ and the definition of $f_{N}$, it follows that there exists a set $A \subseteq \Omega$ with $\mathscr{L}^{n}(A)=0$ such that for each $x \in \Omega \backslash A$ we have $f_{N}(x)=f(x)$ if $N$ is sufficiently large. Hence, $f_{N} \stackrel{N \rightarrow \infty}{\longrightarrow} f$ for $\mathscr{L}^{n}$-a.e. in $\Omega$. All thees allow us to apply Lebesgue's Dominated Comnergence Theorem and conclude that

$$
f_{N} \stackrel{N \rightarrow \infty}{\longrightarrow} f \quad \text { in } \quad L^{1}(K)
$$

Now pick $\varepsilon_{0} \in\left(0, \varepsilon_{K}\right)$ and, for each $\varepsilon \in\left(0, \varepsilon_{0}\right)$ and each $N \in \mathbb{N}$, write

$$
f_{\varepsilon}-f=\left(f-f_{N}\right)_{\varepsilon}+\left(f_{N}-f\right)+\left(f_{N}\right)_{\varepsilon}-f_{N}
$$

where $\left(f-f_{N}\right)_{\varepsilon}$ and $\left(f_{N}\right)_{\varepsilon}$ should be understood as in (3.20) with $f$ replaced by $f-f_{N}$ and $f_{N}$, respectively. In particular, for each $\varepsilon \in\left(0, \varepsilon_{0}\right)$ and each $N \in \mathbb{N}$, starting from 
(3.32) we may estimate

$$
\begin{aligned}
\left\|f_{\varepsilon}-f\right\|_{L^{1}(K)} & \leq\left\|\left(f-f_{N}\right)_{\varepsilon}\right\|_{L^{1}(K)}+\left\|f_{N}-f\right\|_{L^{1}(K)}+\left\|\left(f_{N}\right)_{\varepsilon}-f_{N}\right\|_{L^{1}(K)} \\
& \leq\left\|f-f_{N}\right\|_{L^{1}\left(K_{\varepsilon}\right)}+\left\|f_{N}-f\right\|_{L^{1}(K)}+\left\|\left(f_{N}\right)_{\varepsilon}-f_{N}\right\|_{L^{1}(K)} \\
& \leq 2\left\|f-f_{N}\right\|_{L^{1}\left(K_{\varepsilon_{0}}\right)}+\left\|\left(f_{N}\right)_{\varepsilon}-f_{N}\right\|_{L^{1}(K)} .
\end{aligned}
$$

For the second inequality in (3.33) we used (2), while the last inequality is immediate since $K_{\varepsilon} \subseteq K_{\varepsilon_{0}}$ for $\varepsilon \in\left(0, \varepsilon_{0}\right)$. Taking the lim sup $\operatorname{su}_{\varepsilon \rightarrow 0^{+}}$of the most extreme sides in (3.33) gives

$$
\limsup _{\varepsilon \rightarrow 0^{+}}\left\|f_{\varepsilon}-f\right\|_{L^{1}(K)} \leq 2\left\|f-f_{N}\right\|_{L^{1}\left(K_{\varepsilon_{0}}\right)}+\limsup _{\varepsilon \rightarrow 0^{+}}\left\|\left(f_{N}\right)_{\varepsilon}-f_{N}\right\|_{L^{1}(K)} .
$$

We claim that for each $N \in \mathbb{N}$ there holds

$$
\left(f_{N}\right)_{\varepsilon} \stackrel{\varepsilon \rightarrow 0^{+}}{\longrightarrow} f_{N} \quad \text { in } \quad L^{1}(K)
$$

Indeed, this is a consequence of (1), the fact that $\left|\left(f_{N}\right)_{\varepsilon}\right| \leq\left\|f_{N}\right\|_{L^{\infty}(\Omega)} \leq N \in L^{1}(K)$ for each $N$, and Lebesgue's Dominated Convergence Theorem. A combination of (3.34) and (3.35) then yields

$$
\limsup _{\varepsilon \rightarrow 0^{+}}\left\|f_{\varepsilon}-f\right\|_{L^{1}(K)} \leq 2\left\|f-f_{N}\right\|_{L^{1}\left(K_{\varepsilon_{0}}\right)} .
$$

At this point, by taking the limit $N \rightarrow \infty$ in (3.36) and invoking (3.31) we arrive at $\limsup \sup _{\varepsilon \rightarrow 0^{+}}\left\|f_{\varepsilon}-f\right\|_{L^{1}(K)}$. This completes the proof of (3.25) and finishes the proof of the lemma.

We close this section by stating and proving an integration by parts formula. 
In the sequel, the $(n-1)$-dimensional Hausdorff measure in $\mathbb{R}^{n}$ will be denoted by $\mathscr{H}^{n-1}$.

Theorem 3.3. Let $\Omega$ be an open subset of $\mathbb{R}^{n}$ with compact Lipschitz boundary and denote its outward unit normal by $v=\left(v_{1}, \ldots, v_{n}\right)$. Let $k \in \mathbb{C}$ be arbitrary. Suppose $u, v \in \mathscr{C}^{0}\left(\bar{\Omega}, C \ell_{m}\right)$ satisfy (when derivatives are computed in the sense of distributions)

$$
(u D),(D v) \in L_{\mathrm{loc}}^{1}\left(\Omega, C l_{m}\right) \quad \text { and } \quad(u D) \odot v+u \odot(D v) \in L^{1}\left(\Omega, C l_{m}\right) .
$$

If $\Omega$ is unbounded, also assume that

$$
\lim _{R \rightarrow \infty} \int_{|x|=R} u(x) \odot \frac{x}{|x|} \odot v(x) d \mathscr{H}^{n-1}(x)=0 .
$$

Then the following integration by parts formula holds:

$$
\begin{gathered}
\int_{\Omega}\left\{\left(u D_{-k}\right)(x) \odot v(x)+u(x) \odot\left(D_{k} v\right)(x)\right\} d \mathscr{L}^{n}(x) \\
=\int_{\partial \Omega} u(x) \odot v(x) \odot v(x) d \mathscr{H}^{n-1}(x) .
\end{gathered}
$$

Proof. With an eye toward applying the Divergence Theorem B.1, define

$$
F_{j}:=u \odot e_{j} \odot v \quad \forall j \in\{1, \ldots, n\},
$$

and

$$
\vec{F}:=\left(F_{j}\right)_{1 \leq j \leq n}
$$

Then, given the current assumptions on $u$ and $v$, we have

$$
\vec{F} \in\left[\mathscr{C}^{0}\left(\bar{\Omega}, C \ell_{m}\right)\right]^{n}
$$


and it is immediate that

$$
\sum_{j=1}^{n} \frac{x_{j}}{|x|} F_{j}(x)=u(x) \odot \frac{x}{|x|} \odot v(x) \quad \text { for every } x \in \Omega
$$

In particular, from (3.43) and the assumption (3.38) when $\Omega$ is unbounded, it follows that $\vec{F}$ satisfies (B.2) in the case when $\Omega$ is unbounded. In order to apply Theorem B.1, it remains to show that $\operatorname{div} \vec{F}$, taken in the sense of distributions in $\mathcal{D}^{\prime}\left(\Omega, C \ell_{m}\right)$, satisfies $\operatorname{div} \vec{F} \in L^{1}\left(\Omega, C \ell_{m}\right)$.

Pick an arbitrary test function $\varphi \in \mathscr{C}_{0}^{\infty}(\Omega)$. Then

$$
\begin{aligned}
\langle\operatorname{div} \vec{F}, \varphi\rangle\rangle & =-\sum_{j=1}^{n}\left\langle\left\langle F_{j}, \partial_{j} \varphi\right\rangle\right\rangle \\
& =-\sum_{j=1}^{n} \int_{\Omega} F_{j}(x) \partial_{j} \varphi(x) d \mathscr{L}^{n}(x) \\
& =-\sum_{j=1}^{n} \int_{\Omega} u(x) \odot e_{j} \odot\left(\partial_{j} \varphi\right)(x) v(x) d \mathscr{L}^{n}(x) .
\end{aligned}
$$

Since $\operatorname{supp}(\varphi) \subseteq \Omega$ is compact, $\operatorname{dist}(\operatorname{supp}(\varphi), \partial \Omega)>0$. Fix $\varepsilon \in(0, \operatorname{dist}(\operatorname{supp}(\varphi), \partial \Omega))$ arbitrary and define

$$
\Omega_{\varepsilon}:=\{x \in \Omega: \operatorname{dist}(x, \partial \Omega)>\varepsilon\} .
$$

Recalling the function $\theta_{\varepsilon}$ from (3.18), consider

$$
\begin{aligned}
v_{\varepsilon}(x): & =\left(v * \theta_{\varepsilon}\right)(x)=\int_{B(0, \varepsilon)} v(x-y) \theta_{\varepsilon}(y) d \mathscr{L}^{n}(y) \\
& =\int_{B(x, \varepsilon)} v(y) \theta_{\varepsilon}(x-y) d \mathscr{L}^{n}(y), \quad \forall x \in \Omega_{\varepsilon} .
\end{aligned}
$$


Then

$$
v_{\varepsilon} \in \mathscr{C}^{\infty}\left(\Omega_{\varepsilon}, C \ell_{m}\right) \quad \text { and } \quad \varphi v_{\varepsilon} \in \mathscr{C}_{0}^{\infty}\left(\Omega, C \ell_{m}\right)
$$

Also, by Lemma 3.2, we have

$$
v_{\varepsilon} \stackrel{\varepsilon \rightarrow 0^{+}}{\longrightarrow} v \text { uniformly on compact subsets of } \Omega \text {. }
$$

Thus, based on (3.48), the fact that $\varphi$ is compactly supported, and that $u \in$ $L^{\infty}\left(\Omega, C \ell_{m}\right)$, we may apply Lebesgue's Dominated Convergence Theorem followed by the product rule to write

$$
\begin{aligned}
\langle\langle\operatorname{div} \vec{F}, \varphi\rangle= & -\sum_{j=1}^{n} \int_{\Omega} u(x) \odot e_{j} \odot\left(\partial_{j} \varphi\right)(x) v(x) d \mathscr{L}^{n}(x) \\
= & -\lim _{\varepsilon \rightarrow 0^{+}} \sum_{j=1}^{n} \int_{\Omega} u(x) \odot e_{j} \odot\left(\partial_{j} \varphi\right)(x) v_{\varepsilon}(x) d \mathscr{L}^{n}(x) \\
= & -\lim _{\varepsilon \rightarrow 0^{+}} \sum_{j=1}^{n} \int_{\Omega} u(x) \odot e_{j} \odot \partial_{j}\left(\varphi v_{\varepsilon}\right)(x) d \mathscr{L}^{n}(x) \\
& +\lim _{\varepsilon \rightarrow 0^{+}} \sum_{j=1}^{n} \int_{\Omega} u(x) \odot e_{j} \odot \varphi(x)\left(\partial_{j} v_{\varepsilon}\right)(x) d \mathscr{L}^{n}(x) \\
= & : I+I I .
\end{aligned}
$$

At this point we find it useful to extend the definition of (3.2) to include $C \ell_{m^{-}}$ valued test functions as follows:

$$
\langle\langle w, \psi\rangle\rangle:=\sum_{I} \sum_{J} \mathcal{D}^{\prime}(\Omega)\left\langle w_{I}, \psi_{J}\right\rangle_{\mathcal{D}(\Omega)} e_{I} \odot e_{J}
$$


for every $w=\sum_{I} w_{I} e_{I} \in \mathcal{D}^{\prime}\left(\Omega, C \ell_{m}\right)$ and $\psi=\sum_{I} \psi_{I} e_{I} \in \mathscr{C}_{0}^{\infty}\left(\Omega, C \ell_{m}\right)$.

Under this convention, using (3.37), (3.47), and (3.48), we may write

$$
\begin{aligned}
I & =-\lim _{\varepsilon \rightarrow 0^{+}} \sum_{j=1}^{n} \int_{\Omega} u(x) \odot e_{j} \odot \partial_{j}\left(\varphi v_{\varepsilon}\right)(x) d \mathscr{L}^{n}(x) \\
& =-\lim _{\varepsilon \rightarrow 0^{+}} \sum_{j=1}^{n}\left\langle\left\langle u \odot e_{j}, \partial_{j}\left(\varphi v_{\varepsilon}\right)\right\rangle\right\rangle \\
& =\lim _{\varepsilon \rightarrow 0^{+}} \sum_{j=1}^{n}\left\langle\left\langle\partial_{j} u \odot e_{j}, \varphi v_{\varepsilon}\right\rangle\right\rangle=\lim _{\varepsilon \rightarrow 0^{+}}\left\langle\left\langle u D, \varphi v_{\varepsilon}\right\rangle\right\rangle \\
& =\lim _{\varepsilon \rightarrow 0^{+}} \int_{\Omega}(u D)(x) \odot v_{\varepsilon}(x) \varphi(x) d \mathscr{L}^{n}(x) .
\end{aligned}
$$

Furthermore, one more use of the fact that $(u D) \in L_{\text {loc }}^{1}\left(\Omega, C \ell_{m}\right)$ and $v_{\varepsilon} \varphi \in \mathscr{C}_{0}^{\infty}\left(\Omega, C \ell_{m}\right)$, combined with Lebesgue's Dominated Convergence Theorem, yields

$$
I=\int_{\Omega}(u D)(x) \odot v(x) \varphi(x) d \mathscr{L}^{n}(x)=\langle\langle(u D) \odot v, \varphi\rangle\rangle .
$$

Next we analyze

$$
\begin{aligned}
I I & =\lim _{\varepsilon \rightarrow 0^{+}} \sum_{j=1}^{n} \int_{\Omega} u(x) \odot e_{j} \odot \varphi(x)\left(\partial_{j} v_{\varepsilon}\right)(x) d \mathscr{L}^{n}(x) \\
& =\lim _{\varepsilon \rightarrow 0^{+}} \int_{\Omega} u(x) \odot\left(D v_{\varepsilon}\right)(x) \varphi(x) d \mathscr{L}^{n}(x) .
\end{aligned}
$$

We claim that

$$
D v_{\varepsilon}=(D v)_{\varepsilon} \text { in } \Omega_{\varepsilon} .
$$


To prove this claim, fix $x \in \Omega_{\varepsilon}$ arbitrary. Recalling (3.46), we may compute

$$
\begin{aligned}
\partial_{j} v_{\varepsilon}(x) & =\int_{B(x, \varepsilon)} v(y) \partial_{x_{j}}\left[\theta_{\varepsilon}(x-y)\right] d \mathscr{L}^{n}(y) \\
& =-\int_{B(x, \varepsilon)} v(y) \partial_{y_{j}}\left[\theta_{\varepsilon}(x-y)\right] d \mathscr{L}^{n}(y) \\
& =-\left\langle\left\langle v, \partial_{j}\left[\theta_{\varepsilon}(x-\cdot)\right]\right\rangle\right\rangle \\
& =\left\langle\left\langle\partial_{j} v, \theta_{\varepsilon}(x-\cdot)\right\rangle\right\rangle .
\end{aligned}
$$

Hence,

$$
\begin{aligned}
D\left[v_{\varepsilon}(x)\right] & =\left\langle\left\langle D v, \theta_{\varepsilon}(x-\cdot)\right\rangle\right\rangle \\
& =\int_{B(x, \varepsilon)}(D v)(y) \theta_{\varepsilon}(x-y) d \mathscr{L}^{n}(y) \\
& =(D v)_{\varepsilon}(x)
\end{aligned}
$$

completing the proof of claim (3.54). In addition, by Lemma 3.2,

$$
(D v)_{\varepsilon} \stackrel{\varepsilon \rightarrow 0^{+}}{\longrightarrow} D v \quad \text { in } \quad L_{\mathrm{loc}}^{1}\left(\Omega, C \ell_{m}\right)
$$

Making use of (3.54) and (3.57) in (3.53), and then once again using Lebesgue's Dominated Convergence Theorem, results in

$$
\begin{aligned}
I I & =\lim _{\varepsilon \rightarrow 0^{+}} \int_{\Omega} u(x) \odot(D v)_{\varepsilon}(x) \varphi(x) d \mathscr{L}^{n}(x) \\
& =\int_{\Omega} u(x) \odot(D v)(x) \varphi(x) d \mathscr{L}^{n}(x) \\
& =\langle\langle u \odot(D v), \varphi\rangle\rangle .
\end{aligned}
$$


Returning now to (3.49) together with (3.52) and (3.58) we see that

$$
\langle\langle\operatorname{div} \vec{F}, \varphi\rangle\rangle=\langle\langle(u D) \odot v+u \odot(D v), \varphi\rangle\rangle,
$$

from which we conclude that

$$
\operatorname{div} \vec{F}=(u D) \odot v+u \odot(D v) \quad \text { in } \quad \mathcal{D}^{\prime}\left(\Omega, C \ell_{m}\right)
$$

In concert with (3.37), formula (3.60) implies $\operatorname{div} \vec{F} \in L^{1}\left(\Omega, C \ell_{m}\right)$. Since $\vec{F}$ satisfies all hypotheses of the Divergence Theorem B.1, the latter applies and gives

$$
\begin{gathered}
\int_{\Omega}\{(u D)(x) \odot v(x)+u(x) \odot(D v)(x)\} d \mathscr{L}^{n}(x) \\
=\int_{\partial \Omega} u(x) \odot v(x) \odot v(x) d \mathscr{H}^{n-1}(x) .
\end{gathered}
$$

Recalling (2.25) and (2.26), we see that

$$
\begin{aligned}
(u D)(x) \odot v(x)+u(x) \odot(D v)(x) & \\
& =(u D)(x) \odot v(x)-u(x) \odot k e_{j} \odot v(x)+u(x) \odot(D v)(x)+u(x) \odot k e_{j} \odot v(x) \\
& =\left[(u D)(x)-u(x) \odot k e_{j}\right] \odot v(x)+u(x) \odot\left[(D v)(x)+k e_{j} \odot v(x)\right] \\
& =\left(u D_{-k}\right)(x) \odot v(x)+u(x) \odot\left(D_{k} v\right)(x), \quad \forall x \in \Omega .
\end{aligned}
$$

Combining (3.61) with (3.62) yields (3.39), completing the proof of the theorem. 


\section{The radiating fundamental solution for the Helmholtz operator}

Let $n \in \mathbb{N}$ satisfy $n \geq 2$ and suppose $k \in(0, \infty)$. Denote by $H_{\lambda}^{(1)}(\cdot)$ the Hankel function of the first kind with index $\lambda \in \mathbb{R}$ (cf. $[1, \S 9.1]$ ) and consider the function

$$
\Phi_{k}(x):=c_{n} k^{(n-2) / 2} \frac{H_{(n-2) / 2}^{(1)}(k|x|)}{|x|^{(n-2) / 2}}, \quad \forall x \in \mathbb{R}^{n} \backslash\{0\},
$$

where $c_{n}$ is the constant defined by

$$
c_{n}:=\frac{1}{4 i(2 \pi)^{(n-2) / 2}} .
$$

The function $\Phi_{k}$ from (4.1) plays an important role in the context of considering the Helmholtz operator $\Delta+k^{2}$ in $\mathbb{R}^{n}$. The main properties of $\Phi_{k}$ which are relevant for us in the sequel are collected in the next theorem. For proof and other related results see [9].

Theorem 4.1. Suppose $n \in \mathbb{N}, n \geq 2$, fix $k \in(0, \infty)$, and recall the function $\Phi_{k}$ defined in (4.1). Then the following are true.

(1) $\Phi_{k} \in \mathscr{C}^{\infty}\left(\mathbb{R}^{n} \backslash\{0\}\right)$ and $\left(\Delta+k^{2}\right) \Phi_{k}=0$ point-wise in $\mathbb{R}^{n} \backslash\{0\}$.

(2) $\Phi_{k}$ is a locally integrable function in $\mathbb{R}^{n}$ and is the unique distribution in $\mathbb{R}^{n}$ which is a fundamental solution of the Helmholtz operator $\Delta+k^{2}$ in $\mathbb{R}^{n}$ and also satisfies Sommerfeld's radiation condition.

(3) Set

$$
b_{n, k}:=c_{n} k^{(n-3) / 2}\left(\frac{2}{\pi}\right)^{1 / 2} e^{-i \pi(n-1) / 4}=\frac{k^{(n-3) / 2} e^{-i \pi(n+1) / 4}}{2^{(n+1) / 2} \pi^{(n-1) / 2}} .
$$


Then for each multi-index $\alpha \in \mathbb{N}_{0}^{n}$ one has

$$
\begin{aligned}
\left(\partial^{\alpha} \Phi_{k}\right)(x-y) & =b_{n, k} \frac{e^{i k|x|} e^{-i k\langle y, \widehat{x}\rangle}}{|x|^{(n-1) / 2}}(i k \widehat{x})^{\alpha}+O\left(|x|^{-(n+1) / 2}\right) \\
& =(i k \widehat{x})^{\alpha} \Phi_{k}(x-y)+O\left(|x|^{-(n+1) / 2}\right) \quad \text { as }|x| \rightarrow \infty,
\end{aligned}
$$

uniformly for $y$ in compact subsets of $\mathbb{R}^{n}$, and

$$
\begin{aligned}
\left(\partial^{\alpha} \Phi_{k}\right)(x-y) & =b_{n, k} \frac{e^{i k|y|} e^{-i k\langle x, \widehat{y}\rangle}}{|y|^{(n-1) / 2}}(-i k \widehat{y})^{\alpha}+O\left(|y|^{-(n+1) / 2}\right) \\
& =(-i k \widehat{y})^{\alpha} \Phi_{k}(x-y)+O\left(|y|^{-(n+1) / 2}\right) \quad \text { as }|y| \rightarrow \infty,
\end{aligned}
$$

uniformly for $x$ in compact subsets of $\mathbb{R}^{n}$.

In particular, for each multi-index $\alpha \in \mathbb{N}_{0}^{n}$ one has

$$
\begin{gathered}
\left(\partial^{\alpha} \Phi_{k}\right)(x-y)=O\left(|x|^{-(n-1) / 2}\right) \text { as }|x| \rightarrow \infty, \\
\text { uniformly for } y \text { in compact subsets of } \mathbb{R}^{n},
\end{gathered}
$$

and

$$
\begin{gathered}
\left(\partial^{\alpha} \Phi_{k}\right)(x-y)=O\left(|y|^{-(n-1) / 2}\right) \quad \text { as }|y| \rightarrow \infty, \\
\text { uniformly for } x \text { in compact subsets of } \mathbb{R}^{n} .
\end{gathered}
$$

(4) For any two multi-indices $\alpha, \beta \in \mathbb{N}_{0}^{n}$ one has

$$
\begin{gathered}
\left(\partial^{\alpha+\beta} \Phi_{k}\right)(x-y)=(i k \widehat{x})^{\alpha}\left(\partial^{\beta} \Phi_{k}\right)(x-y)+O\left(|x|^{-(n+1) / 2}\right) \text { as }|x| \rightarrow \infty, \\
\text { uniformly for } y \text { in compact subsets of } \mathbb{R}^{n},
\end{gathered}
$$


and

$$
\begin{gathered}
\left(\partial^{\alpha+\beta} \Phi_{k}\right)(x-y)=(-i k \widehat{y})^{\alpha}\left(\partial^{\beta} \Phi_{k}\right)(x-y)+O\left(|y|^{-(n+1) / 2}\right) \text { as }|y| \rightarrow \infty, \\
\text { uniformly for } x \text { in compact subsets of } \mathbb{R}^{n} .
\end{gathered}
$$

In particular, for any $\alpha \in \mathbb{N}_{0}^{n}$ and any $j, \ell \in\{1, \ldots, n\}$,

$$
\begin{gathered}
\widehat{x}_{j}\left(\partial_{\ell} \partial^{\alpha} \Phi_{k}\right)(x-y)-\widehat{x}_{\ell}\left(\partial_{j} \partial^{\alpha} \Phi_{k}\right)(x-y)=O\left(|x|^{-(n+1) / 2}\right) \text { as }|x| \rightarrow \infty, \\
\text { uniformly for } y \text { in compact subsets of } \mathbb{R}^{n},
\end{gathered}
$$

and

$$
\begin{gathered}
\widehat{y}_{j}\left(\partial_{\ell} \partial^{\alpha} \Phi_{k}\right)(x-y)-\widehat{y}_{\ell}\left(\partial_{j} \partial^{\alpha} \Phi_{k}\right)(x-y)=O\left(|y|^{-(n+1) / 2}\right) \text { as }|y| \rightarrow \infty, \\
\text { uniformly for } x \text { in compact subsets of } \mathbb{R}^{n} .
\end{gathered}
$$

(5) There holds

$$
\begin{aligned}
\left(D_{k} \Phi_{k}\right)(x-y) & =\Phi_{k}(x-y)\left(i k \widehat{x}+k e_{n+1}\right)+O\left(|x|^{-(n+1) / 2}\right) \\
& =b_{n, k} \frac{e^{i k|x|} e^{-i k\langle y, \widehat{x}\rangle}}{|x|^{(n-1) / 2}} k\left(i \widehat{x}+e_{n+1}\right)+O\left(|x|^{-(n+1) / 2}\right) \quad \text { as } \quad|x| \rightarrow \infty,
\end{aligned}
$$

uniformly for $y$ in compact subsets of $\mathbb{R}^{n}$, and

$$
\begin{aligned}
\left(D_{k} \Phi_{k}\right)(x-y) & =\Phi_{k}(x-y)\left(-i k \widehat{y}+k e_{n+1}\right)+O\left(|y|^{-(n+1) / 2}\right) \\
& =b_{n, k} \frac{e^{i k|y|} e^{-i k\langle x, \widehat{y}\rangle}}{|y|^{(n-1) / 2}} k\left(-i \widehat{y}+e_{n+1}\right)+O\left(|y|^{-(n+1) / 2}\right) \quad \text { as } \quad|y| \rightarrow \infty
\end{aligned}
$$

uniformly for $x$ in compact subsets of $\mathbb{R}^{n}$. 
(6) For every multi-index $\alpha \in \mathbb{N}_{0^{\prime}}^{n}$

$$
\begin{gathered}
\left\langle\widehat{x},\left[\nabla\left(\partial^{\alpha} \Phi_{k}\right)\right](x-y)\right\rangle-i k\left(\partial^{\alpha} \Phi_{k}\right)(x-y)=O\left(|x|^{-(n+1) / 2}\right) \text { as }|x| \rightarrow \infty, \\
\text { uniformly for y in compact subsets of } \mathbb{R}^{n} .
\end{gathered}
$$

As a consequence, for each $y \in \mathbb{R}^{n}$ fixed,

$$
\left(\partial^{\alpha} \Phi_{k}\right)(\cdot-y) \text { satisfies Sommerfeld's radiation condition (1.1). }
$$

(7) Pick some $R \in(0, \infty)$. Then there exists some constant $C=C(R, n, k) \in(0, \infty)$ such that the function $\Phi_{k}$ satisfies the following estimates for every $x \in B(0, R) \backslash\{0\}$ :

$$
\left|\Phi_{k}(x)\right| \leq\left\{\begin{array}{ll}
C|\ln | x||, & n=2, \\
C|x|^{2-n}, & n \geq 3,
\end{array} \quad \text { and } \quad\left|\left(\nabla \Phi_{k}\right)(x)\right| \leq C|x|^{1-n}\right.
$$

(8) For each $x \in \mathbb{R}^{n}$ fixed, there holds

$$
\left(\partial_{j} \Phi_{k}\right)(x-\cdot)=-\partial_{j}\left[\Phi_{k}(x-\cdot)\right] \quad \text { in } \mathcal{D}^{\prime}\left(\mathbb{R}^{n}\right), \quad \forall j \in\{1, \ldots, n\}
$$

In order to state and prove a result regarding additional properties of the function $\Phi_{k}$ we need to introduce an auxiliary function which we call the profile function of $\Phi_{k}$. Specifically, for each $k \in(0, \infty)$ and each $n \in \mathbb{N}$, consider the function

$$
\Psi_{k}^{(n)}:(0, \infty) \rightarrow \mathbb{C}, \quad \Psi_{k}^{(n)}(\rho):=c_{n} k^{(n-2) / 2} \frac{H_{(n-2) / 2}^{(1)}(k \rho)}{\rho^{(n-2) / 2}}, \quad \forall \rho \in(0, \infty),
$$

where $c_{n}$ is as in (4.1). 
Lemma 4.2. Let $k \in(0, \infty)$ and $n \in \mathbb{N}$ and consider $\Psi_{k}^{(n)}$ as in (4.18). Then the following are true.

(1) $\Phi_{k}(x)=\Psi_{k}^{(n)}(|x|)$ for every $x \in \mathbb{R}^{n} \backslash\{0\}$.

(2) $\frac{d}{d \rho}\left[\Psi_{k}^{(n)}(\rho)\right]=-2 \pi \rho \Psi_{k}^{(n+2)}(\rho)$ for all $\rho \in(0, \infty)$.

(3) For each $j \in\{1, \ldots, n\}$, one has $\left(\partial_{j} \Phi_{k}\right)(x)=-2 \pi x_{j} \Psi_{k}^{(n+2)}(|x|)$ at every $x \in \mathbb{R}^{n} \backslash\{0\}$. Consequently,

$$
x_{r}\left(\partial_{s} \Phi_{k}\right)(x)-x_{s}\left(\partial_{r} \Phi_{k}\right)(x)=0 \text { for all } r, s \in\{1, \ldots, n\} \text { and } x \in \mathbb{R}^{n} \backslash\{0\} \text {. }
$$

In particular, given any $r, s \in\{1, \ldots, n\}$, for every $x, y \in \mathbb{R}^{n}$ with $x \neq y$ one has

$$
x_{r}\left(\partial_{s} \Phi_{k}\right)(x-y)-x_{s}\left(\partial_{r} \Phi_{k}\right)(x-y)=y_{r}\left(\partial_{s} \Phi_{k}\right)(x-y)-y_{s}\left(\partial_{r} \Phi_{k}\right)(x-y)
$$

(4) $\frac{d}{d k}\left[\Psi_{k}^{(n)}(\rho)\right]=\frac{k}{2 \pi} \Psi_{k}^{(n-2)}(\rho)$ for $\rho \in(0, \infty)$.

Proof. The identity in (1) is immediate from definitions. Also, using (4.18), (A.5), and the fact that $c_{n}=2 \pi c_{n+2}$, we have

$$
\begin{aligned}
\frac{d}{d \rho} & {\left[\Psi_{k}^{(n)}(\rho)\right]=c_{n} k^{(n-2) / 2} \frac{2-n}{2} \frac{H_{(n-2) / 2}^{(1)}(k \rho)}{\rho^{(n-2) / 2+1}}+\frac{c_{n} k^{(n-2) / 2+1}}{\rho^{(n-2) / 2}}\left(\frac{d}{d r} H_{(n-2) / 2}^{(1)}\right)(k \rho) } \\
& =\frac{c_{n} k^{(n-2) / 2}}{\rho^{n / 2}}\left(\frac{2-n}{2} H_{(n-2) / 2}^{(1)}(k \rho)-k \rho H_{(n-2) / 2+1}^{(1)}(k \rho)+k \rho \frac{(n-2) / 2}{k \rho} H_{(n-2) / 2}^{(1)}(k \rho)\right) \\
& =-\frac{c_{n} k^{n / 2} \rho}{\rho^{n / 2}} H_{n / 2}^{(1)}(k \rho)=-2 \pi \rho \Psi_{k}^{(n+2)}(\rho), \quad \forall \rho \in(0, \infty) .
\end{aligned}
$$

This proves the identity in (2). Combining this with the chain rule we have

$$
\left(\partial_{j} \Phi_{k}\right)(x)=\left.\frac{d}{d \rho}\left[\Psi_{k}^{(n)}(\rho)\right]\right|_{\rho=|x|} \frac{x_{j}}{|x|}=-2 \pi x_{j} \Psi_{k}^{(n+2)}(|x|),
$$


which proves (3). Finally, based on (4.18) and (A.4) we have

$$
\begin{aligned}
\frac{d}{d k}\left[\Psi_{k}^{(n)}(\rho)\right]= & c_{n} k^{(n-2) / 2-1} \frac{n-2}{2} \frac{H_{(n-2) / 2}^{(1)}(k \rho)}{\rho^{(n-2) / 2}}+\frac{c_{n} k^{(n-2) / 2} \rho}{\rho^{(n-2) / 2}}\left(\frac{d}{d r} H_{(n-2) / 2}^{(1)}\right)(k \rho) \\
= & \frac{c_{n} k^{(n-4) / 2}}{\rho^{(n-2) / 2}}\left(\frac{n-2}{2} H_{(n-2) / 2}^{(1)}(k \rho)+k \rho H_{(n-2) / 2-1}^{(1)}(k \rho)\right. \\
& \left.\quad k \rho \frac{(n-2) / 2}{k \rho} H_{(n-2) / 2}^{(1)}(k \rho)\right) \\
= & \frac{c_{n} k^{(n-4) / 2} k \rho}{\rho^{(n-2) / 2}} H_{(n-4) / 2}^{(1)}(k \rho)=\frac{k}{2 \pi} \Psi_{k}^{(n-2)}(\rho), \quad \forall \rho \in(0, \infty),
\end{aligned}
$$

where for the last equality we also used that $c_{n}=\frac{c_{n-2}}{2 \pi}$. This completes the proof of the lemma. 


\section{Fundamental solutions for iterated Helmholtz oper- ators}

Throughout this section fix $n \in \mathbb{N}$ satisfying $n \geq 2$ and a wave number $k \in(0, \infty)$. Recall the fundamental solution for the Helmholtz operator $\Delta+k^{2}$ in $\mathbb{R}^{n}$ from (4.1) and the constant $c_{n}$ from (4.2). Seeking a fundamental solution of the iterated Helmholtz operator $\left(\Delta+k^{2}\right)^{N}$, where $N \in \mathbb{N}$, we $\operatorname{set}^{1}$

$$
\Phi_{k}^{(N)}(x):=\frac{(-1)^{N-1} c_{n} k^{(n-2 N) / 2} H_{(n-2 N) / 2}^{(1)}(k|x|)}{2^{N-1}(N-1) !|x|^{(n-2 N) / 2}}, \quad \forall x \in \mathbb{R}^{n} \backslash\{0\} .
$$

Since $\Phi_{k}^{(N)}(x)$ is a radial function, we find it convenient to also define

$$
\phi_{k}^{(N)}(r):=\frac{(-1)^{N-1} c_{n} k^{(n-2 N) / 2} H_{(n-2 N) / 2}^{(1)}(k r)}{2^{N-1}(N-1) ! r^{(n-2 N) / 2}}, \quad \forall r \in(0, \infty) .
$$

In particular, $\Phi_{k}^{(N)}(x)=\phi_{k}^{(N)}(|x|)$ for all $x \in \mathbb{R}^{n} \backslash\{0\}$. Also, since for each $\lambda \in \mathbb{R}$ the Hankel function $H_{\lambda}^{(1)}(\cdot)$ is an analytic function on $\mathbb{C} \backslash(-\infty, 0]$, i.e. the complex plane with a branch cut along the non-positive real axis (cf. [18]), we have that $\Phi_{k}^{(N)} \in \mathscr{C}^{\infty}\left(\mathbb{R}^{n} \backslash 0\right)$ for every $N \in \mathbb{N}$. Some differentiation formulas for $\Phi_{k}^{(N)}$ which will be useful in the sequal are collected in the following three lemmas.

Lemma 5.1. Let $k \in(0, \infty)$ and $N \in \mathbb{N}$. Then the following recursive property holds:

$$
D \Phi_{k}^{(N+1)}(x)=\nabla \Phi_{k}^{(N+1)}(x)=\frac{\Phi_{k}^{(N)}(x)}{2 N} x, \quad \forall x \in \mathbb{R}^{n} \backslash\{0\} .
$$

\footnotetext{
${ }^{1}$ Notice: when $N=1$ we have $\Phi_{k}^{(1)}(x) \equiv \Phi_{k}(x)$ on $\mathbb{R}^{n} \backslash\{0\}$.
} 
Moreover,

$$
D \Phi_{k}^{(N)}(x)=\frac{(-1)^{N} c_{n} k^{(n-2 N+2) / 2} H_{(n-2 N+2) / 2}^{(1)}(k|x|)}{2^{N-1}(N-1) !|x|^{(n-2 N+2) / 2}} x, \quad \forall x \in \mathbb{R}^{n} \backslash\{0\} .
$$

In addition,

$$
\begin{aligned}
D_{k} \Phi_{k}^{(N)}(x) & =\frac{(-1)^{N} c_{n} k^{(n-2 N+2) / 2}}{2^{N-1}(N-1) !|x|^{(n-2 N) / 2}}\left[H_{(n-2 N+2) / 2}^{(1)}(k|x|) \widehat{x}-H_{(n-2 N) / 2}^{(1)}(k|x|) e_{n+1}\right], \\
D_{-k} \Phi_{k}^{(N)}(x) & =\frac{(-1)^{N} c_{n} k^{(n-2 N+2) / 2}}{2^{N-1}(N-1) !|x|^{(n-2 N) / 2}}\left[H_{(n-2 N+2) / 2}^{(1)}(k|x|) \widehat{x}+H_{(n-2 N) / 2}^{(1)}(k|x|) e_{n+1}\right],
\end{aligned}
$$

pointwise at every $x \in \mathbb{R}^{n} \backslash\{0\}$.

Proof. First, we make the observation that, for each $p \in \mathbb{R}$, we have

$$
\nabla\left[|x|^{p}\right]=\sum_{j=1}^{n} \frac{\partial}{\partial x_{j}}\left(x_{1}^{2}+\ldots+x_{n}^{2}\right)^{p / 2} e_{j}=p|x|^{(p-2)} x=p|x|^{(p-1)} \widehat{x}, \quad \forall x \in \mathbb{R}^{n} \backslash\{0\}
$$

Second, to streamline our computations, for each $\lambda \in \mathbb{R}$, we define the function

$$
F_{\lambda}(r):=\frac{H_{\lambda}^{(1)}(r)}{r^{\lambda}}, \quad \forall r \in(0, \infty)
$$

Then (A.3) implies

$$
\frac{d}{d r}\left[F_{\lambda}(r)\right]=-r F_{\lambda+1}(r), \quad \forall r \in(0, \infty)
$$

Hence, (5.9), the chain rule, and (5.7) allow us to compute

$$
\begin{aligned}
\nabla F_{\lambda}(k|x|) & =-k|x| F_{\lambda+1}(k|x|) \nabla[k|x|]=-k|x| F_{\lambda+1}(k|x|) k \widehat{x} \\
& =-k^{2} F_{\lambda+1}(k|x|) x, \quad \forall x \in \mathbb{R}^{n} \backslash\{0\} .
\end{aligned}
$$


Starting with (5.1) and then making use of the notation introduced in (5.8) combined with formula (5.10), we may compute

$$
\begin{aligned}
D \Phi_{k}^{(N+1)}(x)=\nabla \Phi_{k}^{(N+1)}(x) & =\nabla\left[\frac{(-1)^{N} c_{n} k^{(n-2 N-2) / 2} H_{(n-2 N-2) / 2}^{(1)}(k|x|)}{2^{N} N !|x|^{(n-2 N-2) / 2}}\right] \\
& =\frac{(-1)^{N} c_{n} k^{(n-2 N-2)}}{2^{N} N !} \nabla F_{(n-2 N-2) / 2}(k|x|) \\
& =\frac{(-1)^{N+1} c_{n} k^{(n-2 N)}}{2^{N} N !} F_{(n-2 N) / 2}(k|x|) x \\
& =\frac{(-1)^{(N+1)} c_{n} k^{(n-2 N) / 2} H_{(n-2 N) / 2}^{(1)}(k|x|)}{2^{N} N !|x|^{(n-2 N) / 2}} x
\end{aligned}
$$

for every $x \in \mathbb{R}^{n} \backslash\{0\}$. In light of (5.1), it is immediate that the last expression in (5.11) becomes

$$
D \Phi_{k}^{(N+1)}(x)=\frac{\Phi_{k}^{(N)}(x)}{2 N} x, \quad \forall x \in \mathbb{R}^{n} \backslash\{0\} .
$$

This proves (5.3). The formula for $D \Phi_{k}^{(N+1)}(x)$ obtained in (5.11) also yields (5.4) in the case $N \geq 2$ (seen by replacing $N+1$ by $N$ in (5.11)). To prove (5.4) for $N=1$, we employing again (5.8) and formula (5.10), to write

$$
\begin{aligned}
D \Phi_{k}^{(1)}(x)=\nabla \Phi_{k}^{(1)}(x) & =\nabla \frac{c_{n} k^{(n-2) / 2} H_{(n-2) / 2}^{(1)}(k|x|)}{|x|^{(n-2) / 2}} \\
& =c_{n} k^{n-2} \nabla F_{(n-2) / 2}(k|x|) \\
& =-c_{n} k^{n} F_{n / 2}(k|x|) x \\
& =-c_{n} k^{n / 2} \frac{H_{n / 2}^{(1)}(k|x|)}{|x|^{n / 2}} x, \quad \forall x \in \mathbb{R}^{n} \backslash\{0\} .
\end{aligned}
$$

This completes the proof of (5.4). 
Next, (2.24), (5.4), and (5.1) allow us to write

$$
\begin{aligned}
D_{k} \Phi_{k}^{(N)}(x)= & D \Phi_{k}^{(N)}(x)+k \Phi_{k}^{(N)}(x) e_{n+1} \\
= & \frac{(-1)^{N} c_{n} k^{(n-2 N+2) / 2} H_{(n-2 N+2) / 2}^{(1)}(k|x|)}{2^{N-1}(N-1) !|x|^{(n-2 N+2) / 2}} x \\
& \quad+\frac{(-1)^{N-1} c_{n} k^{(n-2 N) / 2} H_{(n-2 N) / 2}^{(1)}(k|x|)}{2^{N-1}(N-1) !|x|^{(n-2 N) / 2}} e_{n+1} \\
= & \frac{(-1)^{N} c_{n} k^{(n-2 N+2) / 2}}{2^{N-1}(N-1) !|x|^{(n-2 N) / 2}}\left[H_{(n-2 N+2) / 2}^{(1)}(k|x|) \widehat{x}-H_{(n-2 N) / 2}^{(1)}(k|x|) e_{n+1}\right],
\end{aligned}
$$

$\forall x \in \mathbb{R}^{n} \backslash\{0\}$, which yields (5.5). Similarly,

$$
\begin{aligned}
D_{-k} \Phi_{k}^{(N)}(x) & =D \Phi_{k}^{(N)}(x)-k \Phi_{k}^{(N)}(x) e_{n+1} \\
& =\frac{(-1)^{N} C_{n} k^{(n-2 N+2) / 2}}{2^{N-1}(N-1) !|x|^{(n-2 N) / 2}}\left[H_{(n-2 N+2) / 2}^{(1)}(k|x|) \widehat{x}+H_{(n-2 N) / 2}^{(1)}(k|x|) e_{n+1}\right],
\end{aligned}
$$

$\forall x \in \mathbb{R}^{n} \backslash\{0\}$. This gives (5.6) and finishes the proof of the lemma.

Lemma 5.2. For any $N \in \mathbb{N}, k \in(0, \infty)$, the following recursive property holds:

$$
\Phi_{k}^{(N+1)}(x)=\frac{-1}{2 N k} \frac{d}{d k} \Phi_{k}^{(N)}(x), \quad \forall x \in \mathbb{R}^{n} \backslash\{0\} .
$$


Proof. A direct computation yields

$$
\begin{aligned}
\frac{-1}{2 N k} \frac{d}{d k} \Phi_{k}^{(N)}(x)= & \frac{(-1)^{N} c_{n}}{2^{N} N ! k|x|^{(n-2 N) / 2}} \frac{d}{d k}\left[k^{(n-2 N) / 2} H_{(n-2 N) / 2}^{(1)}(k|x|)\right] \\
= & \frac{(-1)^{N} c_{n}}{2^{N} N ! k|x|^{(n-2 N) / 2}}\left[\frac{n-2 N}{2} k^{(n-2 N-2) / 2} H_{(n-2 N) / 2}^{(1)}(k|x|)\right. \\
& \left.+k^{(n-2 N) / 2} \frac{d}{d k} H_{(n-2 N) / 2}^{(1)}(k|x|)\right] \\
= & \frac{(-1)^{N} c_{n}}{2^{N} N ! k|x|^{(n-2 N) / 2}}\left[\frac{n-2 N}{2} k^{(n-2 N-2) / 2} H_{(n-2 N) / 2}^{(1)}(k|x|)\right. \\
& \left.+k^{(n-2 N) / 2}|x|\left(H_{(n-2 N-2) / 2}^{(1)}(k|x|)-\frac{n-2 N}{2 k|x|} H_{(n-2 N) / 2}^{(1)}(k|x|)\right)\right] \\
= & \frac{(-1)^{N} c_{n}}{2^{N} N ! k|x|^{(n-2 N) / 2}}\left[k^{(n-2 N) / 2}|x| H_{(n-2 N-2) / 2}^{(1)}(k|x|)\right] \\
= & \frac{(-1)^{N} c_{n} k^{(n-2 N-2) / 2}}{2^{N} N !|x|^{(n-2 N-2) / 2}} H_{(n-2 N-2) / 2}^{(1)}(k|x|) \\
= & \Phi_{k}^{(N+1)}(x), \quad \forall x \in \mathbb{R}^{n} \backslash\{0\},
\end{aligned}
$$

where (A.4) is used in the $3^{\text {rd }}$ equality above.

Lemma 5.3. For any $N \in \mathbb{N}, k \in(0, \infty)$, we have

$$
\left(\Delta+k^{2}\right) \Phi_{k}^{(N+1)}(x)=\Phi_{k}^{(N)}(x) \quad \text { pointwise at every } x \in \mathbb{R}^{n} \backslash\{0\}
$$


Proof. The proof proceeds by induction over $N$. For $N=1$, applying (5.16) yields

$$
\begin{aligned}
\left(\Delta+k^{2}\right) \Phi_{k}^{(2)}(x) & =\left(\Delta+k^{2}\right)\left[\frac{-1}{2 k} \frac{d}{d k} \Phi_{k}^{(1)}(x)\right] \\
& =\frac{-1}{2 k} \frac{d}{d k}\left(\Delta \Phi_{k}^{(1)}\right)(x)-\frac{k}{2} \frac{d}{d k} \Phi_{k}^{(1)}(x) \\
& =\frac{-1}{2 k} \frac{d}{d k}\left(\Delta \Phi_{k}^{(1)}\right)(x)+\Phi_{k}^{(1)}(x)-\frac{1}{2 k} \frac{d}{d k}\left[k^{2} \Phi_{k}^{(1)}(x)\right] \\
& =\Phi_{k}^{(1)}(x)-\frac{1}{2 k} \frac{d}{d k}\left[\left(\Delta+k^{2}\right) \Phi_{k}^{(1)}(x)\right] \\
& =\Phi_{k}^{(1)}(x), \quad \forall x \in \mathbb{R}^{n} \backslash\{0\} .
\end{aligned}
$$

For the last equality in (5.19) we used that $\Phi_{k}^{(1)} \equiv \Phi_{k}$, the fundamental solution of the Helmholtz operator (see item (2) in Theorem 4.1). This proves (5.18) when $N=1$.

Assume next that (5.18) holds for some $N \in \mathbb{N}$. Applying (5.16) and the induc- 
tive hypothesis yields

$$
\begin{aligned}
\left(\Delta+k^{2}\right) \Phi_{k}^{(N+2)}(x)= & \left(\Delta+k^{2}\right)\left[\frac{-1}{2(N+1) k} \frac{d}{d k} \Phi_{k}^{(N+1)}(x)\right] \\
= & \frac{-1}{2(N+1) k} \frac{d}{d k}\left(\Delta \Phi_{k}^{(N+1)}\right)(x)-\frac{k}{2(N+1)} \frac{d}{d k} \Phi_{k}^{(N+1)}(x) \\
= & \frac{-1}{2(N+1) k} \frac{d}{d k}\left(\Delta \Phi_{k}^{(N+1)}\right)(x)+\frac{1}{N+1} \Phi_{k}^{(N+1)}(x) \\
& -\frac{1}{2(N+1) k} \frac{d}{d k}\left[k^{2} \Phi_{k}^{(N+1)}(x)\right] \\
= & \frac{1}{N+1} \Phi_{k}^{(N+1)}(x)-\frac{1}{2(N+1) k} \frac{d}{d k}\left[\left(\Delta+k^{2}\right) \Phi_{k}^{(N+1)}(x)\right] \\
= & \frac{1}{N+1} \Phi_{k}^{(N+1)}(x)-\frac{1}{2(N+1) k} \frac{d}{d k} \Phi_{k}^{(N)}(x) \\
= & \frac{1}{N+1} \Phi_{k}^{(N+1)}(x)+\frac{1}{2(N+1) k} 2 N k \Phi_{k}^{(N+1)}(x) \\
= & \left(\frac{1}{N+1}+\frac{N}{N+1}\right) \Phi_{k}^{(N+1)}(x) \\
= & \Phi_{k}^{(N+1)}(x), \quad \forall x \in \mathbb{R}^{n} \backslash\{0\} .
\end{aligned}
$$

This completes the proof of (5.18).

The next lemma concerns the behavior of $\Phi_{k}^{(N)}$ near the origin.

Lemma 5.4. Let $N \in \mathbb{N}$ and $k \in(0, \infty)$. Then

$$
\lim _{x \rightarrow 0} \frac{\left|\Phi_{k}^{(N)}(x)\right|}{F(x)}=1
$$


where

$$
F(x)= \begin{cases}\frac{\Gamma((n-2 N) / 2)}{2^{2 N} \pi^{n / 2}(N-1) !} \frac{1}{|x|^{n-2 N}} \quad \text { if } \quad N<\frac{n}{2}, \\ \frac{1}{2^{n-1} \pi^{n / 2}(N-1) !}|\ln (k|x|)| & \text { if } \quad N=\frac{n}{2}, \quad \forall x \in \mathbb{R}^{n} \backslash\{0\} . \\ \frac{\Gamma((2 N-n) / 2)}{2^{n} \pi^{n / 2}(N-1) ! k^{2 N-n}} & \text { if } \quad N>\frac{n}{2} .\end{cases}
$$

Consequently, for each $R \in(0, \infty)$ there exists a finite constant $C=C(R, n, N, k)>0$ with the property that

$$
\left|\Phi_{k}^{(N)}(x)\right| \leq\left\{\begin{array}{lll}
C\left(1+\frac{1}{|x|^{n-2 N}}\right) & \text { if } & N<\frac{n}{2} \\
C(1+|\ln (|x|)|) & \text { if } & N=\frac{n}{2} \\
C & \text { if } & N>\frac{n}{2}
\end{array}\right.
$$

for every $x \in \mathbb{R}^{n}$ satisfying $0<|x| \leq R$. In particular,

$$
\Phi_{k}^{(N)} \text { is locally integrable in } \mathbb{R}^{n}
$$

Proof. To simplify the writing, we make use of the following notation: whenever two functions $f$ and $g$ are defined near the origin in $\mathbb{R}^{n}$ and satisfy $\lim _{x \rightarrow 0} \frac{f(x)}{g(x)}=1$, we will simply write $f \sim g$ as $x \rightarrow 0$.

To prove the desired estimates, we will make use of Lemma A.2. Note that since 
$\left|c_{n}\right|=1 /\left(2^{(n+2) / 2} \pi^{(n-2) / 2}\right)($ recall (4.1)), when $2 N=n$, formulas (5.1) and (A.10) yield

$$
\begin{aligned}
\left|\Phi_{k}^{(N)}(x)\right| & =\frac{\left|c_{n}\right|}{2^{N-1}(N-1) !}\left|H_{0}^{(1)}(k|x|)\right| \\
& \sim \frac{2}{\pi 2^{(n+2) / 2} \pi^{(n-2) / 2} 2^{N-1}(N-1) !}|\ln (k|x|)| \\
& =\frac{1}{2^{n-1} \pi^{n / 2}(N-1) !}|\ln (k|x|)| \quad \text { as } x \rightarrow 0 \text { in } \mathbb{R}^{n} .
\end{aligned}
$$

If $2 N \neq n$, we combine (5.1) with (A.11) to obtain

$$
\begin{aligned}
\left|\Phi_{k}^{(N)}(x)\right| & =\frac{\left|c_{n}\right| k^{n-2 N}}{2^{N-1}(N-1) !} \frac{\left|H_{(n-2 N) / 2}^{(1)}(k|x|)\right|}{(k|x|)^{(2-2 N) / 2}} \\
& \sim \frac{k^{n-2 N} 2^{|(n-2 N) / 2|} \Gamma(|(n-2 N) / 2|)}{\pi 2^{(n+2) / 2} \pi^{(n-2) / 2} 2^{N-1}(N-1) !} \frac{1}{(k|x|)^{(n-2 N) / 2+|(n-2 N) / 2|}} \\
& =\left\{\begin{array}{ll}
\frac{\Gamma((n-2 N) / 2)}{2^{2 N} \pi^{n / 2}(N-1) !} \frac{1}{|x|^{n-2 N}} & \text { if } \quad 2 N<n, \\
\frac{\Gamma((2 N-2) / 2)}{2^{n} \pi^{n / 2}(N-1) ! k^{2 N-n}} & \text { if } \quad 2 N>n,
\end{array} \quad \text { as } x \rightarrow 0 \text { in } \mathbb{R}^{n} .\right.
\end{aligned}
$$

Estimates (5.25) and (5.26) establish (5.21).

To proceed with the proof of (5.23), fix some $R \in(0, \infty)$, and define the positive functions

$$
g^{(N)}(r):=\left\{\begin{array}{lll}
1+\frac{1}{r^{n-2 N}} & \text { if } \quad N<\frac{n}{2}, \\
1+|\ln (r)| & \text { if } \quad N=\frac{n}{2}, \quad \forall r \in(0, \infty), \\
1 & \text { if } \quad N>\frac{n}{2},
\end{array}\right.
$$


and

$$
G^{(N)}(r):= \begin{cases}\frac{\Gamma((n-2 N) / 2)}{2^{2 N} \pi^{n / 2}(N-1) !} \frac{1}{r^{n-2 N}} & \text { if } \quad N<\frac{n}{2}, \\ \frac{1}{2^{n-1} \pi^{n / 2}(N-1) !}|\ln (k r)| & \text { if } \quad N=\frac{n}{2}, \quad \forall r \in(0, \infty) . \\ \frac{\Gamma((2 N-n) / 2)}{2^{n} \pi^{n / 2}(N-1) ! k^{2 N-n}} & \text { if } \quad N>\frac{n}{2},\end{cases}
$$

Recall $\phi_{k}^{(N)}$ from (5.2) and note that (5.21) implies $\lim _{r \rightarrow 0^{+}} \frac{\left|\phi_{k}^{(N)}(r)\right|}{G^{(N)}(r)}=1$. Thus, there exists $\delta \in(0, R)$ such that $r \in(0, \delta)$ implies $0<\frac{\left|\phi_{k}^{(N)}(r)\right|}{G^{(N)}(r)}<2$, and hence $\left|\phi_{k}^{(N)}(r)\right|<2 G^{(N)}(r)$ for $r \in(0, \delta)$.

Set $M:=\max _{r \in[\delta, R]}\left\{1+\left|\phi_{k}^{(N)}(r)\right|\right\}$. Since $g^{(N)}(r) \geq 1$ for each $r \in(0, \infty)$ we have the estimate $\left|\phi_{k}^{(N)}(r)\right|<M \leq M g^{(N)}(r)$ for $r \in[\delta, R]$. Turning our attention to $r \in(0, \delta)$, we approach each case separately.

For $N<\frac{n}{2}$, set $m:=\frac{\Gamma((n-2 N) / 2)}{2^{2 N-1} \pi^{n / 2}(N-1) !}$. Then for $r \in(0, \delta)$,

$$
\left|\phi_{k}^{(N)}(r)\right|<2 G^{(N)}(r)=\frac{m}{r^{n-2 N}}<m\left(1+\frac{1}{r^{n-2 N}}\right)=m g^{(N)}(r) .
$$

Thus setting $C:=\max \{m, M\}$ yields $\left|\Phi_{k}^{(N)}(x)\right|<C g^{(N)}(|x|)$ for $|x| \in(0, R]$.

$$
\begin{aligned}
& \text { For } N=\frac{n}{2}, r \in(0, \delta) \\
& \qquad \begin{aligned}
\left|\phi_{k}^{(N)}(r)\right| & <2 G^{(N)}(r)=\frac{1}{2^{n-2} \pi^{n / 2}(N-1) !}|\ln (k r)| \\
& \leq \frac{|\ln (k)|}{2^{n-2} \pi^{n / 2}(N-1) !}+\frac{1}{2^{n-2} \pi^{n / 2}(N-1) !}|\ln (r)| \\
& \leq m(1+|\ln (r)|)=m g^{(N)}(r),
\end{aligned}
\end{aligned}
$$


where

$$
m:=\max \left\{\frac{|\ln (k)|}{2^{n-2} \pi^{n / 2}(N-1) !}, \frac{1}{2^{n-2} \pi^{n / 2}(N-1) !}\right\} .
$$

Thus setting $C:=\max \{m, M\}$ yields $\left|\Phi_{k}^{(N)}(x)\right|<C g^{(N)}(|x|)$ for $|x| \in(0, R]$.

Finally, for $N>\frac{n}{2}$, set $m:=\frac{\Gamma((2 N-n) / 2)}{2^{n-1} \pi^{n / 2}(N-1) ! k^{2 N-n}}$. Then

$$
\left|\phi_{k}^{(N)}(r)\right|<2 G^{(N)}(r)=m=m g^{(N)}(r)
$$

for each $r \in(0, \delta)$. Consequently, by settings $C:=\max \{m, M\}$ the latter yields $\left|\Phi_{k}^{(N)}(x)\right|<C g^{(N)}(|x|)$ for $|x| \in(0, R]$.

Theorem 5.5. Suppose $n \in \mathbb{N}, n \geq 2$, and fix $k \in(0, \infty)$. Then for any $N \in \mathbb{N}$, the locally integrable function $\Phi_{k}^{(N)}$ defined in (5.1) is a fundamental solution of the iterated Helmholtz operator $\left(\Delta+k^{2}\right)^{N}$ in $\mathbb{R}^{n}$.

Proof. It is immediate that $\Phi_{k}^{(N)} \in \mathscr{C}^{\infty}\left(\mathbb{R}^{n} \backslash\{0\}\right)$ and we claim that

$$
\left(\Delta+k^{2}\right)^{N} \Phi_{k}^{(N)}(x)=0 \text { point-wise for every } x \in \mathbb{R}^{n} \backslash\{0\} .
$$

Indeed, by applying Lemma $5.3(N-1)$-times and then using (1) in Theorem 4.1 we obtain

$$
\left(\Delta+k^{2}\right)^{N} \Phi_{k}^{(N)}=\left(\Delta+k^{2}\right)^{(N-1)} \Phi_{k}^{(N-1)}=\ldots=\left(\Delta+k^{2}\right) \Phi_{k}^{(1)}=0
$$

pointwise in $\mathbb{R}^{n} \backslash\{0\}$. From (5.24) it follows that $\Phi_{k}^{(N)} \in \mathcal{D}^{\prime}\left(\mathbb{R}^{n}\right)$ for every $N$. Fix an arbitrary $\varphi \in \mathscr{C}_{0}^{\infty}\left(\mathbb{R}^{n}\right)$. To conclude that $\Phi_{k}^{(N)}$ is a fundamental solution for $\left(\Delta+k^{2}\right)^{N}$ in $\mathbb{R}^{n}$, there remains to prove

$$
\int_{\mathbb{R}^{n}} \Phi_{k}^{(N)}(x)\left[\left(\Delta+k^{2}\right)^{N} \varphi\right](x) d \mathscr{L}^{n}(x)=\varphi(0) .
$$


To justify this, proceed by induction on $N$. The case $N=1$ is a consequence of (2) in Theorem 4.1, since $\Phi_{k}^{(1)}=\Phi_{k}$. Assume (5.34) holds for some fixed $N \in \mathbb{N}$. Using Lebesgue's Dominated Convergence Theorem (bearing in mind that $\varphi$ is compactly supported), integrations by parts and (5.32), together with Lemma 5.3 and the inductive hypothesis, we get

$$
\begin{aligned}
& \int_{\mathbb{R}^{n}} \Phi_{k}^{(N+1)}(x)\left[\left(\Delta+k^{2}\right)^{(N+1)} \varphi\right](x) d \mathscr{L}^{n}(x) \\
& \quad=\lim _{\varepsilon \rightarrow 0^{+}} \int_{\mathbb{R}^{n} \backslash B(0, \varepsilon)} \Phi_{k}^{(N+1)}(x)\left[\left(\Delta+k^{2}\right)^{(N+1)} \varphi\right](x) d \mathscr{L}^{n}(x) \\
& \quad=\lim _{\varepsilon \rightarrow 0^{+}}\left\{\int_{\mathbb{R}^{n} \backslash B(0, \varepsilon)}\left[\left(\Delta+k^{2}\right) \Phi_{k}^{(N+1)}\right](x)\left[\left(\Delta+k^{2}\right)^{N} \varphi\right](x) d \mathscr{L}^{n}(x)+I_{\varepsilon}+I I_{\varepsilon}\right\} \\
& \quad=\lim _{\varepsilon \rightarrow 0^{+}}\left\{\int_{\mathbb{R}^{n} \backslash B(0, \varepsilon)} \Phi_{k}^{(N)}(x)\left[\left(\Delta+k^{2}\right)^{N} \varphi\right](x) d \mathscr{L}^{n}(x)+I_{\varepsilon}+I I_{\varepsilon}\right\} \\
& \quad=\varphi(0)+\lim _{\varepsilon \rightarrow 0^{+}} I_{\varepsilon}+\lim _{\varepsilon \rightarrow 0^{+}} I I_{\varepsilon}
\end{aligned}
$$

where

$$
\begin{aligned}
I_{\varepsilon} & :=-\int_{\partial B(0, \varepsilon)} \Phi_{k}^{(N+1)}(x)\left\langle\frac{x}{\varepsilon}, \nabla\left(\Delta+k^{2}\right)^{N} \varphi(x)\right\rangle d \mathscr{H}^{n-1}(x), \\
I I_{\varepsilon} & :=\int_{\partial B(0, \varepsilon)}\left\langle\frac{x}{\varepsilon}, \nabla \Phi_{k}^{(N+1)}(x)\right\rangle\left[\left(\Delta+k^{2}\right)^{N} \varphi\right](x) d \mathscr{H}^{n-1}(x) .
\end{aligned}
$$

For each $\varepsilon \in(0,1)$, recalling $\phi_{k}^{(N)}$ from (5.2) and employing $g^{(N)}$ defined in (5.27), 
Lemma 5.4 permits us to estimate

$$
\begin{aligned}
\left|I_{\varepsilon}\right| & \leq \int_{\partial B(0, \varepsilon)}\left|\Phi_{k}^{(N+1)}(x)\right|\left|\nabla\left(\Delta+k^{2}\right)^{N} \varphi(x)\right| d \mathscr{H}^{n-1}(x) \\
& \leq \int_{\partial B(0, \varepsilon)}\left|\phi_{k}^{(N+1)}(\varepsilon)\right|\left\|\nabla\left(\Delta+k^{2}\right)^{N} \varphi\right\|_{L^{\infty}(B(0,1))} d \mathscr{H}^{n-1}(x) \\
& =\omega_{n-1} \varepsilon^{n-1}\left|\phi_{k}^{(N+1)}(\varepsilon)\right|\left\|\nabla\left(\Delta+k^{2}\right)^{N} \varphi\right\|_{L^{\infty}(B(0,1))} \\
& \leq C_{1} \varepsilon^{n-1} g^{(N+1)}(\varepsilon),
\end{aligned}
$$

for some constant $C_{1}=C_{1}(n, N, k, \varphi)>0$ independent of $\varepsilon$. Since

$$
\varepsilon^{n-1} g^{(N+1)}(\varepsilon)= \begin{cases}\varepsilon^{n-1}+\varepsilon^{2 N+1} & \text { for } \quad N<\frac{n}{2}-1, \\ \varepsilon^{n-1}+\varepsilon^{n-1}|\ln (\varepsilon)| & \text { for } \quad N=\frac{n}{2}-1, \\ \varepsilon^{n-1} & \text { for } \quad N>\frac{n}{2}-1,\end{cases}
$$

and $n \geq 2$, we have

$$
\lim _{\varepsilon \rightarrow 0^{+}}\left|I_{\varepsilon}\right|=C_{1} \lim _{\varepsilon \rightarrow 0^{+}} \varepsilon^{n-1} g^{(N+1)}(\varepsilon)=0 .
$$

Employing (5.3), the treatment of $\left|I I_{\varepsilon}\right|$ is similar. For each $\varepsilon \in(0,1)$, Lemma 5.4 
permits us to estimate

$$
\begin{aligned}
\left|I I_{\varepsilon}\right| & \leq \int_{\partial B(0, \varepsilon)}\left|\nabla \Phi_{k}^{(N+1)}(x)\right|\left|\left(\Delta+k^{2}\right)^{N} \varphi(x)\right| d \mathscr{H}^{n-1}(x) \\
& \leq \int_{\partial B(0, \varepsilon)}\left|\left(\nabla \Phi_{k}^{(N+1)}\right)(x)\right|\left\|\left(\Delta+k^{2}\right)^{N} \varphi\right\|_{L^{\infty}(B(0,1))} d \mathscr{H}^{n-1}(x) \\
& =\left\|\left(\Delta+k^{2}\right)^{N} \varphi\right\|_{L^{\infty}(B(0,1))} \int_{\partial B(0, \varepsilon)}\left|\frac{x}{2 N} \Phi_{k}^{(N)}(x)\right| d \mathscr{H}^{n-1}(x) \\
& =\left\|\left(\Delta+k^{2}\right)^{N} \varphi\right\|_{L^{\infty}(B(0,1))} \frac{\varepsilon}{2 N}\left|\phi_{k}^{(N)}(\varepsilon)\right| \omega_{n-1} \varepsilon^{n-1} \\
& \leq C_{2} \varepsilon^{n} g^{(N)}(\varepsilon),
\end{aligned}
$$

for some constant $C_{2}=C_{2}(n, N, k, \varphi)>0$ independent of $\varepsilon$. Since

$$
\varepsilon^{n} g^{(N)}(\varepsilon)=\left\{\begin{array}{lll}
\varepsilon^{n}+\varepsilon^{2 N} & \text { for } & N<\frac{n}{2}, \\
\varepsilon^{n}+\varepsilon^{n}|\ln (\varepsilon)| & \text { for } & N=\frac{n}{2}, \\
\varepsilon^{n} & \text { for } & N>\frac{n}{2},
\end{array}\right.
$$

and $n \geq 2$, we have

$$
\lim _{\varepsilon \rightarrow 0^{+}}\left|I I_{\varepsilon}\right|=C_{2} \lim _{\varepsilon \rightarrow 0^{+}} \varepsilon^{n} g^{(N)}(\varepsilon)=0 .
$$

Finally, by (5.40) and (5.43), equation (5.35) becomes

$$
\int_{\mathbb{R}^{n}} \Phi_{k}^{(N+1)}(x)\left[\left(\Delta+k^{2}\right)^{(N+1)} \varphi\right](x) d \mathscr{L}^{n}(x)=\varphi(0),
$$

concluding the proof of (5.34), and with it finishing the proof of the theorem.

In the last part of this section we analyze the behavior of $\Phi_{k}^{(N)}$ at infinity. To 
simplify notation, we introduce the constants

$$
b_{n, k}^{(N)}:=\frac{k^{(n-2 N-1) / 2} e^{-i \pi(n+2 N-1) / 4}}{2^{(n+2 N-1) / 2} \pi^{(n-1) / 2}(N-1) !} \quad \text { for each } N \in \mathbb{N} .
$$

Recalling the constant $b_{n, k}$ from (4.3), we find that

$$
\begin{aligned}
b_{n, k}^{(N)} & =\frac{e^{-i \pi(N-1) / 2}}{2^{N-1} k^{N-1}(N-1) !} \frac{k^{(n-3) / 2} e^{-i \pi(n+1) / 4}}{2^{(n+1) / 2} \pi^{(n-1) / 2}} \\
& =\frac{b_{n, k}}{(2 i k)^{N-1}(N-1) !}
\end{aligned}
$$

for each $N \in \mathbb{N}$.

Lemma 5.6. Fix $k \in(0, \infty), N \in \mathbb{N}$, and recall $b_{n, k}^{(N)}$ from (5.45). Then

$$
\Phi_{k}^{(N)}(x-y)=b_{n, k}^{(N)} \frac{e^{i k|x|} e^{-i k\langle\widehat{x}, y\rangle}}{|x|^{(n-2 N+1) / 2}}+O\left(|x|^{-(n-2 N+3) / 2}\right) \quad \text { as }|x| \rightarrow \infty,
$$

uniformly for $y$ in compact subsets of $\mathbb{R}^{n}$, and

$$
\Phi_{k}^{(N)}(x-y)=b_{n, k}^{(N)} \frac{e^{i k|y|} e^{-i k\langle x, \widehat{y}\rangle}}{|y|^{(n-2 N+1) / 2}}+O\left(|y|^{-(n-2 N+3) / 2}\right) \quad \text { as }|y| \rightarrow \infty,
$$

uniformly for $x$ in compact subsets of $\mathbb{R}^{n}$.

Proof. First, a combination of (5.1) and (A.9) yields

$$
\begin{aligned}
\Phi_{k}^{(N)}(x) & =\frac{(-1)^{N-1} k^{(n-2 N) / 2}}{2^{(n+2 N) / 2} i \pi^{(n-2) / 2}(N-1) !|x|^{(n-2 N) / 2}}\left[\left(\frac{2}{\pi k|x|}\right)^{1 / 2} e^{i(k|x|-(n-2 N) \pi / 4-\pi / 4)}+O\left(|x|^{-3 / 2}\right)\right] \\
& =\frac{k^{(n-2 N-1) / 2} e^{-i \pi(N-1)} e^{-i \pi / 2} e^{-i \pi(n-2 N+1) / 4}}{2^{(n+2 N-1) / 2} \pi^{(n-1) / 2}(N-1) !} \frac{e^{i k|x|}}{|x|^{(n-2 N+1) / 2}}+O\left(|x|^{-(n-2 N+3) / 2}\right) \\
& =b_{n, k}^{(N)} \frac{e^{i k|x|}}{|x|^{(n-2 N+1) / 2}}+O\left(|x|^{-(n-2 N+3) / 2}\right) \quad \text { as }|x| \rightarrow \infty .
\end{aligned}
$$


Next let $K \subseteq \mathbb{R}^{n}$ be a compact set, fix an arbitrary $y \in K$, and consider $x \in \mathbb{R}^{n}$ with $|x|$ sufficiently large. In this case $|x-y|$ is proportional to $|x|$, hence for any fixed $\lambda \in \mathbb{R}$ we have

$$
O\left(|x-y|^{\lambda}\right)=O\left(|x|^{\lambda}\right) \text { as } x \rightarrow \infty \text { uniformly for } y \in K
$$

Moreover, the Mean Value Theorem gives

$$
\frac{1}{|x-y|^{\lambda}}=\frac{1}{|x|^{\lambda}}+O\left(|x|^{-(\lambda+1)}\right) \quad \text { as }|x| \rightarrow \infty, \quad \text { uniformly for } y \in K
$$

In addition, we claim that

$$
\frac{e^{i k|x-y|}}{|x-y|^{\lambda}}=\frac{e^{i k|x|} e^{-i k\langle\widehat{x}, y\rangle}}{|x|^{\lambda}}+O\left(|x|^{-(\lambda+1)}\right) \quad \text { as }|x| \rightarrow \infty, \quad \text { uniformly for } y \in K
$$

Indeed

$$
\frac{e^{i k|x-y|}}{|x-y|^{\lambda}}-\frac{e^{i k|x|} e^{-i k\langle\widehat{x}, y\rangle}}{|x|^{\lambda}}=I+I I
$$

where

$$
I:=\left(\frac{1}{|x-y|^{\lambda}}-\frac{1}{|x|^{\lambda}}\right) e^{i k|x-y|}
$$

and

$$
I I:=\left(1-e^{i k(|x|-\langle\widehat{x}, y\rangle-|x-y|)}\right) \frac{e^{i k|x-y|}}{|x|^{\lambda}}
$$

By (5.51) we see that

$$
I=O\left(|x|^{-(\lambda+1)}\right) \quad \text { as }|x| \rightarrow \infty, \quad \text { uniformly for } y \in K
$$


Hence (5.52) will follow once we show that

$$
\left|1-e^{i k(|x|-\langle\widehat{x}, y\rangle-|x-y|)}\right|=O\left(|x|^{-1}\right) \quad \text { as }|x| \rightarrow \infty, \quad \text { uniformly for } y \in K
$$

To this end, notice that

$$
\begin{aligned}
\left|1-e^{i a}\right|^{2} & =|1-\cos (a)-i \sin (a)|^{2}=(1-\cos (a)-i \sin (a))(1-\cos (a)+i \sin (a)) \\
& =(1-\cos (a))^{2}+\sin ^{2}(a)=1-2 \cos (a)+\cos ^{2}(a)+\sin ^{2}(a)=2-2 \cos (a) \\
& =4\left(\frac{1-\cos (a)}{2}\right)=4 \sin ^{2}\left(\frac{a}{2}\right) \leq 4\left(\frac{a}{2}\right)^{2}=a^{2} \quad \text { for all } a \in \mathbb{R} .
\end{aligned}
$$

Thus $\left|1-e^{i a}\right| \leq|a|$, so (5.57) will follow if we can show that

$$
|x|-\langle\widehat{x}, y\rangle-|x-y|=O\left(|x|^{-1}\right) \quad \text { as }|x| \rightarrow \infty, \quad \text { uniformly for } y \in K
$$

Recalling that the Taylor series expansion of $(1+t)^{1 / 2}$ around zero is $(1+t)^{1 / 2}=$ $1+\frac{t}{2}+O\left(t^{2}\right)$, we compute

$$
\begin{aligned}
|x-y| & =(\langle x-y, x-y\rangle)^{1 / 2}=\left(|x|^{2}-2\langle x, y\rangle+|y|^{2}\right)^{1 / 2} \\
& =|x|\left(1+\frac{|y|^{2}}{|x|^{2}}-2 \frac{\langle\widehat{x}, y\rangle}{|x|}\right)^{1 / 2} \\
& =|x|\left(1+\frac{1}{2}\left(\frac{|y|^{2}}{|x|^{2}}-2 \frac{\langle\widehat{x}, y\rangle}{|x|}\right)+O\left(\left|\frac{|y|^{2}}{|x|^{2}}-2 \frac{\left.\langle\widehat{x}, y\rangle\right|^{2}}{|x|}\right|^{2}\right)\right) \\
& =|x|\left(1-\frac{\langle\widehat{x}, y\rangle}{|x|}+O\left(|x|^{-2}\right)\right) \\
& =|x|-\langle\widehat{x}, y\rangle+O\left(|x|^{-1}\right) \quad \text { as }|x| \rightarrow \infty, \quad \text { uniformly for } y \in K,
\end{aligned}
$$

demonstrating (5.59) and concluding our proof of (5.52). 
Making use of (5.52) and (5.50) in connection with (5.49) allows us to write

$$
\begin{aligned}
\Phi_{k}^{(N)}(x-y) & =b_{n, k}^{(N)} \frac{e^{i k|x-y|}}{|x-y|^{(n-2 N+1) / 2}}+O\left(|x-y|^{-(n-2 N+3) / 2}\right) \\
& =b_{n, k}^{(N)}\left(\frac{e^{i k|x|} e^{-i k\langle\widehat{x}, y\rangle}}{|x|^{(n-2 N+1) / 2}}+O\left(|x|^{-(n-2 N+3) / 2}\right)\right)+O\left(|x|^{-(n-2 N+3) / 2}\right) \\
& =b_{n, k}^{(N)} \frac{e^{i k|x|} e^{-i k\langle\widehat{x}, y\rangle}}{|x|^{(n-2 N+1) / 2}}+O\left(|x|^{-(n-2 N+3) / 2}\right)
\end{aligned}
$$

as $|x| \rightarrow \infty$, uniformly for $y \in K$. This proves (5.47). In addition, since $\Phi_{k}^{(N)}$ is an even functions, the decay in (5.48) follows directly from (5.47).

Lemma 5.7. Fix $k \in(0, \infty), N \in \mathbb{N}$, and recall $b_{n, k}^{(N)}$ from (5.45). Then

$$
\begin{aligned}
\left(D_{k} \Phi_{k}^{(N)}\right)(x-y) & =\Phi_{k}^{(N)}(x-y)\left(i k \widehat{x}+k e_{n+1}\right)+O\left(|x|^{-(n-2 N+3) / 2}\right) \\
& =k b_{n, k}^{(N)} \frac{e^{i k|x|} e^{-i k(\widehat{x}, y\rangle}}{|x|^{(n-2 N+1) / 2}}\left(i \widehat{x}+e_{n+1}\right)+O\left(|x|^{-(n-2 N+3) / 2}\right)
\end{aligned}
$$

as $|x| \rightarrow \infty$ uniformly for $y$ in compact subsets of $\mathbb{R}^{n}$, and

$$
\begin{aligned}
\left(D_{k} \Phi_{k}^{(N)}\right)(x-y) & =\Phi_{k}^{(N)}(x-y)\left(-i k \widehat{y}+k e_{n+1}\right)+O\left(|y|^{-(n-2 N+3) / 2}\right) \\
& =k b_{n, k}^{(N)} \frac{e^{i k|y|} e^{-i k\langle x, \widehat{y}\rangle}}{|y|^{(n-2 N+1) / 2}}\left(-i \widehat{y}+e_{n+1}\right)+O\left(|y|^{-(n-2 N+3) / 2}\right)
\end{aligned}
$$

as $|y| \rightarrow \infty$ uniformly for $x$ in compact subsets of $\mathbb{R}^{n}$.

Proof. To begin, we claim that

$$
\left(D_{k} \Phi_{k}^{(N)}\right)(x)=k b_{n, k}^{(N)} \frac{e^{i k|x|}}{|x|^{(n-2 N+1) / 2}}\left(i \widehat{x}+e_{n+1}\right)+O\left(|x|^{-(n-2 N+3) / 2}\right) \quad \text { as }|x| \rightarrow \infty
$$


Recalling (5.5), for each $x \in \mathbb{R}^{n} \backslash\{0\}$, we write

$$
\left.\left(D_{k} \Phi_{k}^{(N)}\right)(x)=\frac{(-1)^{N} k^{(n-2 N+2) / 2}}{2^{(n+2 N) / 2} i \pi^{(n-2) / 2}(N-1) !}\left[I(x) \widehat{x}-I I(x) e_{n+1}\right)\right]
$$

where

$$
\begin{gathered}
I(x):=\frac{H_{(n-2 N+2) / 2}^{(1)}(k|x|)}{|x|^{(n-2 N) / 2}} \text { and } I I(x):=\frac{H_{(n-2 N) / 2}^{(1)}(k|x|)}{|x|^{(n-2 N) / 2}} \\
\text { for every } x \in \mathbb{R}^{n} \backslash\{0\} .
\end{gathered}
$$

Invoking (A.9) we obtain

$$
I(x)=\left(\frac{2}{\pi k}\right)^{1 / 2} e^{-i \pi(n-2 N+1) / 4} e^{-i \pi / 2} \frac{e^{i k|x|}}{|x|^{(n-2 N+1) / 2}}+O\left(|x|^{-(n-2 N+3) / 2}\right) \quad \text { as }|x| \rightarrow \infty
$$

and

$$
I I(x)=\left(\frac{2}{\pi k}\right)^{1 / 2} e^{-i \pi(n-2 N+1) / 4} \frac{e^{i k|x|}}{|x|^{(n-2 N+1) / 2}}+O\left(|x|^{-(n-2 N+3) / 2}\right) \quad \text { as }|x| \rightarrow \infty .
$$

From (5.65), (5.67), and (5.68), it follows that

$$
\begin{aligned}
\left(D_{k} \Phi_{k}^{(N)}\right)(x)= & \frac{(-1)^{N} k^{(n-2 N+2) / 2}}{2^{(n+2 N) / 2} i \pi^{(n-2) / 2}(N-1) !}\left(\frac{2}{\pi k}\right)^{1 / 2} e^{-i \pi(n-2 N+1) / 4} \frac{e^{i k|x|}}{|x|^{(n-2 N+1) / 2}}\left(-i \widehat{x}-e_{n+1}\right) \\
& +O\left(|x|^{-(n-2 N+3) / 2}\right) \\
= & \frac{k^{(n-2 N+1) / 2} e^{-i \pi(n+2 N-1) / 4}}{2^{(n+2 N-1) / 2} \pi^{(n-1) / 2}(N-1) !} \frac{e^{i k|x|}}{|x|^{(n-2 N+1) / 2}}\left(i \widehat{x}+e_{n+1}\right) \\
& +O\left(|x|^{-(n-2 N+3) / 2}\right) \\
= & k b_{n, k}^{(N)} \frac{e^{i k|x|}}{|x|^{(n-2 N+1) / 2}}\left(i \widehat{x}+e_{n+1}\right)+O\left(|x|^{-(n-2 N+3) / 2}\right) \quad \text { as }|x| \rightarrow \infty, \quad(5.69)
\end{aligned}
$$

completing the proof of (5.64)

Next let $K \subseteq \mathbb{R}^{n}$ be compact, $y \in K$, and $x \in \mathbb{R}^{n}$ with $|x|$ sufficiently large. Then 
$|x-y|$ is proportional to $|x|$, hence (5.50) holds for any fixed $\lambda \in \mathbb{R}$. Moreover, the Mean Value Theorem gives

$$
\frac{x-y}{|x-y|}=\frac{x}{|x|}+O\left(|x|^{-1}\right) \quad \text { as }|x| \rightarrow \infty, \quad \text { uniformly for } y \in K
$$

To prove (5.62), we write (5.64) with $x$ replaced by $x-y$, then use (5.70), (5.52), and (5.50) to write

$$
\begin{aligned}
\left(D_{k} \Phi_{k}^{(N)}\right)(x-y) & =k b_{n, k}^{(N)} \frac{e^{i k|x-y|}}{|x-y|^{(n-2 N+1) / 2}}\left(i \widehat{x-y}+e_{n+1}\right)+O\left(|x-y|^{-(n-2 N+3) / 2}\right) \\
& =k b_{n, k}^{(N)} \frac{e^{i k|x|} e^{-i k\langle\widehat{x}, y\rangle}}{|x|^{(n-2 N+1) / 2}}\left(i \widehat{x}+e_{n+1}\right)+O\left(|x|^{-(n-2 N+3) / 2}\right)
\end{aligned}
$$

as $|x| \rightarrow \infty$, uniformly for $y \in K$.

Finally, to prove (5.63) we reverse the roles of $x$ and $y$, setting $x \in K$ and taking $|y|$ arbitrarily large. In this case, (5.64) gives us

$$
\begin{gathered}
\left(D_{k} \Phi_{k}^{(N)}\right)(x-y)=k b_{n, k}^{(N)} \frac{e^{i k|x-y|}}{|x-y|^{(n-2 N+1) / 2}}\left(i \widehat{x-y}+e_{n+1}\right)+O\left(|x-y|^{-(n-2 N+3) / 2}\right) \\
=k b_{n, k}^{(N)} \frac{e^{i k|y-x|}}{|y-x|^{(n-2 N+1) / 2}}\left(-i \widehat{y-x}+e_{n+1}\right)+O\left(|y-x|^{-(n-2 N+3) / 2}\right)
\end{gathered}
$$

as $|y| \rightarrow \infty$. Consequently, (5.63) will follow from (5.72) by employing the same type of reasoning as in the proof of the last equality in (5.71). This finishes the proof of the lemma. 


\section{Fundamental solutions for iterated perturbed Dirac operators}

Throughout this section we consider $n \in \mathbb{N}, n \geq 2$, fixed. Also let $k \in(0, \infty)$ be fixed. Recall that the perturbed Dirac operators $D_{ \pm k}:=D \pm k e_{n+1}$ from (2.24) are "square roots" of the Helmholtz operator $\Delta+k^{2}=-D_{k}^{2}=-D_{-k}^{2}$ (see (2.27)). This suggests defining for each $N \in \mathbb{N}$ the $C \ell_{n+1}$-valued functions (recall $\Phi_{k}^{(N)}$ from (5.1)):

$$
\Theta_{k}^{(N)}(x):=\left\{\begin{array}{ll}
(-1)^{N / 2} \Phi_{k}^{(N / 2)}(x) & \text { if } N \text { is even, } \\
(-1)^{(N+1) / 2} D_{k} \Phi_{k}^{((N+1) / 2)}(x) & \text { if } N \text { is odd, }
\end{array} \text { for all } x \in \mathbb{R}^{n} \backslash\{0\},\right.
$$

and

$$
\Theta_{-k}^{(N)}(x):=\left\{\begin{array}{ll}
(-1)^{N / 2} \Phi_{k}^{(N / 2)}(x) & \text { if } N \text { is even, } \\
(-1)^{(N+1) / 2} D_{-k} \Phi_{k}^{(N+1) / 2)}(x) & \text { if } N \text { is odd, }
\end{array} \text { for all } x \in \mathbb{R}^{n} \backslash\{0\} .\right.
$$

Our first lemma expresses $\Theta_{k}^{(N)}$ and $\Theta_{-k}^{(N)}$ in terms of Hankel functions.

Lemma 6.1. Let $k \in(0, \infty)$ be fixed and recall the constant $c_{n}$ from (4.2). For each $N \in \mathbb{N}$ and every $x \in \mathbb{R}^{n} \backslash\{0\}$, the following formulas hold:

$$
\begin{gathered}
\Theta_{k}^{(N)}(x)=\frac{-c_{n} k^{(n-N) / 2}}{2^{(N-2) / 2}\left(\frac{N-2}{2}\right) !|x|^{(n-N) / 2}} H_{(n-N) / 2}^{(1)}(k|x|) \quad \text { if } N \text { is even, } \\
\Theta_{k}^{(N)}(x)=\frac{c_{n} k^{(n-N+1) / 2}}{2^{(N-1) / 2}\left(\frac{N-1}{2}\right) !|x|^{(n-N-1) / 2}}\left[H_{(n-N+1) / 2}^{(1)}(k|x|) \widehat{x}-H_{(n-N-1) / 2}^{(1)}(k|x|) e_{n+1}\right] \\
\text { if } N \text { is odd, }
\end{gathered}
$$


and

$$
\begin{gathered}
\Theta_{-k}^{(N)}(x)=\frac{-c_{n} k^{(n-N) / 2}}{2^{(N-2) / 2}\left(\frac{N-2}{2}\right) !|x|^{(n-N) / 2}} H_{(n-N) / 2}^{(1)}(k|x|) \quad \text { if } N \text { is even, } \\
\Theta_{-k}^{(N)}(x)=\frac{c_{n} k^{(n-N+1) / 2}}{2^{(N-1) / 2}\left(\frac{N-1}{2}\right) !|x|^{(n-N-1) / 2}}\left[H_{(n-N+1) / 2}^{(1)}(k|x|) \widehat{x}+H_{(n-N-1) / 2}^{(1)}(k|x|) e_{n+1}\right]
\end{gathered}
$$

if $N$ is odd.

In particular,

$$
\Theta_{-k}^{(N)}(x)=(-1)^{N} \Theta_{k}^{(N)}(-x)
$$

Proof. The formula in (6.3) follows by combining (6.1) with (5.1), while formula (6.4) results from combining (6.1) with (5.5). Likewise, (6.5)-(6.6) follow from (6.2), (5.1), and (5.6). Finally, (6.7) is an immediate consequence of (6.3) and (6.5).

Lemma 6.2. Fix $N \in \mathbb{N}$ and $k \in(0, \infty)$. Then

$$
\Theta_{ \pm k}^{(N)}(x)= \begin{cases}O\left(|x|^{N-n}\right) & \text { if } \quad N<n, \\ O(\ln |x|) & \text { if } \quad N=n, \quad \text { as } x \rightarrow 0 . \\ O(1) & \text { if } \quad N>n,\end{cases}
$$

Corresponding to $N=n$ and $n$ being odd, the stronger result $\left|\Theta_{ \pm k}^{(n)}(x)\right|=O(1)$ as $x \rightarrow 0$ holds.

Proof. From (2.6), (6.3) and (6.5), for every $x \in \mathbb{R}^{n} \backslash\{0\}$, we obtain

$$
\left|\Theta_{ \pm k}^{(N)}(x)\right|=\frac{k^{(n-N) / 2}}{2^{(N+n) / 2} \pi^{(n-2) / 2}\left(\frac{N-2}{2}\right) !|x|^{(n-N) / 2}}\left|H_{(n-N) / 2}^{(1)}(k|x|)\right|
$$


if $N$ is even, and

$$
\begin{aligned}
\left|\Theta_{ \pm k}^{(N)}(x)\right|=\frac{k^{(n-N+1) / 2}}{2^{(N+n+1) / 2} \pi^{(n-2) / 2}\left(\frac{N-1}{2}\right) !|x|^{(n-N-1) / 2}} \times \\
\quad \times \sqrt{\left|H_{(n-N+1) / 2}^{(1)}(k|x|)\right|^{2}+\left|H_{(n-N-1) / 2}^{(1)}(k|x|)\right|^{2}}
\end{aligned}
$$

if $N$ is odd. Observe that (A.10) implies

$$
H_{\lambda}^{(1)}(k r)=\left\{\begin{array}{ll}
O(\ln (r)) & \text { if } \quad \lambda=0, \\
O\left(r^{-|\lambda|}\right) & \text { if } \quad \lambda \neq 0,
\end{array} \quad \text { as } r \rightarrow 0^{+} .\right.
$$

This combined with (6.10) yields (6.8) when $N$ is even.

Proceeding under the assumption $N$ is odd, write $\left|\Theta_{ \pm k}^{(N)}(x)\right|^{2}=I(x)+I I(x)$, where

$$
I(x):=\frac{C^{2}}{|x|^{n-N-1}}\left|H_{(n-N+1) / 2}^{(1)}(k|x|)\right|^{2} \quad \text { and } \quad I I(x):=\frac{C^{2}}{|x|^{n-N-1}}\left|H_{(n-N-1) / 2}^{(1)}(k|x|)\right|^{2},
$$

with

$$
C:=\frac{k^{(n-N+1) / 2}}{2^{(N+n+1) / 2} \pi^{(n-2) / 2}\left(\frac{N-1}{2}\right) !} .
$$


Now (6.11) and (6.12) yield

$$
\begin{aligned}
& I(x)=\left\{\begin{array}{llc}
O\left(|x|^{-n+N+1}(\ln |x|)^{2}\right) & \text { if } & (n-N+1) / 2=0, \\
O\left(|x|^{-n+N+1-|n-N+1|}\right) & \text { if } \quad & (n-N+1) / 2 \neq 0
\end{array}\right.
\end{aligned}
$$

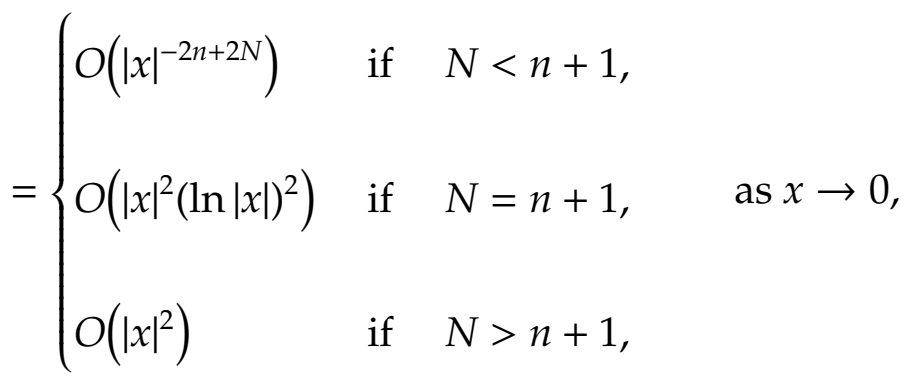

and

$$
\begin{aligned}
& I I(x)=\left\{\begin{array}{llc}
O\left(|x|^{-n+N+1}(\ln |x|)^{2}\right) & \text { if } & (n-N-1) / 2=0, \\
O\left(|x|^{-n+N+1-|n-N-1|}\right) & \text { if } & (n-N-1) / 2 \neq 0,
\end{array}\right. \\
& =\left\{\begin{array}{lll}
O\left(|x|^{-2 n+2 N+2}\right) & \text { if } \quad N<n-1, \\
O\left((\ln |x|)^{2}\right) & \text { if } \quad N=n-1, \quad \text { as } x \rightarrow 0 . \\
O(1) & \text { if } \quad N>n-1,
\end{array}\right.
\end{aligned}
$$


By (6.14) and (6.15) we have

$$
\Theta_{ \pm k}^{(N)}(x)= \begin{cases}O\left(|x|^{-n+N}\right)+O\left(|x|^{-n+N+1}\right) & \text { if } \quad N<n-1, \\
O\left(|x|^{-1}\right)+O(\ln |x|) & \text { if } \quad N=n-1, \\
O(1)+O(1) & \text { if } \quad N=n, \\
O(|x| \ln |x|)+O(1) & \text { if } \quad N=n+1, \\
O(|x|)+O(1) & \text { if } \quad N>n+1 . \\
= & \begin{array}{ll}
O\left(|x|^{N-n}\right) \quad \text { if } \quad N<n, \\
O(1) & \text { if } \quad N \geq n,
\end{array}\end{cases}
$$

from which (6.8) follows a fortiori.

Corollary 6.3. Fix $N \in \mathbb{N}$ and $k \in(0, \infty)$. Then $\Theta_{k}^{(N)}$ and $\Theta_{-k}^{(N)}$ are locally integrable in $\mathbb{R}^{n}$, and there holds

$$
\begin{gathered}
\Theta_{ \pm k}^{(N)}(x)=o\left(|x|^{1-n}\right) \text { as } x \rightarrow 0 \text { if } N \geq 2, \\
\text { and } \Theta_{ \pm k}^{(1)}(x)=O\left(|x|^{1-n}\right) \text { as } x \rightarrow 0 .
\end{gathered}
$$

Proof. Suppose first that $N \geq 2$. Making use of (6.8), there exists some constant $C \in(0, \infty)$ independent of $x$ for which we may write

$$
\frac{\left|\Theta_{ \pm k}^{(N)}(x)\right|}{|x|^{1-n}}=\frac{\left|\Theta_{ \pm k}^{(N)}(x)\right|}{|x|^{N-n}}|x|^{N-1} \leq C|x|^{N-1} \quad \text { as } x \rightarrow 0 \quad \text { if } \quad N<n
$$




$$
\frac{\left|\Theta_{ \pm k}^{(N)}(x)\right|}{|x|^{1-n}}=\frac{\left|\Theta_{ \pm k}^{(N)}(x)\right|}{|\ln | x||} \cdot \frac{|\ln | x||}{|x|^{1-n}} \leq C \frac{|\ln | x||}{|x|^{1-n}} \quad \text { as } x \rightarrow 0 \quad \text { if } \quad N=n
$$

and

$$
\frac{\left|\Theta_{ \pm k}^{(N)}(x)\right|}{|x|^{1-n}} \leq C|x|^{n-1} \quad \text { as } x \rightarrow 0 \quad \text { if } \quad N>n
$$

Now the statement in the first line of (6.17) follows from (6.18)-(6.20) (bearing in mind that $n \geq 2$ as well). As for the statement in the second line of (6.17), this corresponds to $N=1$, hence $N<n$, and is contained in (6.8). It remains to show that $\Theta_{k}^{(N)}$ and $\Theta_{-k}^{(N)}$ are locally integrable in $\mathbb{R}^{n}$.

Clearly from (6.17) we have $\Theta_{ \pm k}^{(N)}(x)=O\left(|x|^{1-n}\right)$ as $x \rightarrow 0$. In particular, if $\rho \in(0,1)$ is fixed, there exists some constant $C \in(0, \infty)$ so that $\left|\Theta_{ \pm k}^{(N)}(x)\right| \leq C|x|^{1-n}$, for all $x \in B(0, \rho)$. Then for any compact set $A \subseteq \mathbb{R}^{n}$, we have

$$
\begin{aligned}
\int_{A}\left|\Theta_{ \pm k}^{(N)}(x)\right| d \mathscr{L}^{n}(x) & \leq \int_{A \backslash B(0, \rho)}\left|\Theta_{ \pm k}^{(N)}(x)\right| d \mathscr{L}^{n}(x)+\int_{B(0, \rho)}\left|\Theta_{ \pm k}^{(N)}(x)\right| d \mathscr{L}^{n}(x) \\
& \leq \int_{A \backslash B(0, \rho)}\left|\Theta_{ \pm k}^{(N)}(x)\right| d \mathscr{L}^{n}(x)+C \int_{B(0, \rho)}|x|^{1-n} d \mathscr{L}^{n}(x) .
\end{aligned}
$$

Since $\Theta_{ \pm k}^{(N)} \in \mathscr{C}^{\infty}\left(\mathbb{R}^{n} \backslash\{0\}, C \ell_{n+1}\right)$ and $A \backslash B(0, \rho) \subseteq \mathbb{R}^{n} \backslash\{0\}$ is compact, it follows that

$$
\int_{A \backslash B(0, \rho)}\left|\Theta_{ \pm k}^{(N)}(x)\right| d \mathscr{L}^{n}(x)<+\infty
$$

Also,

$$
\begin{aligned}
\int_{B(0, \rho)}|x|^{1-n} d \mathscr{L}^{n}(x) & =\lim _{\varepsilon \rightarrow 0^{+}} \int_{\varepsilon}^{\rho} \int_{\partial B(0, r)}|x|^{1-n} d \mathscr{H}^{n-1}(x) d r \\
& =\lim _{\varepsilon \rightarrow 0^{+}} \int_{\varepsilon}^{\rho} \omega_{n-1} r^{n-1} r^{1-n} d r=\omega_{n-1} \rho<+\infty .
\end{aligned}
$$


In concert, (6.21), (6.22), and (6.23) imply $\Theta_{ \pm k}^{(N)} \in L_{l o c}^{1}\left(\mathbb{R}^{n}, C \ell_{n+1}\right)$, as desired.

Proposition 6.4. For any $N \in \mathbb{N}, k \in(0, \infty)$, we have the following recursive properties:

$$
D_{k} \Theta_{k}^{(N+1)}=\Theta_{k}^{(N)} \quad \text { in } \quad \mathcal{D}^{\prime}\left(\mathbb{R}^{n}, C \ell_{n+1}\right)
$$

and

$$
D_{-k} \Theta_{-k}^{(N+1)}=\Theta_{-k}^{(N)} \quad \text { in } \quad \mathcal{D}^{\prime}\left(\mathbb{R}^{n}, C \ell_{n+1}\right)
$$

Proof. Fix $k \in(0, \infty)$. From (6.3) and (6.5) it is clear that $\Theta_{ \pm k}^{(N)} \in \mathscr{C}^{1}\left(\mathbb{R}^{n} \backslash\{0\}, C \ell_{m}\right)$ for every $N \in \mathbb{N}$. The strategy for proving (6.24) and (6.25) is to invoke Proposition 3.1 with $P=D_{ \pm k}$.

To this end, fix $N \in \mathbb{N}$, and we first make the claim that

$$
D_{k} \Theta_{k}^{(N+1)}(x)=\Theta_{k}^{(N)}(x) \quad \text { for every } x \in \mathbb{R}^{n} \backslash\{0\}
$$

and

$$
D_{-k} \Theta_{-k}^{(N+1)}(x)=\Theta_{-k}^{(N)}(x) \quad \text { for every } x \in \mathbb{R}^{n} \backslash\{0\}
$$

When $N$ is odd, formulas (6.26)-(6.27) follow from (6.1) and (6.2) since (keeping in mind that $N+1$ is even)

$$
D_{ \pm k} \Theta_{ \pm k}^{(N+1)}(x)=(-1)^{(N+1) / 2} D_{ \pm k} \Phi_{k}^{((N+1) / 2)}(x)=\Theta_{ \pm k}^{(N)}(x), \quad \forall x \in \mathbb{R}^{n} \backslash\{0\}
$$

When $N$ is even, starting from (6.1) and (6.2) (used for the odd number $N+1$ ), then 
recalling (2.27), and then employing (5.18), and then again (6.1)-(6.2), we have

$$
\begin{aligned}
D_{ \pm k} \Theta_{ \pm k}^{(N+1)}(x) & =(-1)^{(\mathrm{N}+2) / 2} D_{ \pm k}^{2} \Phi_{k}^{((N+2) / 2)}(x) \\
& =(-1)^{N / 2}\left(\Delta+k^{2}\right) \Phi_{k}^{(N / 2+1)}(x) \\
& =(-1)^{N / 2} \Phi_{k}^{(N / 2)}(x)=\Theta_{ \pm k}^{(N)}(x), \quad \forall x \in \mathbb{R}^{n} \backslash\{0\} .
\end{aligned}
$$

Thus, (6.26)-(6.27) hold.

Moving on with verifying the hypothesis in Proposition 3.1 for $P:=D_{ \pm k}, u:=$ $\Theta_{ \pm k}^{(N+1)}$ and $v:=\Theta_{ \pm k}^{(N)}$, we first note that as a consequence of Corollary 6.3 we have $\Theta_{ \pm k}^{(N)} \in L_{l o c}^{1}\left(\mathbb{R}^{n}, C \ell_{n+1}\right)$ and $\Theta_{ \pm k}^{(N+1)}(x)=o\left(|x|^{1-n}\right)$ as $x \rightarrow 0$. At this point, in order to apply Proposition 3.1, there remains to check $D \Theta_{ \pm k}^{(N+1)}(x)=O\left(|x|^{1-n}\right)$ as $x \rightarrow 0$. The latter is a consequence of (6.26)-(6.27) and (6.17). Hence, Proposition 3.1 applies in the current setting and yields (6.24) and (6.25).

Theorem 6.5. Suppose $n \in \mathbb{N}, n \geq 2$, and fix $k \in(0, \infty)$. Then for each $N \in \mathbb{N}$, the locally integrable $C \ell_{n+1}$-valued function $\Theta_{k}^{(N)}$ defined in (6.3) satisfies the pointwise equation

$$
D_{k}^{N} \Theta_{k}^{(N)}(x)=0 \quad \text { for every } x \in \mathbb{R}^{n} \backslash\{0\},
$$

and is a fundamental solution of the operator $D_{k}^{N}$ in $\mathbb{R}^{n}$, i.e.,

$$
D_{k}^{N} \Theta_{k}^{(N)}=\delta, \quad \text { in } \quad \mathcal{D}^{\prime}\left(\mathbb{R}^{n}, C \ell_{n+1}\right)
$$

Likewise, for each $N \in \mathbb{N}$, the locally integrable $C \ell_{n+1}$-valued function $\Theta_{-k}^{(\mathrm{N})}$ defined in (6.5) satisfies the pointwise equation

$$
D_{-k}^{N} \Theta_{-k}^{(N)}(x)=0 \quad \text { for every } x \in \mathbb{R}^{n} \backslash\{0\},
$$


and is a fundamental solution of the operator $D_{-k}^{N}$ in $\mathbb{R}^{n}$, i.e.,

$$
D_{-k}^{N} \Theta_{-k}^{(N)}=\delta, \quad \text { in } \quad \mathcal{D}^{\prime}\left(\mathbb{R}^{n}, C \ell_{n+1}\right)
$$

Proof. Staring with (6.1) and (6.2) for $N=1$, then using (2.27), and then applying item (1) in Theorem 4.1, we compute

$$
D_{ \pm k} \Theta_{ \pm k}^{(1)}=-D_{ \pm k} D_{ \pm k} \Phi_{k}^{(1)}=\left(\Delta+k^{2}\right) \Phi_{k}=0 \quad \text { pointwise in } \quad \mathbb{R}^{n} \backslash\{0\}
$$

This and repeated applications of (6.26) or (6.27) now yield (6.30) and (6.32).

To prove (6.31) and (6.33) when $N=1$, we invoke Proposition 6.4 (with $N=1$ ), (2.27), (6.1)-(6.2) with $N=1$, and item (2) in Theorem 4.1, to write

$$
D_{ \pm k} \Theta_{ \pm k}^{(1)}=D_{ \pm k} D_{ \pm k} \Theta_{ \pm k}^{(2)}=-\left(\Delta+k^{2}\right) \Theta_{ \pm k}^{(2)}=\left(\Delta+k^{2}\right) \Phi_{k}=\delta, \quad \text { in } \quad \mathcal{D}^{\prime}\left(\mathbb{R}^{n}, C l_{n+1}\right)
$$

Corresponding to $N>1$, we apply $(N-1)$ times Proposition 6.4 and then use (6.35) to obtain

$$
D_{ \pm k}^{N} \Theta_{ \pm k}^{(N)}=D_{ \pm k}^{N-1} \Theta_{ \pm k}^{(N-1)}=\ldots=D_{ \pm k} \Theta_{ \pm k}^{(1)}=\delta, \quad \text { in } \quad \mathcal{D}^{\prime}\left(\mathbb{R}^{n}, C l_{n+1}\right)
$$

Thus, (6.31) and (6.33) are proved.

We conclude this section considering the behavior of $\Theta_{k}^{(N)}$ and $\Theta_{-k}^{(N)}$ at infinity. 
Lemma 6.6. Fix $k \in(0, \infty), N \in \mathbb{N}$, and recall the constant $b_{n, k}^{(N)}$ from (5.45). Then

$$
\begin{gathered}
\Theta_{ \pm k}^{(N)}(x-y)=(-1)^{N / 2} b_{n, k}^{(N / 2)} \frac{e^{i k|x|} e^{-i k\langle\widehat{x}, y\rangle}}{|x|^{(n-N+1) / 2}}+O\left(|x|^{-(n-N+3) / 2}\right) \\
\text { as }|x| \rightarrow \infty \text {, uniformly for } y \text { in compact subsets of } \mathbb{R}^{n} \\
\text { if } N \text { is even, }
\end{gathered}
$$

and

$$
\begin{gathered}
\Theta_{ \pm k}^{(N)}(x-y)=(-1)^{(N+1) / 2} k b_{n, k}^{((N+1) / 2)} \frac{e^{i k|x|} e^{-i k\langle\widehat{x}, y\rangle}}{|x|^{(n-N) / 2}}\left(i \widehat{x} \pm e_{n+1}\right)+O\left(|x|^{-(n-N+2) / 2}\right) \\
\text { as }|x| \rightarrow \infty \text {, uniformly for } y \text { in compact subsets of } \mathbb{R}^{n}, \\
\text { if } N \text { is odd, }
\end{gathered}
$$

Moreover,

$$
\begin{gathered}
\Theta_{ \pm k}^{(N)}(x-y)=(-1)^{N / 2} b_{n, k}^{(N / 2)} \frac{e^{i k|y|} e^{-i k\langle x, \widehat{y}\rangle}}{|y|^{(n-N+1) / 2}}+O\left(|y|^{-(n-N+3) / 2}\right) \\
\text { as }|y| \rightarrow \infty \text {, uniformly for } x \text { in compact subsets of } \mathbb{R}^{n}, \\
\text { if } N \text { is even, }
\end{gathered}
$$

and

$$
\begin{gathered}
\Theta_{ \pm k}^{(N)}(x-y)=(-1)^{(N+1) / 2} k b_{n, k}^{((N+1) / 2)} \frac{e^{i k|y|} e^{-i k\langle x, \widehat{y}\rangle}}{|y|^{(n-N) / 2}}\left(-i \widehat{y} \pm e_{n+1}\right)+O\left(|y|^{-(n-N+2) / 2}\right) \\
\text { as }|y| \rightarrow \infty, \text { uniformly for } x \text { in compact subsets of } \mathbb{R}^{n},
\end{gathered}
$$

if $N$ is odd.

Proof. Based on (6.1) and (6.2), the formulas in (6.37) and (6.39) follow immediately from Lemma 5.6. The formulas involving $\Theta_{+k}^{(N)}$ in (6.38) and (6.40) follow immediately from Lemma 5.7. For the formulas in (6.38) and (6.40) involving $\Theta_{-k}^{(N)}$ adapt 
the proof of Lemma 5.7 by replacing $e_{n+1}$ with $-e_{n+1}$ throughout, and the desired results are obtained. 


\section{Radiation conditions and integral representations for null-solutions of iterated perturbed Dirac opera- tors}

To state the next result recall the locally integrable functions $\Theta_{-k}^{(N)}$ defined in (6.5).

Lemma 7.1. Fix $k \in(0, \infty), N \in \mathbb{N}$, and suppose $\Omega$ is an open bounded subset of $\mathbb{R}^{n}$ with Lipschitz boundary $\partial \Omega$ and outward unit normal $v$. Consider an arbitrary $C \ell_{m}$-valued function $u \in \mathscr{C}^{N}\left(\bar{\Omega}, C \ell_{m}\right)$. Then for each $M \in\{1,2, \ldots, N\}$ and every every $x \in \Omega$, one has

$$
\begin{aligned}
\int_{\Omega \backslash B(x, \varepsilon)}( & \left.D_{-k}^{M} \Theta_{-k}^{(N)}\right)(y-x) \odot u(y) d \mathscr{L}^{n}(y) \\
= & \sum_{j=0}^{M-1}(-1)^{j} \int_{\partial \Omega} \Theta_{-k}^{(N-M+j+1)}(y-x) \odot v(y) \odot D_{k}^{j} u(y) d \mathscr{H}^{n-1}(y) \\
& -\sum_{j=0}^{M-1}(-1)^{j} \int_{\partial B(x, \varepsilon)} \Theta_{-k}^{(N-M+j+1)}(y-x) \odot \frac{y-x}{\varepsilon} \odot D_{k}^{j} u(y) d \mathscr{H}^{n-1}(y) \\
& +(-1)^{M} \int_{\Omega \backslash B(x, \varepsilon)} \Theta_{-k}^{(N)}(y-x) \odot D_{k}^{M} u(y) d \mathscr{L}^{n}(y)
\end{aligned}
$$

for every $\varepsilon \in\left(0, \frac{1}{2} \operatorname{dist}(x, \partial \Omega)\right)$.

Proof. To start, observe that a combination of (6.2), (2.27), and the fact that $\Phi_{k}^{(N)}$ is a scalar function for each $N \in \mathbb{N}$ (recall (5.1)) yields

$$
\Theta_{-k}^{(N)} D_{-k}=D_{-k} \Theta_{-k}^{(N)} \quad \text { in } \mathbb{R}^{n} \backslash\{0\}
$$

Fix $x \in \Omega$ and choose some arbitrary $\varepsilon \in\left(0, \frac{1}{2} \operatorname{dist}(x, \partial \Omega)\right)$. For simplicity of notation, set $\Omega_{\varepsilon}:=\Omega \backslash \overline{B(x, \varepsilon)}$. Thus, $\partial \Omega_{\varepsilon}=\partial \Omega \cup \partial B(x, \varepsilon)$ and if we continue to use $v$ to denote 
the outward unit normal to $\Omega_{\varepsilon}$, then

$$
v(y)=-\frac{y-x}{\varepsilon} \quad \text { for every } y \in \partial B(x, \varepsilon)
$$

In view of this notation, (7.1) rewrites as

$$
\begin{aligned}
\int_{\Omega_{\varepsilon}}\left(D_{-k}^{M} \Theta_{-k}^{(N)}\right)(y-x) \odot u(y) d \mathscr{L}^{n}(y) \\
=\sum_{j=0}^{M-1}(-1)^{j} \int_{\partial \Omega_{\varepsilon}} \Theta_{-k}^{(N-M+j+1)}(y-x) \odot v(y) \odot D_{k}^{j} u(y) d \mathscr{H}^{n-1}(y) \\
\quad+(-1)^{M} \int_{\Omega_{\varepsilon}} \Theta_{-k}^{(N)}(y-x) \odot D_{k}^{M} u(y) d \mathscr{L}^{n}(y) .
\end{aligned}
$$

We prove (7.4) by induction on $M$. If $M=1$, formula (7.4) becomes

$$
\begin{aligned}
\int_{\Omega_{\varepsilon}}\left(D_{-k} \Theta_{-k}^{(N)}\right)(y-x) \odot & u(y) d \mathscr{L}^{n}(y) \\
= & \int_{\partial \Omega_{\varepsilon}} \Theta_{-k}^{(N)}(y-x) \odot v(y) \odot u(y) d \mathscr{H}^{n-1}(y) \\
& \quad-\int_{\Omega_{\varepsilon}} \Theta_{-k}^{(N)}(y-x) \odot D_{k} u(y) d \mathscr{L}^{n}(y) .
\end{aligned}
$$

This follows immediately from (3.39) applied on the domain $\Omega_{\varepsilon}$, with $\Theta_{-k}^{(N)}(\cdot-x)$ in place of $u$, and with $u$ in place of $v$. Observe that this also proves (7.4) for $N=1$.

Next, assume that $N \geq 2$. Suppose (7.4) holds for some $M \in\{1,2, \ldots, N-1\}$ and the goal is to prove that (7.4) holds with $M$ replaced by $M+1$. In order to apply 
(3.39) and the induction hypothesis we write

$$
\begin{aligned}
& \int_{\Omega_{\varepsilon}}\left(D_{-k}^{M+1} \Theta_{-k}^{(N)}\right)(y-x) \odot u(y) d \mathscr{L}^{n}(y) \\
& =\int_{\Omega_{\varepsilon}} D_{-k}\left[\left(D_{-k}^{M} \Theta_{-k}^{(N)}\right)(y-x)\right] \odot u(y) d \mathscr{L}^{n}(y) \\
& =\int_{\partial \Omega_{\varepsilon}}\left(D_{-k}^{M} \Theta_{-k}^{(N)}\right)(y-x) \odot v(y) \odot u(y) d \mathscr{H}^{n-1}(y) \\
& -\int_{\Omega_{\varepsilon}}\left(D_{-k}^{M} \Theta_{-k}^{(N)}\right)(y-x) \odot D_{k} u(y) d \mathscr{L}^{n}(y) \\
& =\int_{\partial \Omega_{\varepsilon}} \Theta_{-k}^{(N-M)}(y-x) \odot v(y) \odot u(y) d \mathscr{H}^{n-1}(y) \\
& -\sum_{j=0}^{M-1}(-1)^{j} \int_{\partial \Omega_{\varepsilon}} \Theta_{-k}^{(N-M+j+1)}(y-x) \odot v(y) \odot D_{k}^{j} D_{k} u(y) d \mathscr{H}^{n-1}(y) \\
& -(-1)^{M} \int_{\Omega_{\varepsilon}} \Theta_{-k}^{(N)}(y-x) \odot D_{k}^{M} D_{k} u(y) d \mathscr{L}^{n}(y) \\
& =\int_{\partial \Omega_{\varepsilon}} \Theta_{-k}^{(N-M)}(y-x) \odot v(y) \odot u(y) d \mathscr{H}^{n-1}(y) \\
& +\sum_{j=1}^{M}(-1)^{j} \int_{\partial \Omega_{\varepsilon}} \Theta_{-k}^{(N-M+j)}(y-x) \odot v(y) \odot D_{k}^{j} u(y) d \mathscr{H}^{n-1}(y) \\
& +(-1)^{M+1} \int_{\Omega_{\varepsilon}} \Theta_{-k}^{(N)}(y-x) \odot D_{k}^{M+1} u(y) d \mathscr{L}^{n}(y) \\
& =\sum_{j=0}^{M}(-1)^{j} \int_{\partial \Omega_{\varepsilon}} \Theta_{-k}^{(N-M+j)}(y-x) \odot v(y) \odot D_{k}^{j} u(y) d \mathscr{H}^{n-1}(y) \\
& +(-1)^{M+1} \int_{\Omega_{\varepsilon}} \Theta_{-k}^{(N)}(y-x) \odot D_{k}^{M+1} u(y) d \mathscr{L}^{n}(y) .
\end{aligned}
$$

For the second equality in (7.6) we applied (3.39) (keeping in mind (7.2)), the third uses (6.27) and the induction hypothesis, while the fourth one is obtained by changing $j+1$ into $j$ in the respective summation. This proves that (7.4) also holds 
with $M$ replaced by $M+1$, concluding the proof of (7.4) (hence that of (7.1)).

An important consequence of Lemma 7.1 corresponding to the case when $M=N$ is stated next.

Corollary 7.2. Fix $k \in(0, \infty), N \in \mathbb{N}$, and suppose $\Omega$ is an open bounded subset of $\mathbb{R}^{n}$ with Lipschitz boundary $\partial \Omega$ and outward unit normal $v$. Consider the $C \ell_{m}$-valued function

$$
u \in \mathscr{C}^{\mathrm{N}}\left(\bar{\Omega}, C \ell_{m}\right) \text { satisfying } D_{k}^{N} u=0 \text { in } \Omega \text {. }
$$

Then $u$ has the integral representation formula

$$
u(x)=-\int_{\partial \Omega} \sum_{j=0}^{N-1}\left\{\Theta_{k}^{(j+1)}(x-y) \odot v(y) \odot D_{k}^{j} u(y)\right\} d \mathscr{H}^{n-1}(y) \quad \text { for every } x \in \Omega .
$$

Proof. Fix $x \in \Omega$ and consider an arbitrary $\varepsilon \in\left(0, \frac{1}{2} \operatorname{dist}(x, \partial \Omega)\right)$. Set $\Omega_{\varepsilon}:=\Omega \backslash \overline{B(x, \varepsilon)}$. Making use of (6.32) and then applying (7.1) combined with (7.7) yields

$$
\begin{aligned}
0= & \int_{\Omega_{\varepsilon}}\left(D_{-k}^{N} \Theta_{-k}^{(N)}\right)(y-x) \odot u(y) d \mathscr{L}^{n}(y) \\
= & \sum_{j=0}^{N-1}(-1)^{j} \int_{\partial \Omega} \Theta_{-k}^{(j+1)}(y-x) \odot v(y) \odot D_{k}^{j} u(y) d \mathscr{H}^{n-1}(y) \\
& -\sum_{j=0}^{N-1}(-1)^{j} \int_{\partial B(x, \varepsilon)} \Theta_{-k}^{(j+1)}(y-x) \odot \frac{y-x}{\varepsilon} \odot D_{k}^{j} u(y) d \mathscr{H}^{n-1}(y) .
\end{aligned}
$$

Since $u \in \mathscr{C}^{\mathrm{N}}\left(\bar{\Omega}, C \ell_{m}\right)$ there exists some $C \in(0, \infty)$ independent of $j$ such that $\left|D_{k}^{j} u\right| \leq C$ on $\bar{\Omega}$ for all $j \in\{0,1, \ldots, N\}$. This, combined with the first line in (6.17) 
allows us to estimate

$$
\begin{aligned}
& \left|\int_{\partial B(x, \varepsilon)} \Theta_{-k}^{(j+1)}(y-x) \odot \frac{y-x}{\varepsilon} \odot D_{k}^{j} u(y) d \mathscr{H}^{n-1}(y)\right| \\
& \leq C \int_{\partial B(x, \varepsilon)}\left|\Theta_{-k}^{(j+1)}(y-x)\right| d \mathscr{H}^{n-1}(y)=o(1) \text { as } \varepsilon \rightarrow 0^{+},
\end{aligned}
$$

for every $j \in\{1, \ldots, N-1\}$. Next, we analyze the integral in (7.9) over $\partial B(x, \varepsilon)$ corresponding to $j=0$ and write

$$
\begin{aligned}
\int_{\partial B(x, \varepsilon)} & \Theta_{-k}^{(1)}(y-x) \odot \frac{y-x}{\varepsilon} \odot u(y) d \mathscr{H}^{n-1}(y) \\
= & \int_{\partial B(x, \varepsilon)} \Theta_{-k}^{(1)}(y-x) \odot \frac{y-x}{\varepsilon} \odot(u(y)-u(x)) d \mathscr{H}^{n-1}(y) \\
& +\left(\int_{\partial B(x, \varepsilon)} \Theta_{-k}^{(1)}(y-x) \odot \frac{y-x}{\varepsilon} d \mathscr{H}^{n-1}(y)\right) \odot u(x) \\
= & : I_{1}(\varepsilon)+I_{2}(\varepsilon) .
\end{aligned}
$$

By the Mean Value Theorem, $u(y)-u(x)=O(\varepsilon)$ for $y \in \partial B(x, \varepsilon)$, which in concert with the second line in (6.17) gives

$$
I_{1}(\varepsilon)=o(1) \text { as } \varepsilon \rightarrow 0^{+}
$$

To analyze $I_{2}(\varepsilon)$, first observe that

$$
\begin{aligned}
\int_{\partial B(x, \varepsilon)}(y-x) d \mathscr{H}^{n-1}(y) & =\varepsilon^{n-1} \int_{\partial B(0,1)} z d \mathscr{H}^{n-1}(z) \\
& =\varepsilon^{n-1} \sum_{\ell=1}^{n}\left(\int_{\partial B(0,1)} z_{\ell} d \mathscr{H}^{n-1}(z)\right) e_{\ell}=0,
\end{aligned}
$$

where the last equality follows from [12, Proposition 13.49, p. 442]. Using (6.5), 
(4.2), (7.13), and the identity $\omega_{n-1}=\frac{2 \pi^{n / 2}}{\Gamma(n / 2)}($ see $[12,(13.5 .6), p .434])$ yields

$$
\begin{aligned}
& I_{2}(\varepsilon)=\left(\int_{\partial B(x, \varepsilon)} \frac{k^{n / 2}}{4 i(2 \pi)^{(n-2) / 2} \varepsilon^{(n-2) / 2}}\left[H_{n / 2}^{(1)}(k \varepsilon) \widehat{y-x}+H_{(n-2) / 2}^{(1)}(k \varepsilon) e_{n+1}\right]\right. \\
&\left.\odot \frac{y-x}{\varepsilon} d \mathscr{H}^{n-1}(y)\right) \odot u(x) \\
&=\frac{-k^{n / 2} H_{n / 2}^{(1)}(k \varepsilon)}{2^{(n+2) / 2} i \pi^{(n-2) / 2} \varepsilon^{(n-2) / 2}}\left(\int_{\partial B(x, \varepsilon)} d \mathscr{H}^{n-1}(y)\right) \odot u(x) \\
& \quad+\frac{k^{n / 2} H_{(n-2) / 2}^{(1)}(k \varepsilon)}{4 i(2 \pi)^{(n-2) / 2} \varepsilon^{n / 2}} e_{n+1} \odot\left(\int_{\partial B(x, \varepsilon)}(y-x) d \mathscr{H}^{n-1}(y)\right) \odot u(x) \\
&=\frac{-k^{n / 2} H_{n / 2}^{(1)}(k \varepsilon)}{2^{(n+2) / 2} i \pi^{(n-2) / 2} \varepsilon^{(n-2) / 2}} \varepsilon^{n-1} \omega_{n-1} u(x) \\
&=\frac{-(k \varepsilon)^{n / 2} H_{n / 2}^{(1)}(k \varepsilon)}{2^{n / 2} i \pi^{-1} \Gamma(n / 2)} u(x) \\
&=\frac{H_{n / 2}^{(1)}(k \varepsilon)}{\frac{2^{n / 2}}{i \pi} \Gamma(n / 2)(k \varepsilon)^{-n / 2}} u(x) \rightarrow u(x) \quad \text { as } \varepsilon \rightarrow 0^{+},
\end{aligned}
$$

where the convergence in (7.14) is a consequence of the second limit in (A.12). Returning to (7.9), we take the limit as $\varepsilon \rightarrow 0^{+}$use (7.10), (7.11), (7.12), and (7.14), to conclude that

$$
\sum_{j=0}^{N-1}(-1)^{j} \int_{\partial \Omega} \Theta_{-k}^{(j+1)}(y-x) \odot v(y) \odot D_{k}^{j} u(y) d \mathscr{H}^{n-1}(y)=u(x) .
$$

Now (7.8) follows from (7.15) by invoking (6.7).

Moving forward, to streamline the discussion we introduce operators that will play an important role in defining radiation conditions for null-solutions to iterated Dirac operators. We use the standard convention that a summation is zero if its upper bound of summation is less than its lower bound of summation. 
Definition 7.3. Let $N \in \mathbb{N}$ and $y \in \mathbb{R}^{n}$ be arbitrary. Define the $(N-1)^{\text {th }}$-order $C l_{m^{-}}$ differential operator $P_{N}\left(y ; D_{k}\right)$ as follows:

$$
\begin{aligned}
P_{N}\left(y ; D_{k}\right):= & \sum_{j=0}^{(N-2) / 2}\left[\frac{(-1)^{j}|y|^{j}}{j !(2 i k)^{j}}\left(i k+k e_{n+1} \odot \widehat{y}\right) \odot D_{k}^{2 j}\right] \\
& +\sum_{j=0}^{(N-2) / 2}\left[\frac{(-1)^{j}|y|^{j}}{j !(2 i k)^{j}} \widehat{y} \odot D_{k}^{2 j+1}\right] \quad \text { if } N \text { is even, }
\end{aligned}
$$

and

$$
\begin{aligned}
P_{N}\left(y ; D_{k}\right):= & \sum_{j=0}^{(N-1) / 2}\left[\frac{(-1)^{j}|y|^{j}}{j !(2 i k)^{j}}\left(i k+k e_{n+1} \odot \widehat{y}\right) \odot D_{k}^{2 j}\right] \\
& +\sum_{j=0}^{(N-3) / 2}\left[\frac{(-1)^{j}|y|^{j}}{j !(2 i k)^{j}} \widehat{y} \odot D_{k}^{2 j+1}\right] \quad \text { if } N \text { is odd. }
\end{aligned}
$$

We make a few remarks regarding the operators in (7.16)-(7.17). First, since $e_{n+1} \odot \widehat{y}=-\widehat{y} \odot e_{n+1}$, we have

$$
i k+k e_{n+1} \odot \widehat{y}+\widehat{y} \odot D_{k}=i k+\widehat{y} \odot D .
$$

Hence, we may rewrite the expressions for $P_{N}\left(y ; D_{k}\right)$ in the form

$$
P_{N}\left(y ; D_{k}\right)=\left\{\begin{array}{cc}
\sum_{j=0}^{(N-2) / 2}\left[\frac{(-1)^{j}|y|^{j}}{j !(2 i k)^{j}}(i k+\widehat{y} \odot D) \odot D_{k}^{2 j}\right] & \text { if } N \text { is even, } \\
\sum_{j=0}^{(N-3) / 2}\left[\frac{(-1)^{j}|y|^{j}}{j !(2 i k)^{j}}(i k+\widehat{y} \odot D) \odot D_{k}^{2 j}\right] & \\
+\frac{(-1)^{\frac{N-1}{2}}|y|^{\frac{N-1}{2}}}{\left(\frac{N-1}{2}\right) !(2 i k)^{\frac{N-1}{2}}}\left(i k+k e_{n+1} \odot \widehat{y}\right) \odot D_{k}^{N-1} & \text { if } N \text { is odd. }
\end{array}\right.
$$


Second, if we also make use of (2.27), the expressions for $P_{N}\left(y ; D_{k}\right)$ take the form

$$
P_{N}\left(y ; D_{k}\right)= \begin{cases}\sum_{j=0}^{(N-2) / 2}\left[\frac{|y|^{j}}{j !(2 i k)^{j}}(i k+\widehat{y} \odot D) \odot\left(\Delta+k^{2}\right)^{j}\right] & \text { if } N \text { is even, } \\ \sum_{j=0}^{(N-3) / 2}\left[\frac{|y|^{j}}{j !(2 i k)^{j}}(i k+\widehat{y} \odot D) \odot\left(\Delta+k^{2}\right)^{j}\right] & \\ +\frac{|y|^{\frac{N-1}{2}}}{\left(\frac{N-1}{2}\right) !(2 i k)^{\frac{N-1}{2}}}\left(i k+k e_{n+1} \odot \widehat{y}\right) \odot\left(\Delta+k^{2}\right)^{\frac{N-1}{2}} & \text { if } N \text { is odd. }\end{cases}
$$

Definition 7.4. Let $N \in \mathbb{N}_{0}$ and $y \in \mathbb{R}^{n}$ be arbitrary. Define the $(N-1)^{\text {th }}$-order $C \ell_{m^{-}}$ differential operator $Q_{N}\left(y ; D_{k}\right)$ by

$$
Q_{N}\left(y ; D_{k}\right):=\left\{\begin{array}{cc}
(i k+\widehat{y} \odot D) \odot \sum_{j=0}^{(N-2) / 2}\left[\frac{(-1)^{j}|y|^{j}}{j !(2 i k)^{j}} D_{k}^{2 j}\right] & \text { if } N \text { is even, } \\
(i k+\widehat{y} \odot D) \odot \sum_{j=0}^{(N-3) / 2}\left[\frac{(-1)^{j}|y|^{j}}{j !(2 i k)^{j}} D_{k}^{2 j}\right] & \\
+\frac{(-1)^{\frac{N-1}{2}}|y|^{\frac{N-1}{2}}}{\left(\frac{N-1}{2}\right) !(2 i k)^{\frac{N-1}{2}}}\left(i k+k e_{n+1} \odot \widehat{y}\right) \odot D_{k}^{N-1} & \text { if } N \text { is odd. }
\end{array}\right.
$$

Remark 7.5. In light of (2.27), we also have

$$
Q_{N}\left(y ; D_{k}\right):= \begin{cases}(i k+\widehat{y} \odot D) \odot \sum_{j=0}^{(N-2) / 2}\left[\frac{|y|^{j}}{j !(2 i k)^{j}}\left(\Delta+k^{2}\right)^{j}\right] & \text { if } N \text { is even, } \\ (i k+\widehat{y} \odot D) \odot \sum_{j=0}^{(N-3) / 2}\left[\frac{|y|^{j}}{j !(2 i k)^{j}}\left(\Delta+k^{2}\right)^{j}\right] & \\ +\frac{|y|^{\frac{N-1}{2}}}{\left(\frac{N-1}{2}\right) !(2 i k)^{\frac{N-1}{2}}}\left(i k+k e_{n+1} \odot \widehat{y}\right) \odot\left(\Delta+k^{2}\right)^{\frac{N-1}{2}} & \text { if } N \text { is odd. }\end{cases}
$$


Definition 7.6. Fix $k \in(0, \infty), N \in \mathbb{N}$, and let $\Omega$ be an exterior domain in $\mathbb{R}^{n}$. Recall the $C l_{m}$-differential operator $P_{N}\left(y ; D_{k}\right)$ in (7.16). Consider a $C \ell_{m}$-valued function $u \in$ $\mathscr{C}^{N-1}\left(\Omega, C \ell_{m}\right)$. We say that $u$ is radiating in $\Omega$ provided

$$
\int_{|y|=R}\left|P_{N}\left(y ; D_{k}\right) u(y)\right|^{2} d \mathscr{H}^{n-1}(y)=o(1) \quad \text { as } \quad R \rightarrow \infty
$$

and

$$
\int_{|y|=R}\left|D_{k}^{j} u(y)\right|^{2} d \mathscr{H}^{n-1}(y)=\left\{\begin{array}{ll}
o\left(R^{2-j}\right) & \text { if } j \text { is even, } \\
o\left(R^{3-j}\right) & \text { if } j \text { is odd }
\end{array} \text { as } R \rightarrow \infty,\right.
$$

for all $j \in\{0,1, \ldots, N-1\}$.

Remark 7.7. Fix $k \in(0, \infty), N \in \mathbb{N}$ and an exterior domain $\Omega$ in $\mathbb{R}^{n}$. Suppose $u$ is a $C \ell_{m}$-valued function satisfying

$$
u \in \mathscr{C}^{N-1}\left(\Omega, C \ell_{m}\right) \text { and } D_{k}^{N} u=0 \text { in } \Omega \text {. }
$$

(1) When $N=1$, condition (7.23) becomes

$$
\int_{|y|=R}\left|\left(i+e_{n+1} \odot \widehat{y}\right) \odot u(y)\right|^{2} d \mathscr{H}^{n-1}(y)=o(1) \quad \text { as } \quad R \rightarrow \infty .
$$

By [9, Corollary 1.5, part (A)], for every $u$ as in (7.25) we have that $u$ satisfies (7.26) if and only if $u$ satisfies

$$
\left(i+e_{n+1} \odot \widehat{y}\right) \odot u(y)=o\left(|x|^{-(n-1) / 2}\right) \quad \text { as } \quad|y| \rightarrow \infty .
$$

The latter is the radiation condition identified in [11]. 
(2) When $N=2$, condition (7.23) becomes (keeping in mind (7.20))

$$
\int_{|y|=R}|i k u(y)+\widehat{y} \odot D u(y)|^{2} d \mathscr{H}^{n-1}(y)=o(1) \quad \text { as } \quad R \rightarrow \infty .
$$

According to [9, Theorem 1.1, part (A)], if $u$ is as in (7.25) then $u$ satisfies condition (7.28) if and only if

$$
i k u(y)-\sum_{j=1}^{n} \widehat{y}_{j}\left(\partial_{j} u\right)(y)=o\left(|y|^{-(n-1) / 2}\right) \text { as }|y| \rightarrow \infty
$$

The latter is precisely the Sommerfeld radiation condition (1.1) for the Helmholtz operator. Moreover, from [9, Theorem 1.1, part (A)], we know that for every $u$ as in (7.25), condition (7.28) holds if and only if

$$
\int_{|y|=R}\left|i k u(y)-\sum_{j=1}^{n} \widehat{y}_{j}\left(\partial_{j} u\right)(y)\right|^{2} d \mathscr{H}^{n-1}(y)=o(1) \quad \text { as } \quad R \rightarrow \infty
$$

(3) Regarding (7.24), first observe that if $N=1$ and $u$ is as in (7.25), then

$$
\begin{aligned}
P_{1}\left(y ; D_{k}\right) u(y) & =\left(i k+k e_{n+1} \odot \widehat{y}\right) \odot u(y) \\
& =i k u(y)-\widehat{y} \odot k e_{n+1} \odot u(y) \\
& =i k u(y)+\widehat{y} \odot D u(y)
\end{aligned}
$$

for every $y \in \Omega$. Invoking now [9, Lemma 5.1] (which is stated below as Lemma 7.8), we see that if $u$ also satisfies (7.23) then condition (7.24) holds.

Corresponding to the case $N=2$, since $P_{2}\left(y ; D_{k}\right)=i k+\widehat{y} \odot D$, again by [9, Lemma 5.1] we have that any $u$ as in (7.25) that satisfies (7.23) will also satisfy (7.24). What happens when $N \geq 3$ is still an open question. 
(4) A direct computation gives that, for each $y \in \Omega$,

$$
\widehat{y} \odot D u(y)=-\sum_{j=1}^{n} \widehat{y}_{j}\left(\partial_{j} u\right)(y)+\sum_{j, k=1, j<k}^{n} e_{j} \odot e_{k} \odot\left(\widehat{y}_{j}\left(\partial_{k} u\right)(y)-\widehat{y}_{k}\left(\partial_{j} u\right)(y)\right) .
$$

Given this, it is remarkable that, as proved in [9, Corrolary $1.3(v)]$, if $u$ is as in (7.25) and satisfies (7.28) then necessarily $u$ satisfies the following conditions

$$
\begin{gathered}
\int_{|y|=R}\left|\widehat{y}_{j}\left(\partial_{k} u\right)(y)-\widehat{y}_{k}\left(\partial_{j} u\right)(y)\right|^{2} d \mathscr{H}^{n-1}(y)=o(1) \\
\text { as } \quad R \rightarrow \infty . \quad \forall j, k \in\{1, \ldots, n\} .
\end{gathered}
$$

(5) While both (7.23) and (7.24) express growth/decay conditions for powers of the perturbed Dirac operator acting on some $u \in \mathscr{C}^{N-1}\left(\Omega, C \ell_{m}\right)$, condition (7.23) is not implied by (7.24) as already visible from the inspection of the case $N=2$ (as discussed above). In general, estimating $\int_{|y|=R}\left|P_{N}\left(y ; D_{k}\right) u(y)\right|^{2} d \mathscr{H}^{n-1}(y)$ via the conditions in (7.24) leads to a less stringent decay condition than (7.23). So, from this perspective, (7.23) is the most delicate condition. Its veracity is affected even by the manner in which the terms in $P_{N}\left(y ; D_{k}\right) u$ combine algebraically with one another, i.e., internal cancellations are important (much as in the classical Sommerfeld radiation condition).

Here is the lemma alluded to in item (3) of Remark 7.7.

Lemma 7.8. [9] Fix $k \in(0, \infty), m \in \mathbb{N}_{0}$, and $n \in \mathbb{N}$ with $n \geq 2$. Let $\Omega$ be an exterior domain in $\mathbb{R}^{n}$. Suppose $u \in \mathscr{C}^{\infty}\left(\Omega, C \ell_{m}\right)$ satisfies $\left(\Delta+k^{2}\right) u=0$ in $\Omega$ and

$$
\int_{|y|=R}|i k u(y)+\widehat{y} \odot(D u)(y)|^{2} d \mathscr{H}^{n-1}(y)=O(1) \text { as } R \rightarrow \infty \text {. }
$$

Then

$$
\int_{|y|=R}|u(y)|^{2} d \mathscr{H}^{n-1}(y)=O(1) \text { as } R \rightarrow \infty
$$


and

$$
\int_{|y|=R}|D u(y)|^{2} d \mathscr{H}^{n-1}(y)=O(1) \text { as } R \rightarrow \infty .
$$

In the next theorem we prove that in the definition of a radiating function, condition (7.23) may be replaced by another decay condition involving the operators $Q_{N}\left(y ; D_{k}\right)$

Theorem 7.9. Fix $k \in(0, \infty), N \in \mathbb{N}$, and let $\Omega$ be an exterior domain in $\mathbb{R}^{n}$. Recall the $C \ell_{m}$-differential operator $Q_{N}\left(y ; D_{k}\right)$ in (7.21). Consider a $C \ell_{m}$-valued function $u \in$ $\mathscr{C}^{N-1}\left(\Omega, C \ell_{m}\right)$. Then $u$ is radiating in $\Omega$ if and only if $u$ satisfies (7.24) and

$$
\int_{|y|=R}\left|Q_{N}\left(y ; D_{k}\right) u(y)\right|^{2} d \mathscr{H}^{n-1}(y)=o(1) \quad \text { as } \quad R \rightarrow \infty .
$$

Proof. Let us assume for the moment that $u \in \mathscr{C}^{\infty}\left(\Omega, C \ell_{m}\right)$. Then a direct computation applying the product rule and (5.7) yields

$$
|y|^{j} D\left[D_{k}^{2 j} u(y)\right]=D\left[|y|^{j} D_{k}^{2 j} u(y)\right]-j|y|^{j-1} \widehat{y} \odot D_{k}^{2 j} u(y)
$$

for each $j \in \mathbb{N}$ and each $y \in \Omega$. Consequently, (recall also (2.1))

$$
|y|^{j}(i k+\widehat{y} \odot D)\left[D_{k}^{2 j} u(y)\right]-(i k+\widehat{y} \odot D)\left[|y|^{j} D_{k}^{2 j} u(y)\right]=j|y|^{j-1} D_{k}^{2 j} u(y)
$$

for each $j \in \mathbb{N}$ and each $y \in \Omega$. With the help of (7.39), for each $M \in \mathbb{N}_{0}$ we may 
now write

$$
\begin{gathered}
\sum_{j=0}^{M} \frac{(-1)^{j}|y|^{j}}{j !(2 i k)^{j}}(i k+\widehat{y} \odot D) D_{k}^{2 j} u(y)-(i k+\widehat{y} \odot D) \sum_{j=0}^{M} \frac{(-1)^{j}|y|^{j}}{j !(2 i k)^{j}} D_{k}^{2 j} u(y) \\
=\sum_{j=1}^{M} \frac{(-1)^{j}|y|^{j-1}}{(j-1) !(2 i k)^{j}} D_{k}^{2 j} u(y), \quad \forall y \in \Omega .
\end{gathered}
$$

Returning to the assumption $u \in \mathscr{C}^{N-1}\left(\Omega, C \ell_{m}\right)$, set $M:=(N-2) / 2$ if $N$ is even and $M:=(N-3) / 2$ if $N$ is odd. Based on (7.19), (7.21), and (7.40) we may write

$$
P_{N}\left(y ; D_{k}\right)-Q_{N}\left(y ; D_{k}\right)=\sum_{j=1}^{M} \frac{(-1)^{j}|y|^{j-1}}{(j-1) !(2 i k)^{j}} D_{k}^{2 j} u(y), \quad \forall y \in \Omega .
$$

Consequently, there exists some constant $C=C(N, n, k)$ such that, for each $R>0$ sufficiently large,

$$
\begin{aligned}
\int_{|y|=R} \mid P_{N}\left(y ; D_{k}\right) & -\left.Q_{N}\left(y ; D_{k}\right)\right|^{2} d \mathscr{H}^{n-1}(y) \\
& \leq C \sum_{j=1}^{M} \int_{|y|=R}|y|^{2 j-2}\left|D_{k}^{2 j} u(y)\right|^{2} d \mathscr{H}^{n-1}(y) \\
& =C \sum_{j=1}^{M} R^{2 j-2} \int_{|y|=R}\left|D_{k}^{2 j} u(y)\right|^{2} d \mathscr{H}^{n-1}(y) .
\end{aligned}
$$

Bringing in the assumption that $u$ satisfies (7.24), from (7.42) we may conclude that

$$
\int_{|y|=R}\left|P_{N}\left(y ; D_{k}\right)-Q_{N}\left(y ; D_{k}\right)\right|^{2} d \mathscr{H}^{n-1}(y)=o(1) \quad \text { as } \quad R \rightarrow \infty .
$$

Hence, if $u \in \mathscr{C}^{N-1}\left(\Omega, C \ell_{m}\right)$ is such that (7.24) holds, then $u$ satisfies (7.23) if and only if $u$ satisfies (7.37). This proves the theorem. 
Lemma 7.10. Fix $k \in(0, \infty), N \in \mathbb{N}$, and let $\Omega$ be an exterior domain in $\mathbb{R}^{n}$. Suppose $u \in \mathscr{C}^{N-1}\left(\Omega, C \ell_{m}\right)$ is radiating in $\Omega$. Then, for any $x \in \Omega$,

$$
\int_{|y|=R} \sum_{j=0}^{N-1}\left\{\Theta_{k}^{(j+1)}(x-y) \odot \widehat{y} \odot D_{k}^{j} u(y)\right\} d \mathscr{H}^{n-1}(y)=o(1) \quad \text { as } \quad R \rightarrow \infty .
$$

Proof. Fix $x \in \Omega$. For each $j \in \mathbb{N}_{0}$ we introduce

$$
A_{j}(y):=\Theta_{k}^{(j+1)}(x-y) \odot \widehat{y} \odot D_{k}^{j} u(y)
$$

Recalling the constants $b_{n, k}^{(j+1)}=\frac{b_{n, k}}{j !(2 i k)^{j}}$ from (5.46), Lemma 6.6 tells us

$$
\begin{aligned}
A_{2 j}(y)= & {\left[(-1)^{(j+1)} k b_{n, k}^{(j+1)} \frac{e^{i k|y|} e^{-i k\langle x, \widehat{y}\rangle}}{|y|^{(n-2 j-1) / 2}}\left(-i \widehat{y}+e_{n+1}\right)\right.} \\
& \left.+O\left(|y|^{-(n-2 j+1) / 2}\right)\right] \odot \widehat{y} \odot D_{k}^{2 j} u(y) \\
= & \frac{(-1)^{(j+1)} b_{n, k} e^{i k|y|} e^{-i k\langle x, \widehat{y}\rangle}}{j !(2 i k)^{j}|y|^{(n-2 j-1) / 2}}\left(i k+k e_{n+1} \odot \widehat{y}\right) \odot D_{k}^{2 j} u(y) \\
& +O\left(|y|^{-(n-2 j+1) / 2}\right) \odot D_{k}^{2 j} u(y),
\end{aligned}
$$

and

$$
\begin{aligned}
A_{2 j+1}(y)= & {\left[(-1)^{(j+1)} b_{n, k}^{(j+1)} \frac{e^{i k|y|} e^{-i k\langle x, \widehat{y}\rangle}}{|y|^{(n-2 j-1) / 2}}\right.} \\
& \left.+O\left(|y|^{-(n-2 j+1) / 2}\right)\right] \odot \widehat{y} \odot D_{k}^{2 j+1} u(y) \\
= & \frac{(-1)^{(j+1)} b_{n, k} e^{i k|y|} e^{-i k\langle x, \widehat{y}\rangle}}{j !(2 i k)^{j}|y|^{(n-2 j-1) / 2}} \widehat{y} D_{k}^{2 j+1} u(y) \\
& +O\left(|y|^{-(n-2 j+1) / 2}\right) \odot D_{k}^{2 j+1} u(y)
\end{aligned}
$$


as $|y| \rightarrow \infty$. Consequently,

$$
\begin{aligned}
A_{2 j}(y) & +A_{2 j+1}(y) \\
& =\frac{(-1)^{(j+1)} b_{n, k} e^{i k|y|} e^{-i k\langle x, \widehat{y}\rangle}}{j !(2 i k)^{j}|y|^{(n-2 j-1) / 2}}\left[\left(i k+k e_{n+1} \odot \widehat{y}\right) \odot D_{k}^{2 j} u(y)+\widehat{y} \odot D_{k}^{2 j+1} u(y)\right] \\
& +O\left(|y|^{-(n-2 j+1) / 2}\right) \odot D_{k}^{2 j} u(y)+O\left(|y|^{-(n-2 j+1) / 2}\right) \odot D_{k}^{2 j+1} u(y),
\end{aligned}
$$

as $|y| \rightarrow \infty$.

Case $N$ is even: Suppose $N=2 M$ for some $M \in \mathbb{N}$. From (7.48) and (7.16) we see that

$$
\begin{aligned}
& \sum_{j=0}^{N-1}\left\{\Theta_{k}^{(j+1)}(x-y) \odot \widehat{y} \odot D_{k}^{j} u(y)\right\}=\sum_{j=0}^{2 M-1} A_{j}(y) \\
& =\sum_{j=0}^{M-1}\left(A_{2 j}(y)+A_{2 j+1}(y)\right) \\
& =\frac{b_{n, k} e^{i k|y|} e^{-i k\langle x, \widehat{y}\rangle}}{|y|^{(n-1) / 2}} \sum_{j=0}^{M-1} \frac{(-1)^{(j+1)}|y|^{j}}{j !(2 i k)^{j}}\left(i k+k e_{n+1} \odot \widehat{y}\right) \odot D_{k}^{2 j} u(y) \\
& +\frac{b_{n, k} e^{i k|y|} e^{-i k\langle x, \widehat{y}\rangle}}{|y|^{(n-1) / 2}} \sum_{j=0}^{M-1} \frac{(-1)^{(j+1)}|y|^{j}}{j !(2 i k)^{j}} \widehat{y} \odot D_{k}^{2 j+1} u(y) \\
& +\sum_{j=0}^{M-1} O\left(|y|^{-(n-2 j+1) / 2}\right) \odot D_{k}^{2 j} u(y)+\sum_{j=0}^{M-1} O\left(|y|^{-(n-2 j+1) / 2}\right) \odot D_{k}^{2 j+1} u(y) \\
& =\frac{-b_{n, k} e^{i k|y|} e^{-i k\langle x, \widehat{y}\rangle}}{|y|^{(n-1) / 2} P_{N}\left(y ; D_{k}\right) u(y)+\sum_{j=0}^{(N-2) / 2} O\left(|y|^{-(n-2 j+1) / 2}\right) \odot D_{k}^{2 j} u(y)} \\
& +\sum_{j=0}^{(N-2) / 2} O\left(|y|^{-(n-2 j+1) / 2}\right) \odot D_{k}^{2 j+1} u(y)
\end{aligned}
$$


as $|y| \rightarrow \infty$. Based on (7.49), for $R$ sufficiently large we may therefore estimate

$$
\begin{aligned}
\mid \int_{|y|=R} \sum_{j=0}^{N-1}\{ & \left.\Theta_{k}^{(j+1)}(x-y) \odot \widehat{y} \odot D_{k}^{j} u(y)\right\} d \mathscr{H}^{n-1}(y) \mid \\
\leq & \int_{|y|=R}\left|O\left(|y|^{-(n-1) / 2}\right) P_{N}\left(y ; D_{k}\right) u(y)\right| d \mathscr{H}^{n-1}(y) \\
& +\sum_{j=0}^{(N-2) / 2} \int_{|y|=R}\left|O\left(|y|^{-(n-2 j+1) / 2}\right) D_{k}^{2 j} u(y)\right| d \mathscr{H}^{n-1}(y) \\
& +\sum_{j=0}^{(N-2) / 2} \int_{|y|=R}\left|O\left(|y|^{-(n-2 j+1) / 2}\right) D_{k}^{2 j+1} u(y)\right| d \mathscr{H}^{n-1}(y) .
\end{aligned}
$$

Using the Cauchy-Schwarz inequality and (7.23) (since $u$ is radiating in $\Omega$ ) we see that

$$
\begin{aligned}
& \int_{|y|=R}\left|O\left(|y|^{-(n-1) / 2}\right) P_{N}\left(y ; D_{k}\right) u(y)\right| d \mathscr{H}^{n-1}(y) \\
& \quad \leq O(1)\left(\int_{|y|=R}\left|P_{N}\left(y ; D_{k}\right) u(y)\right|^{2} d \mathscr{H}^{n-1}(y)\right)^{1 / 2}=o(1) \quad \text { as } \quad R \rightarrow \infty .
\end{aligned}
$$

Moreover, the Cauchy-Schwarz inequality and the assumption corresponding to the even case in (7.24), imply

$$
\begin{aligned}
\int_{|y|=R} \mid & O\left(|y|^{-(n-2 j+1) / 2}\right) D_{k}^{2 j} u(y) \mid d \mathscr{H}^{n-1}(y) \\
& \leq\left(O\left(R^{-(n-2 j+1)+(n-1)}\right)\right)^{1 / 2}\left(\int_{|y|=R}\left|D_{k}^{2 j} u(y)\right|^{2} d \mathscr{H}^{n-1}(y)\right)^{1 / 2} \\
& =O\left(R^{j-1}\right) o\left(R^{1-j}\right)=o(1) \quad \text { as } \quad R \rightarrow \infty
\end{aligned}
$$

for each $j \in\{0,1, \ldots,(N-2) / 2\}$. Similarly, by the Cauchy-Schwarz inequality and 
the assumption corresponding to the odd case in (7.24), we have

$$
\int_{|y|=R}\left|O\left(|y|^{-(n-2 j+1) / 2}\right) D_{k}^{2 j+1} u(y)\right| d \mathscr{H}^{n-1}(y)=o(1) \quad \text { as } \quad R \rightarrow \infty
$$

for each $j \in\{0,1, \ldots,(N-2) / 2\}$. At this stage (7.44) follows by combining (7.50)(7.53).

Case $N$ is odd: Suppose $N=2 M+1$ for some $M \in \mathbb{N}_{0}$. From (7.46), (7.48), and 
(7.16) we find that

$$
\begin{aligned}
& \sum_{j=0}^{N-1}\left\{\Theta_{k}^{(j+1)}(x-y) \odot \widehat{y} \odot D_{k}^{j} u(y)\right\}=\sum_{j=0}^{2 M} A_{j}(x, y) \\
& =A_{2 M}(y)+\sum_{j=0}^{M-1}\left(A_{2 j}(x, y)+A_{2 j+1}(x, y)\right) \\
& =\frac{(-1)^{(M+1)} b_{n, k} e^{i k|y|} e^{-i k\langle x, \widehat{y}\rangle}}{M !(2 i k)^{M}|y|^{(n-2 M-1) / 2}}\left(i k+k e_{n+1} \odot \widehat{y}\right) \odot D_{k}^{2 M} u(y) \\
& +O\left(|y|^{-(n-2 M+1) / 2}\right) \odot D_{k}^{2 M} u(y) \\
& +\frac{b_{n, k} e^{i k|y|} e^{-i k\langle x, \widehat{y}\rangle}}{|y|^{(n-1) / 2}} \sum_{j=0}^{M-1} \frac{(-1)^{(j+1)}|y|^{j}}{j !(2 i k)^{j}}\left(i k+k e_{n+1} \odot \widehat{y}\right) \odot D_{k}^{2 j} u(y) \\
& +\frac{b_{n, k} e^{i k|y|} e^{-i k\langle x, \widehat{y}\rangle}}{|y|^{(n-1) / 2}} \sum_{j=0}^{M-1} \frac{(-1)^{(j+1)}|y|^{j}}{j !(2 i k)^{j}} \widehat{y} \odot D_{k}^{2 j+1} u(y) \\
& +\sum_{j=0}^{M-1} O\left(|y|^{-(n-2 j+1) / 2}\right) \odot D_{k}^{2 j} u(y)+\sum_{j=0}^{M-1} O\left(|y|^{-(n-2 j+1) / 2}\right) \odot D_{k}^{2 j+1} u(y) \\
& =\frac{b_{n, k} e^{i k|y|} e^{-i k\langle x, \widehat{y}\rangle}}{|y|^{(n-1) / 2}} \sum_{j=0}^{M} \frac{(-1)^{(j+1)}|y|^{j}}{j !(2 i k)^{j}}\left(i k+k e_{n+1} \odot \widehat{y}\right) \odot D_{k}^{2 j} u(y) \\
& +\frac{b_{n, k} e^{i k|y|} e^{-i k\langle x, \widehat{y}\rangle}}{|y|^{(n-1) / 2}} \sum_{j=0}^{M-1} \frac{(-1)^{(j+1)}|y|^{j}}{j !(2 i k)^{j}} \odot D_{k}^{2 j+1} u(y) \\
& +\sum_{j=0}^{M} O\left(|y|^{-(n-2 j+1) / 2}\right) \odot D_{k}^{2 j} u(y)+\sum_{j=0}^{M-1} O\left(|y|^{-(n-2 j+1) / 2}\right) \odot D_{k}^{2 j+1} u(y) \\
& =\frac{-b_{n, k} e^{i k|y|} e^{-i k\langle x, \widehat{y}\rangle}}{|y|^{(n-1) / 2}} P_{N}\left(y ; D_{k}\right) u(y)+\sum_{j=0}^{(N-1) / 2} O\left(|y|^{-(n-2 j+1) / 2}\right) \odot D_{k}^{2 j} u(y) \\
& +\sum_{j=0}^{(N-3) / 2} O\left(|y|^{-(n-2 j+1) / 2}\right) \odot D_{k}^{2 j+1} u(y)
\end{aligned}
$$


as $|y| \rightarrow \infty$. Based on (7.54), for $R$ sufficiently large we may therefore estimate

$$
\begin{aligned}
\mid \int_{|y|=R} \sum_{j=0}^{N-1}\{ & \left.\Theta_{k}^{(j+1)}(x-y) \odot \widehat{y} \odot D_{k}^{j} u(y)\right\} d \mathscr{H}^{n-1}(y) \mid \\
\leq & \int_{|y|=R}\left|O\left(|y|^{-(n-1) / 2}\right) P_{N}\left(y ; D_{k}\right) u(y)\right| d \mathscr{H}^{n-1}(y) \\
& +\sum_{j=0}^{(N-1) / 2} \int_{|y|=R}\left|O\left(|y|^{-(n-2 j+1) / 2}\right) D_{k}^{2 j} u(y)\right| d \mathscr{H}^{n-1}(y) \\
& +\sum_{j=0}^{(N-3) / 2} \int_{|y|=R}\left|O\left(|y|^{-(n-2 j+1) / 2}\right) D_{k}^{2 j+1} u(y)\right| d \mathscr{H}^{n-1}(y) .
\end{aligned}
$$

Using the Cauchy-Schwarz inequality and (7.23) we see that

$$
\begin{aligned}
& \int_{|y|=R}\left|O\left(|y|^{-(n-1) / 2}\right) P_{N}\left(y ; D_{k}\right) u(y)\right| d \mathscr{H}^{n-1}(y) \\
& \quad \leq O(1)\left(\int_{|y|=R}\left|P_{N}\left(y ; D_{k}\right) u(y)\right|^{2} d \mathscr{H}^{n-1}(y)\right)^{1 / 2}=o(1) \quad \text { as } \quad R \rightarrow \infty .
\end{aligned}
$$

Moreover, the Cauchy-Schwarz inequality and the assumption corresponding to the even case in (7.24), imply

$$
\begin{array}{rl}
\int_{|y|=R} & O\left(|y|^{-(n-2 j+1) / 2}\right) D_{k}^{2 j} u(y) \mid d \mathscr{H}^{n-1}(y) \\
& \leq\left(O\left(R^{-(n-2 j+1)+(n-1)}\right)\right)^{1 / 2}\left(\int_{|y|=R}\left|D_{k}^{2 j} u(y)\right|^{2} d \mathscr{H}^{n-1}(y)\right)^{1 / 2} \\
& =O\left(R^{j-1}\right) o\left(R^{1-j}\right)=o(1) \quad \text { as } \quad R \rightarrow \infty
\end{array}
$$

for each $j \in\{0,1, \ldots,(N-1) / 2\}$. Similarly, by the Cauchy-Schwarz inequality and 
the assumption corresponding to the odd case in (7.24), we have

$$
\int_{|y|=R}\left|O\left(|y|^{-(n-2 j+1) / 2}\right) D_{k}^{2 j+1} u(y)\right| d \mathscr{H}^{n-1}(y)=o(1) \quad \text { as } \quad R \rightarrow \infty
$$

for each $j \in\{0,1, \ldots,(N-3) / 2\}$. At this stage (7.44) follows by combining (7.55)(7.58).

We are now ready to state and prove an integral representation formula for radiating null-solutions of the iterated perturbed Dirac operator in Lipschitz domains.

Theorem 7.11. Fix $k \in(0, \infty), N \in \mathbb{N}$, and let $\Omega$ be an exterior domain in $\mathbb{R}^{n}$ with Lipschitz boundary $\partial \Omega$ and outward unit normal $v$. Consider a $C \ell_{m}$-valued function

$$
u \in \mathscr{C}^{N}\left(\bar{\Omega}, C \ell_{m}\right) \text { satisfying } D_{k}^{N} u=0 \text { in } \Omega
$$

Assume $u$ is radiating in $\Omega$. Then $u$ has the integral representation formula

$$
u(x)=-\int_{\partial \Omega} \sum_{j=0}^{N-1}\left\{\Theta_{k}^{(j+1)}(x-y) \odot v(y) \odot D_{k}^{j} u(y)\right\} d \mathscr{H}^{n-1}(y) \quad \text { for every } x \in \Omega .
$$

Proof. Fix $x \in \Omega$, and consider an $R \in \mathbb{R}$ large enough so that $x \in B(0, R)$ and $\mathbb{R}^{n} \backslash \Omega \subseteq B(0, R)$. For simplicity of notation, set $\Omega_{R}:=\Omega \cap B(0, R)$. Thus, $\partial \Omega_{R}=$ $\partial \Omega \cup \partial B(0, R)$ and if we continue to use $v$ to denote the outward unit normal to $\Omega_{R}$, then $v(y)=\widehat{y}$ for every $y \in \partial B(0, R)$. Since $\Omega_{R} \subseteq B(0, R)$ and $\Omega_{R} \subseteq \Omega$ we may apply 
Corollary 7.2 resulting in

$$
\begin{aligned}
u(x) & =-\int_{\partial \Omega_{R}} \sum_{j=0}^{N-1}\left\{\Theta_{k}^{(j+1)}(x-y) \odot v(y) \odot D_{k}^{j} u(y)\right\} d \mathscr{H}^{n-1}(y) \\
& =-\int_{\partial \Omega} \sum_{j=0}^{N-1}\left\{\Theta_{k}^{(j+1)}(x-y) \odot v(y) \odot D_{k}^{j} u(y)\right\} d \mathscr{H}^{n-1}(y)-Z
\end{aligned}
$$

where

$$
Z:=\int_{|y|=R} \sum_{j=0}^{N-1}\left\{\Theta_{k}^{(j+1)}(x-y) \odot \widehat{y} \odot D_{k}^{j} u(y)\right\} d \mathscr{H}^{n-1}(y) .
$$

From (7.61) it is seen that $Z$ is independent of $R$, and Lemma 7.10 tells us that $Z=o(1)$ as $R \rightarrow \infty$, so we conclude $Z \equiv 0$, completing our proof.

Remark 7.12. In the case $N=1$, by recalling parts (1) and (3) in Remark 7.7, we see that Theorem 7.11 states that if $u \in \mathscr{C}^{1}\left(\bar{\Omega}, C \ell_{m}\right)$ is $k$-monogenic (i.e. $D_{k} u=0$ in $\Omega$ ) and satisfies the decay condition (7.27) then, for every $x \in \Omega$, $u$ has the integral representation formula

$$
\begin{aligned}
u(x) & =-\int_{\partial \Omega} \Theta_{k}^{(1)}(x-y) \odot v(y) \odot u(y) d \mathscr{H}^{n-1}(y) \\
& =\int_{\partial \Omega}\left(D_{k} \Phi_{k}\right)(x-y) \odot v(y) \odot u(y) d \mathscr{H}^{n-1}(y),
\end{aligned}
$$

where (6.1) is applied in the second equality. This matches the integral representation formula presented in [10, Theorem 2.3] for a k-monogenic function. 


\section{Radiation conditions and integral representations for null-solutions of iterated Helmholtz operators}

In this section we obtain radiation conditions and integral representations for null-solutions of iterated Helmholtz operators. The main ingredient is Theorem 7.11 used for $N$ even.

Theorem 8.1. Fix $k \in(0, \infty), N \in \mathbb{N}$, and let $\Omega$ be an exterior domain in $\mathbb{R}^{n}$ with Lipschitz boundary $\partial \Omega$ and outward unit normal $v$. Consider a $C \ell_{m}$-valued function

$$
u \in \mathscr{C}^{2 N}\left(\bar{\Omega}, C \ell_{m}\right) \text { satisfying }\left(\Delta+k^{2}\right)^{N} u=0 \text { in } \Omega \text {. }
$$

Suppose $u$ satisfies the radiation condition

$$
\int_{|y|=R}\left|i k w_{N}(y)+\widehat{y} \odot D w_{N}(y)\right|^{2} d \mathscr{H}^{n-1}(y)=o(1) \quad \text { as } \quad R \rightarrow \infty \text {, }
$$

where,

$$
w_{N}(y):=\sum_{j=0}^{N-1} \frac{|y|^{j}}{j !(2 i k)^{j}}\left(\Delta+k^{2}\right)^{j} u(y), \quad \forall y \in \Omega .
$$

Assume also that $u$ satisfies the damping conditions

$$
\int_{|y|=R}\left|D_{k}^{j} u(y)\right|^{2} d \mathscr{H}^{n-1}(y)=\left\{\begin{array}{ll}
o\left(R^{2-j}\right) & \text { if } j \text { is even, } \\
o\left(R^{3-j}\right) & \text { if } j \text { is odd },
\end{array} \text { as } R \rightarrow \infty,\right.
$$


for all $j \in\{0,1, \ldots, 2 N-1\}$. Then for every $x \in \Omega$ we have

$$
\begin{aligned}
u(x)=\int_{\partial \Omega} \sum_{j=1}^{N} & \left\{\left(D_{k} \Phi_{k}^{(j)}\right)(x-y) \odot v(y) \odot\left(\Delta+k^{2}\right)^{j-1} u(y)\right. \\
& \left.+\Phi_{k}^{(j)}(x-y) v(y) \odot D_{k}\left(\Delta+k^{2}\right)^{j-1} u(y)\right\} d \mathscr{H}^{n-1}(y) .
\end{aligned}
$$

Consequently, for every $x \in \Omega$ we have

$$
\begin{aligned}
u(x) & =\sum_{j=1}^{N} \int_{\partial \Omega}\left\{\left(D \Phi_{k}^{(j)}\right)(x-y) \odot v(y) \odot\left(\Delta+k^{2}\right)^{j-1} u(y)\right. \\
& \left.+\Phi_{k}^{(j)}(x-y) v(y) \odot D\left(\Delta+k^{2}\right)^{j-1} u(y)\right\} d \mathscr{H}^{n-1}(y) .
\end{aligned}
$$

Proof. Since $D_{k}^{2}=-\left(\Delta+k^{2}\right)$, we have that $u$ satisfies $D_{k}^{2 N} u=0$ in $\Omega$. Also, from (8.3) and (7.22) (used with $N$ replaced by $2 N$ ) it follows that

$$
i k w_{N}(y)+\widehat{y} \odot D w_{N}(y)=Q_{2 N}\left(y, D_{k}\right) u \quad \text { for every } y \in \Omega \text {. }
$$

Returning with (8.7) to (8.2) we obtain that $u$ satisfies (7.37). Since $u$ also satisfies (7.24) (keeping in mind (8.4)), we invoke Theorem 7.9 to conclude that $u$ radiates in $\Omega$. Hence, Theorem 7.11 applies and gives the integral representation formula

$$
u(x)=-\int_{\partial \Omega} \sum_{j=0}^{2 N-1}\left\{\Theta_{k}^{(j+1)}(x-y) \odot v(y) \odot D_{k}^{j} u(y)\right\} d \mathscr{H}^{n-1}(y) \quad \text { for every } x \in \Omega .
$$

Next, in the sum in the right hand-side of (8.8), we combine the terms corresponding 
to $j=2 \ell-2$ and $j=2 \ell-1$ for each $\ell \in\{1, \ldots, N\}$ as

$$
u(x)=\sum_{\ell=1}^{N} \int_{\partial \Omega} A_{\ell}(x, y) d \mathscr{H}^{n-1}(y),
$$

where

$$
\begin{aligned}
A_{\ell}(x, y):= & -\Theta_{k}^{(2 \ell-1)}(x-y) \odot v(y) \odot\left(D_{k}^{2 \ell-2} u\right)(y) \\
& -\Theta_{k}^{(2 \ell)}(x-y) \odot v(y) \odot\left(D_{k}^{2 \ell-1} u\right)(y)
\end{aligned}
$$

for each $y \in \partial \Omega$ and each $\ell \in\{1, \ldots, N\}$. In addition, (6.1) and (2.27) further gives

$$
\begin{array}{r}
A_{\ell}(x, y)=\left(D_{k} \Phi_{k}^{(\ell)}\right)(x-y) \odot v(y) \odot\left(\Delta+k^{2}\right)^{\ell-1} u(y) \\
+\Phi_{k}^{(\ell)}(x-y) v(y) \odot D_{k}\left(\Delta+k^{2}\right)^{\ell-1} u(y)
\end{array}
$$

for each $y \in \partial \Omega$ and each $\ell \in\{1, \ldots, N\}$. Now (8.5) follows by combining (8.9) and (8.11).

If we further use (2.24) in the right hand-side of (8.11) we see that

$$
\begin{aligned}
A_{\ell}(x, y)= & \left(D \Phi_{k}^{(\ell)}\right)(x-y) \odot v(y) \odot\left(\Delta+k^{2}\right)^{\ell-1} u(y) \\
& +k \Phi_{k}^{(\ell)}(x-y) e_{n+1} \odot v(y) \odot\left(\Delta+k^{2}\right)^{\ell-1} u(y) \\
& +\Phi_{k}^{(\ell)}(x-y) \odot v(y) \odot D\left(\Delta+k^{2}\right)^{\ell-1} u(y) \\
& +k \Phi_{k}^{(\ell)}(x-y) v(y) \odot e_{n+1} \odot\left(\Delta+k^{2}\right)^{\ell-1} u(y)
\end{aligned}
$$

for each $y \in \partial \Omega$ and each $\ell \in\{1, \ldots, N\}$. Since as a consequence of (2.2) and $v(y) \in \mathbb{R}^{n}$ 
we have

$$
e_{n+1} \odot v=-v \odot e_{n+1}
$$

formula (8.12) becomes

$$
\begin{aligned}
A_{\ell}(x, y)= & \left(D \Phi_{k}^{(\ell)}\right)(x-y) \odot v(y) \odot\left(\Delta+k^{2}\right)^{\ell-1} u(y) \\
& +\Phi_{k}^{(\ell)}(x-y) \odot v(y) \odot D\left(\Delta+k^{2}\right)^{\ell-1} u(y),
\end{aligned}
$$

for each $y \in \partial \Omega$ and every $\ell \in\{1, \ldots, N\}$. Now the integral representation formula (8.6) follows by combining (8.14) and (8.9).

\section{Remark 8.2.}

(1) A version of Theorem 8.1 in which condition (8.2) is replaced by

$$
\int_{|y|=R}\left|\sum_{j=0}^{N-1} \frac{|y|^{j}}{j !(2 i k)^{j}}\left[i k\left(\Delta+k^{2}\right)^{j} u(y)+\widehat{y} \odot D\left(\Delta+k^{2}\right)^{j} u(y)\right]\right|^{2} d \mathscr{H}^{n-1}(y)=o(1)
$$

as $R \rightarrow \infty$ remains valid. Indeed, this is a consequence of Definition 7.6, (7.20), and Theorem 7.9.

(2) In the case $N=1$, in view of part (1) above we see that Theorem 7.11 states that if $u \in \mathscr{C}^{2}\left(\bar{\Omega}, C \ell_{m}\right)$ is a null-solution of the Helmholtz operator $\Delta+k^{2}$ in $\Omega$ and satisfies (7.28) then, for every $x \in \Omega$, u has the integral representation formula

$$
\begin{aligned}
u(x)= & \int_{\partial \Omega}\left\{\left(D \Phi_{k}\right)(x-y) \odot v(y) \odot u(y)+\Phi_{k}(x-y) v(y) \odot D u(y)\right\} d \mathscr{H}^{n-1}(y) . \\
= & \int_{\partial \Omega}\left(D_{k} \Phi_{k}\right)(x-y) \odot v(y) \odot u(y) d \mathscr{H}^{n-1}(y) \\
& +\int_{\partial \Omega} \Phi_{k}(x-y) \odot v(y) \odot D_{k} u(y) d \mathscr{H}^{n-1}(y)
\end{aligned}
$$


(recall (8.13) for the second equality). This matches the integral representation formula in [9, Theorem 1.1, part (B3)]. 


\section{A Liouville type theorem for iterated perturbed Dirac operators}

The classical Liouville Theorem for harmonic functions states that any $u \in \mathscr{C}^{\infty}\left(\mathbb{R}^{n}\right)$ with $\Delta u=0$ in $\mathbb{R}^{n}$ which is bounded is necessarily a constant. The condition that $u$ is bounded may be interpreted as "the correct" radiation condition for the Laplacian. If we replace $\Delta$ with $\Delta+k^{2}$, for some $k \in(0, \infty)$, we have that if $u \in \mathscr{C}^{\infty}\left(\mathbb{R}^{n}, C \ell_{m}\right)$ is a null solution of the Helmholtz operator in $\mathbb{R}^{n}$, i.e., $\left(\Delta+k^{2}\right) u=0$ in $\mathbb{R}^{n}$, and $u$ satisfies the Sommerfeld radiation condition (1.1), then $u=0$ in $\mathbb{R}^{n}$ (cf. [9, Lemma 3.8]). Our next result is a higher-order analogue of this phenomenon. Recall Definition 7.6.

Theorem 9.1. Let $k \in(0, \infty)$ and $N \in \mathbb{N}$. Suppose $u \in \mathscr{C}^{N}\left(\mathbb{R}^{n}, C \ell_{m}\right)$ is such that $D_{k}^{N} u=0$ in $\mathbb{R}^{n}$ and $u$ is radiating in $\mathbb{R}^{n}$. Then necessarily $u=0$ in $\mathbb{R}^{n}$.

Proof. Fix $x_{0} \in \mathbb{R}^{n}$ arbitrary along with some $\varepsilon \in(0,1)$ and consider the domain $\Omega_{\varepsilon}:=\mathbb{R}^{n} \backslash \overline{B\left(x_{0}, \varepsilon\right)}$. Then Theorem 7.11 applies and gives that

$$
u(x)=\sum_{j=0}^{N-1} \int_{\left|y-x_{0}\right|=\varepsilon} \Theta_{k}^{(j+1)}(x-y) \odot\left(\frac{y-x_{0}}{\varepsilon}\right) \odot D_{k}^{j} u(y) d \mathscr{H}^{n-1}(y)
$$

for every $x \in \Omega_{\varepsilon}$.

Now fix $x \in \Omega_{\varepsilon}$, set $r:=\left|x-x_{0}\right|>0$ and impose the additional restriction $\varepsilon<r / 2$. Then

$$
\left\{\begin{array}{l}
|y-x| \geq\left|x_{0}-x\right|-\left|y-x_{0}\right|=r-r / 2=r / 2 \\
|x-y| \leq\left|x-x_{0}\right|+\left|y-x_{0}\right|=r+r / 2=3 r / 2
\end{array} \quad \text { for all } y \in \partial B\left(x_{0}, \varepsilon\right) .\right.
$$


Making use of (9.1), (2.7), (2.17) (which applies given (6.1)), and (9.2) we estimate

$$
|u(x)| \leq\left(\sum_{|\alpha| \leq N-1}\left\|\partial^{\alpha} u\right\|_{L^{\infty}(\overline{B(0,1))})}\right)\left(\sum_{j=0}^{N-1}\left\|\Theta_{k}^{(j+1)}\right\|_{L^{\infty}(\overline{B(0,3 r / 2)} \backslash B(0, r))}\right) \varepsilon^{n-1} .
$$

Letting now $\varepsilon \rightarrow 0^{+}$in (9.3) yields $u(x)=0$ as wanted. Hence $u=0$ in $\Omega_{\varepsilon}$. Since $\varepsilon$ is arbitrary in $(0,1)$ we obtain $u=0$ in $\mathbb{R}^{n} \backslash\left\{x_{0}\right\}$, which in turn implies $u=0$ in $\mathbb{R}^{n}$ given that $x_{0} \in \mathbb{R}^{n}$ was arbitrary. 


\section{Appendix}

\section{A Properties of Hankel functions}

Recall that $H_{\lambda}^{(1)}(\cdot)$ denotes the Hankel function of the first kind with index $\lambda \in \mathbb{R}$. Some of its basic properties are reviewed next (see [18]).

Lemma A.1. Let $\lambda \in \mathbb{R}$ and suppose $r>0$. Also, fix an arbitrary $N \in \mathbb{N}$. Then the following properties of Hankel functions of the first kind hold:

$$
\begin{aligned}
H_{-\lambda}^{(1)}(r) & =e^{i \pi \lambda} H_{\lambda}^{(1)}(r), \\
\frac{d}{d r}\left[r^{\lambda} H_{\lambda}^{(1)}(r)\right] & =r^{\lambda} H_{\lambda-1}^{(1)}(r), \\
\frac{d}{d r}\left[r^{-\lambda} H_{\lambda}^{(1)}(r)\right] & =-r^{-\lambda} H_{\lambda+1}^{(1)}(r), \\
\frac{d}{d r} H_{\lambda}^{(1)}(r) & =H_{\lambda-1}^{(1)}(r)-\frac{\lambda}{r} H_{\lambda}^{(1)}(r), \\
\frac{d}{d r} H_{\lambda}^{(1)}(r) & =-H_{\lambda+1}^{(1)}(r)+\frac{\lambda}{r} H_{\lambda}^{(1)}(r), \\
\frac{2 \lambda}{r} H_{\lambda}^{(1)}(r) & =H_{\lambda-1}^{(1)}(r)+H_{\lambda+1}^{(1)}(r), \\
\frac{d}{d r} H_{\lambda}^{(1)}(r) & =\frac{1}{2}\left[H_{\lambda-1}^{(1)}(r)-H_{\lambda+1}^{(1)}(r)\right], \\
\left(\frac{d}{d r}\right)^{N} H_{\lambda}^{(1)}(r) & =\frac{1}{2^{N}} \sum_{j=0}^{N}(-1)^{j}\left(\begin{array}{c}
N \\
j
\end{array}\right) H_{\lambda-N+2 j}^{(1)}(r), \\
H_{\lambda}^{(1)}(r) & =\left(\frac{2}{\pi r}\right)^{1 / 2} e^{i(r-\lambda \pi / 2-\pi / 4)}+O\left(r^{-3 / 2}\right) \quad \text { as } r \rightarrow \infty .
\end{aligned}
$$


Lemma A.2. Let $\lambda \in \mathbb{R}$. Then the following limits hold:

$$
\begin{gathered}
\lim _{r \rightarrow 0^{+}} \frac{\left|H_{\lambda}^{(1)}(r)\right|}{\pi}|\ln (r)| \\
\lim _{r \rightarrow 0^{+}} \frac{\left|H_{\lambda}^{(1)}(r)\right|}{\frac{2^{|\lambda|} \Gamma(|\lambda|)}{\pi} r^{-|\lambda|}}=1 \quad \text { if } \quad \lambda=0,
\end{gathered}
$$

Proof. Corresponding to $\lambda \in[0, \infty)$, from $[18,10.7 .2 \& 10.7 .7]$ we have

$$
\lim _{r \rightarrow 0^{+}} \frac{H_{0}^{(1)}(r)}{\frac{2 i}{\pi} \ln (r)}=1 \quad \text { and } \quad \lim _{r \rightarrow 0^{+}} \frac{H_{\lambda}^{(1)}(r)}{\frac{2 \lambda}{i \pi} \Gamma(\lambda) r^{-\lambda}}=1
$$

This proves (A.10). If $\lambda \in(-\infty, 0)$, from (A.1) we know $H_{\lambda}^{(1)}(r)=e^{-i \pi \lambda} H_{-\lambda}^{(1)}(r)$ for $r>0$. On the other hand, the second limit in (A.12) gives

$$
\lim _{r \rightarrow 0^{+}} \frac{e^{-i \pi \lambda} H_{-\lambda}^{(1)}(r)}{\frac{2^{-\lambda} e^{-i \pi \lambda}}{i \pi} \Gamma(-\lambda) r^{\lambda}}=1
$$

Consequently,

$$
\lim _{r \rightarrow 0^{+}} \frac{H_{\lambda}^{(1)}(r)}{\frac{2^{-\lambda} e^{-i \pi \lambda}}{i \pi} \Gamma(-\lambda) r^{\lambda}}=1 \quad \forall \lambda \in(-\infty, 0) .
$$

Now (A.11) is immediate from (A.12) and (A.14).

Additional asymptotic expansions which play an important role in our analysis are singled out in the next lemma.

Lemma A.3. Let $\lambda \in \mathbb{R}, N \in \mathbb{N}$, and suppose $r>0$. Then the following asymptotic 
expansions of Hankel functions of the first kind and their derivatives hold:

$$
\begin{aligned}
H_{\lambda}^{(1)}(r) & =O\left(r^{-1 / 2}\right) \quad \text { as } r \rightarrow \infty, \\
\frac{d}{d r} H_{\lambda}^{(1)}(r) & =H_{\lambda-1}^{(1)}(r)+O\left(r^{-3 / 2}\right) \quad \text { as } r \rightarrow \infty, \\
\left(\frac{d}{d r}\right)^{N} H_{\lambda}^{(1)}(r) & =O\left(r^{-1 / 2}\right) \quad \text { as } r \rightarrow \infty, \\
\left(\frac{d}{d r}\right)^{N} H_{\lambda}^{(1)}(r) & =H_{\lambda-N}^{(1)}(r)+O\left(r^{-3 / 2}\right) \quad \text { as } r \rightarrow \infty .
\end{aligned}
$$

Proof. Property (A.15) follows directly from (A.9), while (A.15) combined with (A.4) yields (A.16). Also, (A.15) together with (A.8) gives (A.17). We are left with proving (A.18). First, we claim that for each $N \in \mathbb{N}$,

$$
\left(\frac{d}{d r}\right)^{N} H_{\lambda}^{(1)}(r)=\sum_{j=0}^{N} C_{N, j}^{\lambda} \frac{1}{r^{N-j}} H_{\lambda-j}^{(1)}(r)
$$

where $C_{N, j}^{\lambda} \in \mathbb{C}$ are constants depending only on $N$, $j$, and $\lambda$, defined as follows. Corresponding to $N=1$ we take

$$
C_{1,0}^{\lambda}:=-\lambda \text { and } C_{1,1}^{\lambda}:=1 \text {, }
$$

then for each $N \in \mathbb{N}$ we recursively define

$$
C_{N+1, j}^{\lambda}:= \begin{cases}1 & \text { if } j=N+1, \\ C_{N, j}^{\lambda}(2 j-N-\lambda)+C_{N, j-1}^{\lambda} & \text { if } 1 \leq j \leq N, \\ C_{N, 0}^{\lambda}(-N-\lambda) & \text { if } j=0 .\end{cases}
$$

We shall now prove that formula (A.19) holds for the choice of coefficients as in 
(A.20)-(A.21) via an induction argument over $N$. That the corresponding statement for $N=1$ is true is seen directly from (A.4) and (A.20). Suppose next that (A.19) holds for some $N \in \mathbb{N}$. By differentiating (A.19) one more time and using (A.4) we arrive at

$$
\begin{aligned}
\left(\frac{d}{d r}\right)^{N+1} H_{\lambda}^{(1)}(r) & =\sum_{j=0}^{N} C_{N, j}^{\lambda} \frac{j-N}{r^{N+1-j}} H_{\lambda-j}^{(1)}(r)+\sum_{j=0}^{N} C_{N, j}^{\lambda} \frac{1}{r^{N-j}}\left[H_{\lambda-j-1}^{(1)}(r)-\frac{\lambda-j}{r} H_{\lambda-j}^{(1)}(r)\right] \\
& =\sum_{j=0}^{N} C_{N, j}^{\lambda} \frac{2 j-N-\lambda}{r^{N+1-j}} H_{\lambda-j}^{(1)}(r)+\sum_{j=1}^{N+1} C_{N, j-1}^{\lambda} \frac{1}{r^{N+1-j}} H_{\lambda-j}^{(1)}(r) \\
& =\sum_{j=0}^{N+1} C_{N+1, j}^{\lambda} \frac{1}{r^{N+1-j}} H_{\lambda-j}^{(1)}(r),
\end{aligned}
$$

where the last step uses the recurrence formula (A.21). This completes the proof of (A.19).

Moving on, observe that formula (A.19) may be written as

$$
\left(\frac{d}{d r}\right)^{N} H_{\lambda}^{(1)}(r)=H_{\lambda-N}^{(1)}(r)+\sum_{j=0}^{N-1} C_{N, j}^{\lambda} \frac{1}{r^{N-j}} H_{\lambda-j}^{(1)}(r) .
$$

When used in concert with (A.15), this now readily yields (A.18).

A combination of LemmaA.3 and the Chain Rule yields asymptotic expansions for derivatives with respect to $x$ of $H_{\lambda}^{(1)}(k|x|)$. These are collected in the next proposition. The reader is reminded that for each $x \in \mathbb{R}^{n} \backslash\{0\}$ we abbreviate $\widehat{x}:=x /|x|$.

Proposition A.4. Let $\lambda \in \mathbb{R}, k \in(0, \infty)$, and fix a multi-index $\beta \in \mathbb{N}_{0}^{n}$ with $|\beta|>0$. Then 
the following asymptotic expansions hold:

$$
\begin{aligned}
& \partial^{\beta}\left[H_{\lambda}^{(1)}(k|x|)\right]=H_{\lambda-|\beta|}^{(1)}(k|x|)(k \widehat{x})^{\beta}+O\left(|x|^{-3 / 2}\right) \text { as }|x| \rightarrow \infty, \\
& \partial^{\beta}\left[H_{\lambda}^{(1)}(k|x|)\right]=\left(\left(\frac{2}{\pi k|x|}\right)^{1 / 2} e^{i(k|x|-\lambda \pi / 2-\pi / 4)}\right)(i k \widehat{x})^{\beta}+O\left(|x|^{-3 / 2}\right) \text { as }|x| \rightarrow \infty .
\end{aligned}
$$

Proof. Fix a multi-index $\beta=\left(\beta_{1}, \ldots, \beta_{n}\right) \in \mathbb{N}_{0}^{n}$ of positive length. For starters observe that repeated applications of the Chain Rule give that, for $x \in \mathbb{R}^{n} \backslash\{0\}$,

$$
\begin{aligned}
\partial^{\beta}\left[H_{\lambda}^{(1)}(k|x|)\right]= & k^{|\beta|}\left(\left(\frac{d}{d r}\right)^{|\beta|} H_{\lambda}^{(1)}\right)(k|x|)\left(\partial_{1}(|x|)\right)^{\beta_{1}} \cdot\left(\partial_{2}(|x|)\right)^{\beta_{2}} \cdots\left(\partial_{n}(|x|)\right)^{\beta_{n}} \\
& +\sum_{\ell=1}^{|\beta|-1} k^{\ell}\left(\left(\frac{d}{d r}\right)^{\ell} H_{\lambda}^{(1)}\right)(k|x|) \sum_{\alpha_{1}+\cdots+\alpha_{\ell}=\beta} C_{\alpha_{1}, \ldots, \alpha_{\ell}} \partial^{\alpha_{1}}(|x|) \cdots \partial^{\alpha_{\ell}}(|x|),
\end{aligned}
$$

with the convention that the sum over $\ell$ is void if $|\beta|=1$. Above, $C_{\alpha_{1}, \ldots, \alpha_{\ell}}$ are constants depending only on the multi-indices $\alpha_{1}, \ldots, \alpha_{\ell} \in \mathbb{N}_{0}^{n}$. Since $\partial_{j}(|x|)=\frac{x_{j}}{|x|}=\widehat{x}_{j}$ for each $j \in\{1, \ldots, n\}$, we may write (A.26) as

$$
\begin{aligned}
\partial^{\beta}\left[H_{\lambda}^{(1)}(k|x|)\right]= & \left(\left(\frac{d}{d r}\right)^{|\beta|} H_{\lambda}^{(1)}\right)(k|x|)(k \widehat{x})^{\beta} \\
& +\sum_{\ell=1}^{|\beta|-1} k^{\ell}\left(\left(\frac{d}{d r}\right)^{\ell} H_{\lambda}^{(1)}\right)(k|x|) \sum_{\alpha_{1}+\cdots+\alpha_{\ell}=\beta} C_{\alpha_{1}, \ldots, \alpha_{\ell}} \partial^{\alpha_{1}}(|x|) \cdots \partial^{\alpha_{\ell}}(|x|),
\end{aligned}
$$

again, with the convention that the sum over $\ell$ is void if $|\beta|=1$. Invoking (A.18), we further transform

$$
\begin{aligned}
\left(\left(\frac{d}{d r}\right)^{|\beta|} H_{\lambda}^{(1)}\right)(k|x|)(k \widehat{x})^{\beta} & =\left(H_{\lambda-|\beta|}^{(1)}(k|x|)+O\left(|x|^{-3 / 2}\right)\right)(k \widehat{x})^{\beta} \\
& =H_{\lambda-|\beta|}^{(1)}(k|x|)(k \widehat{x})^{\beta}+O\left(|x|^{-3 / 2}\right) \text { as }|x| \rightarrow \infty .
\end{aligned}
$$


On the other hand, note that $\partial^{\gamma}(|x|)=O\left(|x|^{1-|\gamma|}\right)$ as $|x| \rightarrow \infty$, for any $\gamma \in \mathbb{N}_{0}^{n}$. On account of this observation we then conclude that, if $|\beta|>1$, for each $\ell \in\{1, \ldots,|\beta|-1\}$ we have

$$
\sum_{\alpha_{1}+\cdots+\alpha_{\ell}=\beta} C_{\alpha_{1}, \ldots, \alpha_{\ell}} \partial^{\alpha_{1}}(|x|) \cdots \partial^{\alpha_{\ell}}(|x|)=O\left(|x|^{\ell-|\beta|}\right)=O\left(|x|^{-1}\right) \quad \text { as }|x| \rightarrow \infty .
$$

Now (A.24) follows by combining (A.27), (A.28), (A.29) and (A.17). Finally, (A.25) is a direct consequence of (A.24) and (A.9).

\section{B A version of the Divergence Theorem}

We shall need a version of the Divergence Theorem which is a particular case of the sharp version from [13] proved there in a much more general setting.

Theorem B.1 (Divergence Theorem; [13]). Let $\Omega \subseteq \mathbb{R}^{n}$ be a Lipschitz domain with compact boundary and outward unit normal $v=\left(v_{j}\right)_{1 \leq j \leq n}$. Consider a vector field

$$
\vec{F}=\left(F_{j}\right)_{1 \leq j \leq n} \in\left[\mathscr{C}^{0}\left(\bar{\Omega}, C \ell_{m}\right)\right]^{n}
$$

with the property that div $\vec{F}$, taken in the sense of distributions in $\mathcal{D}^{\prime}\left(\Omega, C \ell_{m}\right)$, satisfies $\operatorname{div} \vec{F} \in L^{1}\left(\Omega, C \ell_{m}\right)$. If $\Omega$ is unbounded also assume

$$
\lim _{R \rightarrow \infty} \int_{|x|=R} \sum_{j=1}^{n} \frac{x_{j}}{|x|} F_{j}(x) d \mathscr{H}^{n-1}(x)=0
$$

Then,

$$
\int_{\Omega} \operatorname{div} \vec{F} d \mathscr{L}^{n}=\left.\int_{\partial \Omega} \sum_{j=1}^{n} v_{j} F_{j}\right|_{\partial \Omega} d \mathscr{H}^{n-1}
$$




\section{References}

[1] M. Abramowitz and I.A. Stegun, Handbook of Mathematical Functions, Dover, New York, 1972.

[2] W. W. Bell, Special Functions for Scientists and Engineers, Dover, New York, 2004.

[3] F. Brackx, R. Delanghe and F. Sommen, Clifford Analysis, Research Notes in Mathematics, Vol. 76, Pitman, Boston, MA, 1982.

[4] B. C. Carlson, Special Functions of Applied Mathematics, Academic Press, New York, San Francisco, London, 1997.

[5] D. Colton and R. Kress, Integral Equation Methods in Scattering Theory, Wiley Interscience Publications, New York, 1983.

[6] D. Colton and R. Kress, Inverse Acoustic and Electromagnetic Scattering Theory, Springer-Verlag, Applied Math. Series, No. 93, 1992.

[7] J. Gilbert and M.A. Murray, Clifford Algebras and Dirac Operators in Harmonic Analysis, Cambridge Studies in Advanced Mathematics, 1991.

[8] B. Jawerth and M. Mitrea, Higher dimensional scattering theory on $C^{1}$ and Lipschitz domains, Amer. J. of Math. 117, 929-963 (1995).

[9] E. Marmolejo-Olea, D. Mitrea, I. Mitrea, and M. Mitrea, Radiation Conditions and Integral Representations for Clifford Algebra-Valued Null-Solutions of the Helmholtz Operator, preprint, (2015).

[10] E. Marmolejo-Olea and S. Pérez-Esteva, Far-field patterns of solutions of the perturbed Dirac equation, Math. Meth. Appl. Sci. 36, 1376-1387, (2013).

[11] A. McIntosh and M. Mitrea, Clifford algebras and Maxwell's equations in Lipschitz domains, Math. Meth. Appl. Sci. 22, 1599-1620, (1999).

[12] D. Mitrea, Distributions, Partial Differential Equations, and Harmonic Analysis, Springer, New York, 2013.

[13] D. Mitrea, I. Mitrea and M. Mitrea, A sharp Divergence Theorem with nontangential pointwise traces, preprint, (2014).

[14] M. Mitrea, Clifford Wavelets, Singular Integrals, and Hardy Spaces, Lecture Notes in Mathematics, No. 1575, Springer-Verlag, Berlin, Heidelberg, New York, 1994.

[15] M. Mitrea, Generalized Dirac operators on non-smooth manifolds and Maxwell's equations, Journal of Fourier Analysis and Applications, Vol. 7, No. 3, 207-256 (2001). 
[16] C. Müller, Über die Beugung elektromagnetischer Schwingungen an endlichen homogenen Körpern, Math. Ann. 123, 345-378 (1951).

[17] C. Müller, Foundations of the Mathematical Theory of Electromagnetic Waves, Springer-Verlag, Berlin, Heidelberg, New York, 1969.

[18] F. Olver, NIST Digital Library of Mathematical Functions, Chapter 10, http://dlmf.nist.gov/10, [edited by] Frank W.J. Olver and L. C. Maximon, New York: Cambridge University Press: NIST, 2010.

[19] F. Rellich, Uber das asymptotische Verhalten der Losungen von $\Delta u+\lambda u=0$ in unendlichen Gebieten, Jber. Deutsch. Math. Verein. 53, 57-65 (1943).

[20] S. Schot, Eighty years of Sommerfeld's radiation condition, Historia Mathematica, 19, 393-396 (1992).

[21] A. Sommerfeld, Die Greensche Function der Schwingungsgleichgung, Jahresbeircht der Deutschen Mathematiker-Vereinigung 21, 309-353 (1912). Reprinted in Gesammelte Schiriften, vol. 1, 272-316.

[22] I. V. Subeika, The exterior boundary value problem for an operator which can be split into Helmholtz operators, Differential Equations 4, 859-864 (1968).

[23] B. R. Vainberg, Principles of radiation, limiting absorbtion and limiting amplitude in the general theory of partial differential equations, Russian Math. Surveys 21, No. 3, 115-193 (1966).

[24] I. N. Vekua, Metaharmonic functions, Trudy Tbilisskogo Matematicheskogo Instituta 12, 105-175 (1943).

[25] H. Weyl, Kapazität von Strahlungsfeldern, Math. Zeit. 55, 187-198 (1952).

[26] H. Weyl, Die natürlichen Randwertaufgaben im Aussenraum für Strahlungsfeldern beliebiger Dimensionen und beliebiger Ranges, Math. Zeit. 56, 105-119 (1952).

[27] K. J. Witsch, The Exterior Dirichlet Problem for a Class of Fourth Order Elliptic Equations, Journal of Mathematical Analysis and Applications 49, 734-747 (1975).

[28] K. J. Witsch, Radiation Conditions and the Exterior Dirichlet Problem for a Class of Higher Order Elliptic Equations, Journal of Mathematical Analysis and Applications 54, 820-839 (1976). 
Nicholas Okamoto was born in December of 1978 on the Big Island of Hawaii. After receiving his high school diploma from H. P. Baldwin High School in 1996, he offered a period of service at a vocational school on the Tarawa atoll of Kiribati. Returning to Hawaii a year later, he went on to earn an Associate in Arts degree in Liberal Arts from the University of Hawaii Maui College in 1999, followed by a Bachelor of Arts degree in Mathematics and Physics from the University of Hawaii at Hilo in 2003. He spent the next two years as a math specialist at the Kyoiku Juku Tutorial Institute on the island of Oahu.

In 2005 Nick was awarded a Math for America Newton Fellowship involving a five-year commitment in New York City. During the first year of his Newton Fellowship he earned a Master of Arts degree in Mathematics Education from Teachers College Columbia University. For the remaining four years of his Newton Fellowship he was a math teacher at MS 54 Booker T. Washington Middle School.

Upon completion of his Newton Fellowship Program in 2010, Nick went on to become a graduate student and teaching assistant at the University of Missouri. While at Mizzou he completed a Master of Science degree in Applied Mathematics in 2012, and anticipates completing a Doctor of Philosophy degree in Mathematics in 2017.

Nick was recently offered a tenure track faculty position at the University of Hawaii Maui College to begin in the fall of 2017. He eagerly accepted this position, and is very excited to be moving back to his home state. 